\title{
LINE-WISE POWER BALANCE EQUATIONS AND APPLICATIONS
}

by

\author{
Amr Adel Fathy Mohamed \\ Master of Science in Engineering, Cairo University, Egypt, 2012 \\ Bachelor of Engineering, Cairo University, Egypt, 2008
}

\author{
A dissertation \\ presented to Ryerson University \\ in partial fulfillment of the \\ requirements for the degree of \\ Doctor of Philosophy \\ in the Program of \\ Electrical and Computer Engineering
}

Toronto, Ontario, Canada, 2019

(C) Amr Mohamed, 2019 


\section{Author'S DEClaration}

I hereby declare that I am the sole author of this dissertation. This is a true copy of the dissertation, including any required final revisions, as accepted by my examiners.

I authorize Ryerson University to lend this dissertation to other institutions or individuals for the purpose of scholarly research.

I further authorize Ryerson University to reproduce this dissertation by photocopying or by other means, in total or in part, at the request of other institutions or individuals for the purpose of scholarly research.

I understand that my dissertation may be made electronically available to the public. 


\title{
Abstract
}

\section{LINE-WISE POWER BALANCE EQUATIONS AND APPLICATIONS}

\author{
Amr Adel Fathy Mohamed \\ Doctor of Philosophy \\ Electrical and Computer Engineering \\ Ryerson University, 2019
}

Optimal power flow (OPF) refers to optimize power systems considering a chosen objective subject to a set of constraints. Existing OPF formulations used to settle electricity markets include a set of bus-wise power balance equations (PBE) that is comprised exclusively of high order terms which have sinusoidal components. Accordingly, such OPF formulations remain nonlinear and nonconvex optimization problems. Even though commercial OPF solvers are robust and efficient, they still cannot guarantee a global optimum. The US Federal Energy Regulatory Commission estimates that the best commercial OPF solvers are off by $10 \%$, amounting to an annual loss of US $\$ 400$ billion worldwide. For these motivating reasons, OPF remains a major research focus and forms the topic of this thesis.

This thesis aims to: (1) develop new sets of PBE with lower order terms and lesser numbers of sinusoidal terms yielding better solution space, (2) build new OPF formulations using this new set of PBE, and (3) incorporate voltage stability constraints into the developed OPF formulations. The genesis of the new set of PBE stems from: (1) the fact that power of a constant impedance load is proportional to the square of voltage magnitude, and, (2) power flow in branches can be expressed in terms of square of voltage magnitude. Accordingly, a set of line-wise PBE is developed, both in polar and rectangular forms and are solved Newton-Raphson technique. Tests show that the 
proposed line-wise power flow (LWPF) algorithms are accurate, provide monotonic convergence, and scale well for large systems. The algorithms are faster comparing to classical bus-wise power flow methods. Further, the ability to directly identify the set of critical lines that are the most susceptible to Voltage collapse.

A novel line-wise optimal power flow (LWOPF) formulation is developed based on polar LWPF and solved using successive linear programming technique. Tests show that LWOPF consistently yields a better solution than that computed using bus-wise OPF, namely in half the time. LWOPF is extended to include voltage stability constraints and implemented using both linear and nonlinear optimization techniques. It demonstrates improved performance in achieving lower cost optimal solutions with better voltage-stable states. 


\section{ACKNOWLEDGMENTS}

First and foremost, it is by the grace of Almighty Allah (my God), the most merciful and most gracious, that this thesis has been completed. I am sincerely appreciative to His limitless blessings in every step of my life, encircling me with all the supportive and affectionate people throughout my journey.

My sincere gratitude is to my advisor, Professor Bala Venkatesh, for his guidance, enthusiastic encouragement and honest advice. His willingness to give his time so generously has been very much appreciated. I am forever grateful for his influence on reshaping my academic personality. His attitude and the knowledge he imparts have an impact, challenging us to move beyond the status quo.

I would like to express my special thanks to Dr. Amirnaser Yazdani, Dr. Alagan Anpalagan, and Dr. David Xu for supervising my candidacy examination. Their comments and suggestions have been very helpful for me to conduct this research work.

I am very grateful to Dr. Mohamed Awadallah, Dr. Walmir Freitas, and Dr. Chandrabhanu Opathella for their invaluable comments and suggestions. Additionally, I would like to thank Mr. Ayman Elkasrawy, Dr. Carlos Sabillón and all present and past colleagues in the Center for Urban Energy (CUE) at Ryerson University for their support and encouragement.

I would also like to acknowledge the support that I received from the faculty and staff in the Department of Electrical and Computer Engineering at Ryerson University in Toronto.

Just as importantly, no words can describe the unprecedented support that I have received, and continue to get from my family. I am sincerely grateful to my parents, my wife, my siblings, my children, and my entire family for their patience, endless support, and encouragement. 


\section{DEDICATION}

To my father, mother, wife, siblings, and lovely children Ans, Arwa and Sarah 


\section{Table of contents}

Author's Declaration ............................................................................................................................ ii

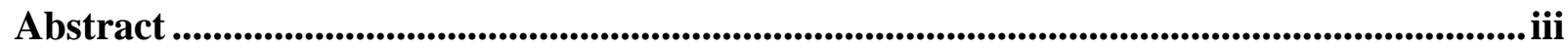

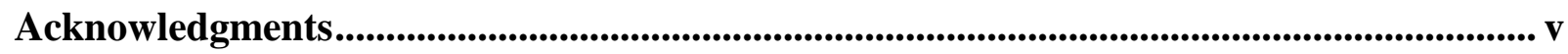

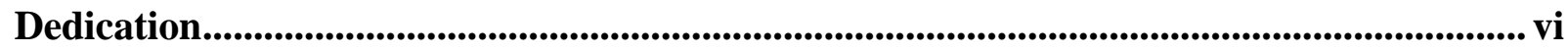

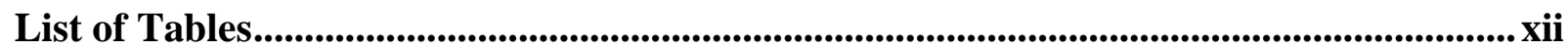

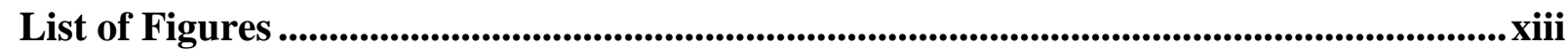

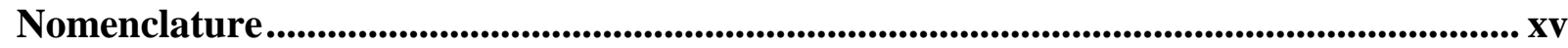

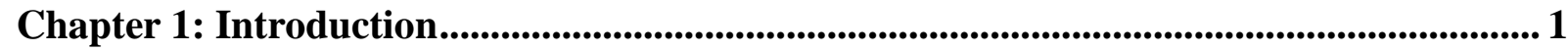

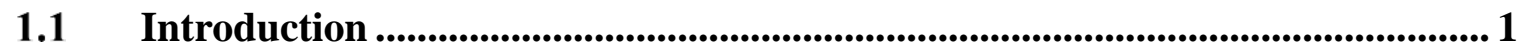

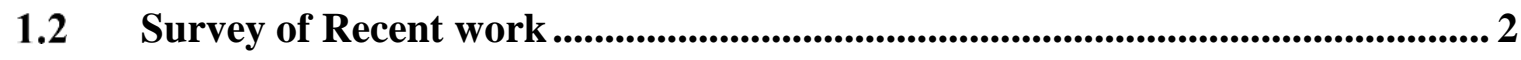

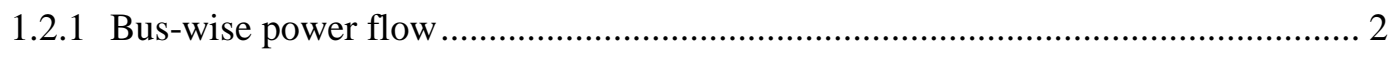

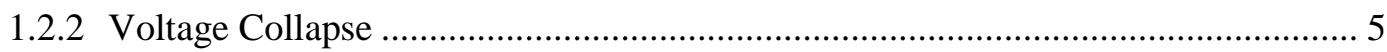

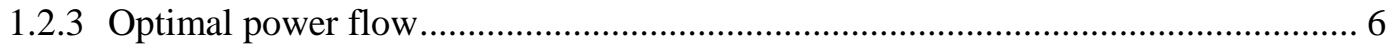

1.2.4 Voltage stability constrained optimal power flow......................................... 10

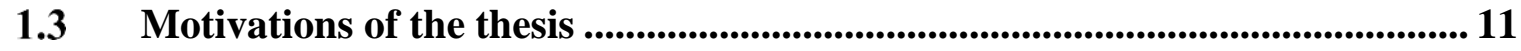

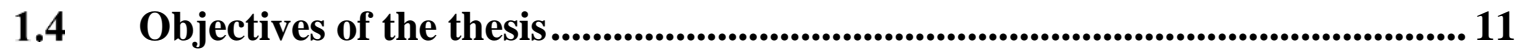

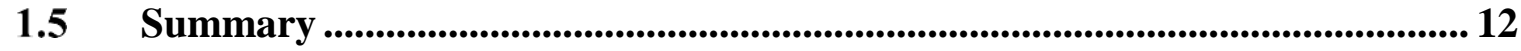

Chapter 2: Polar Line-Wise Power Flow and Voltage Collapse .......................................... 14

$2.1 \quad$ Introduction ...................................................................................................... 14

2.2 Developed polar line-wise PF system of equations ....................................... 15

2.3 Polar-form Line-Wise Power Flow Algorithm ................................................. 19

2.4 Development of the Jacobian Matrix............................................................ 23

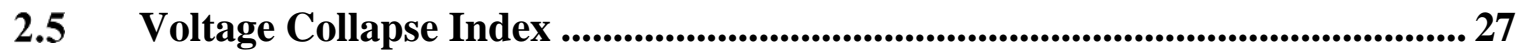


2.6 Results of System Studies.............................................................................................. 29

$2.7 \quad$ Discussion on Power Flow ................................................................................................... 32

2.8 Benefits of the Proposed Method .................................................................................... 34

2.9 Performance of Voltage Collapse Index ...................................................................... 35

2.10 Voltage Stability Contingency Analysis ...................................................................... 39

2.11 Summary .................................................................................................................................... 41

Chapter 3: Rectangular Line-Wise Power Flow ................................................................................ 43

3.1 Proposed line-wise PF system of equations ................................................................ 43

$3.2 \quad$ Proposed algorithm ....................................................................................................... 46

3.3 Development of the Jacobian Matrix......................................................................50

3.4 Results of System Studies..........................................................................................56

3.5 Discussion on Power Flow .................................................................................................. 58

3.6 Benefits ................................................................................................................................ 62

$3.7 \quad$ Summary ......................................................................................................... 62

Chapter 4: Line-Wise Optimal Power Flow Using Successive Linear Optimization

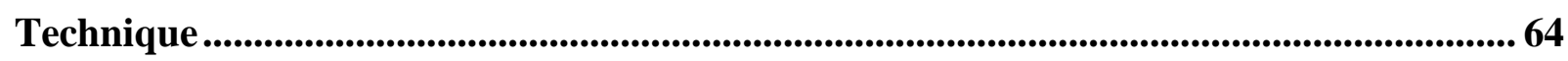

$4.1 \quad$ Introduction ......................................................................................................................... 64

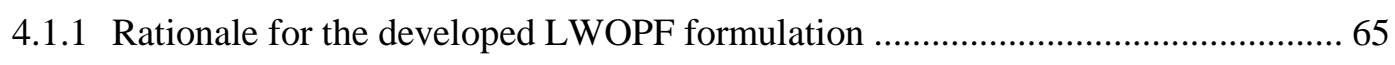

4.2 Line-wise Optimal Power Flow Formulation ......................................................... 66

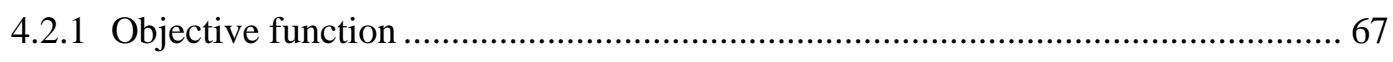

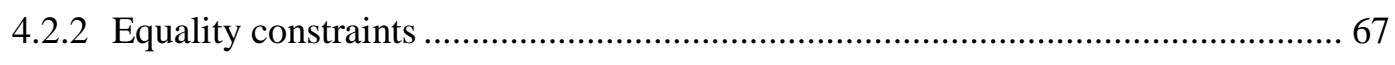

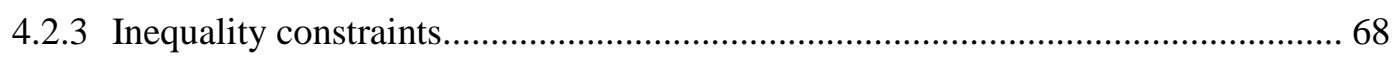

4.2.4 Generic form of nonlinear LWOPF optimization formulation ............................. 69

4.3 Incremental Formulation for SLP Method ............................................................... 70

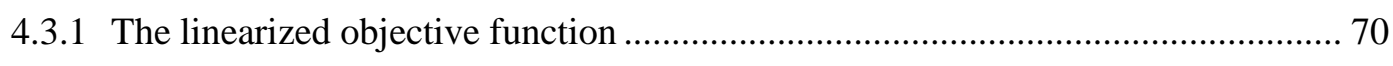




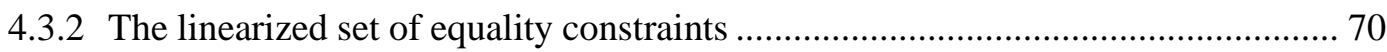

4.3.3 The linearized set of inequality constraints ...................................................... 71

4.3.4 Generic form of linearized LWOPF optimization problem............................... 72

4.4 Successive Linear Programming Algorithm ................................................. 72

4.5 Results of System Studies......................................................................... 74

4.5.1 Performance of the LWOPF method-Objective Function Value and Execution Time 74

4.5.2 Convergence characteristics of proposed algorithm......................................... 75

4.5.3 Comparison between the proposed algorithm with an existing SLP algorithm of

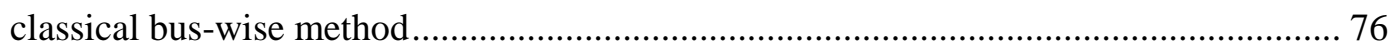

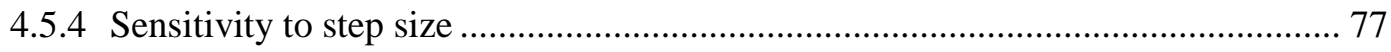

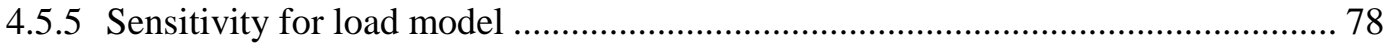

4.5.6 Sensitivity for considering the LWPF solution in each iteration of SLP .............. 80

4.5.7 Sensitivity for initialization methods............................................................. 82

4.6 Discussion on Optimal Power Flow ...................................................................... 83

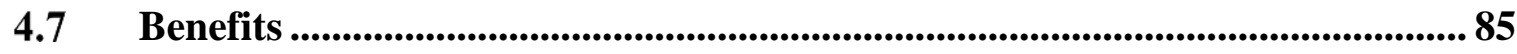

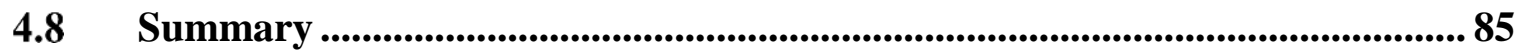

Chapter 5: Voltage Stability Constrained Line-wise Optimal Power Flow ........................ 87

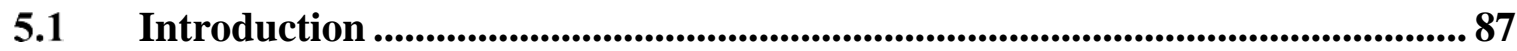

$5.2 \quad$ Line-wise Optimal Power Flow Formulation ......................................................... 88

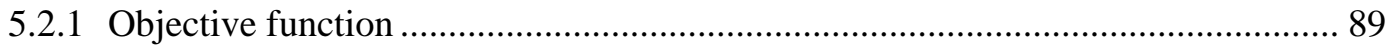

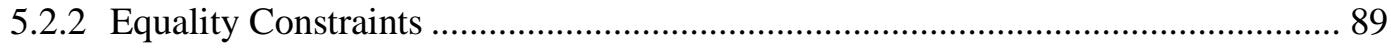

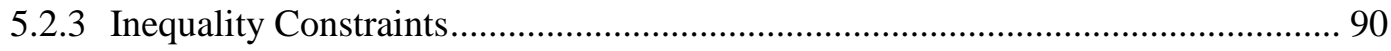

5.3 The Maximum loadability Factor (MLF) of branches....................................... 92

5.4 Results of System Studies ( VSCLWOPF) ................................................. 94

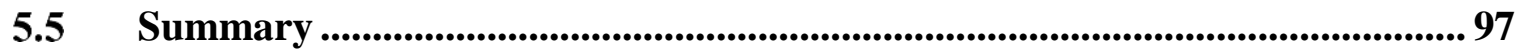


Chapter 6: Successive Linear Optimization - Voltage Stability Incorporation with Linewise Optimal Power Flow ...............................................................................................99

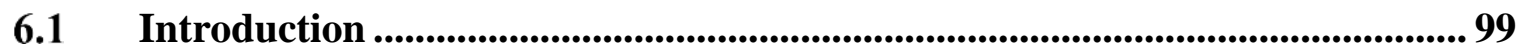

6.2 Nonlinear Line-wise Optimal Power Flow Formulation and the Line Voltage Collapse Index.......................................................................................................... 100

6.2.1 Line-wise power flow formulation (LWPF) and Related Constraints.................. 100

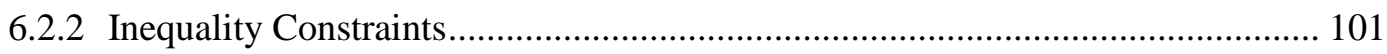

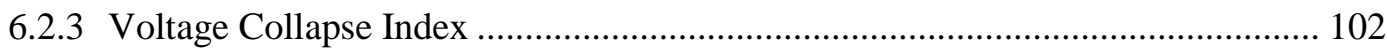

6.2.4 Full Non-linear Formulation of Voltage stability incorporation with LWOPF-model $\# 1$

6.2.5 Full Non-linear Formulation of Voltage stability incorporation with LWOPF-model \#2

6.3 Development of Linear Models of Objective Function, Equality Constraints, Inequality Constraints and VCI indices - models 1 and 2..................................... 104

6.3.1 Linearized Objective Function - model \#1 ................................................ 104

6.3.2 Linearized Objective Function - model \#2 …................................................... 104

6.3.3 Linearized Set of Equality Constraints …...................................................... 105

6.3.4 Linearized Set of Inequality Constraints .......................................................... 105

6.3.5 Linearized Voltage Collapse Indices for Model \#1 only ................................... 106

6.4 Two proposed Linear Models of Voltage Stability incorporation with LWOPF 107

6.4.1 Linear Voltage Stability incorporation with LWOPF- Model \#1 (VCI as set of constraints)

6.4.2 Linear Voltage Stability incorporation with LWOPF- Model \#2 (VCI as an objective function). 108

6.5 Successive Linear Programming Algorithm........................................................ 108

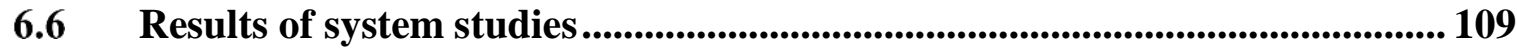




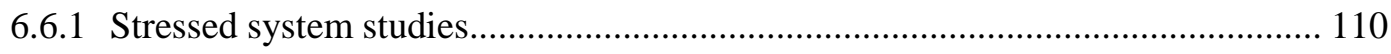

6.6.2 Outage system studies ............................................................................. 114

6.6.3 Comparison with an existing well-known voltage stability constrained OPF (IEEE

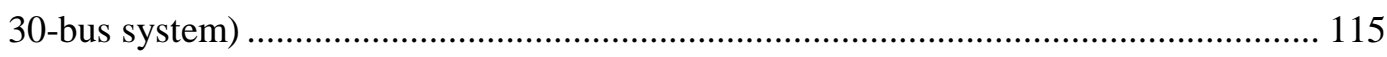

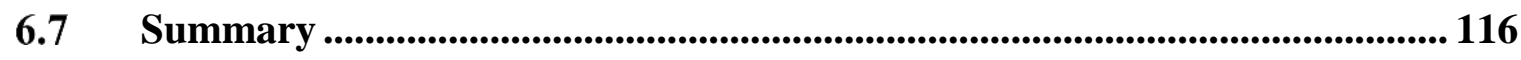

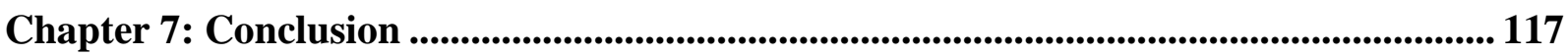

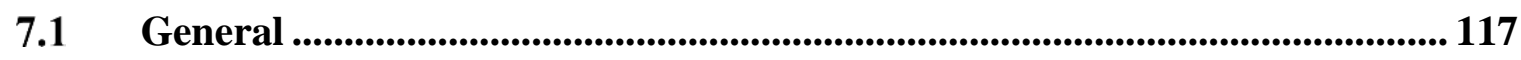

Contribution and main benefits of thesis ...................................................... 118

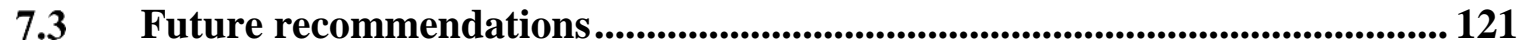

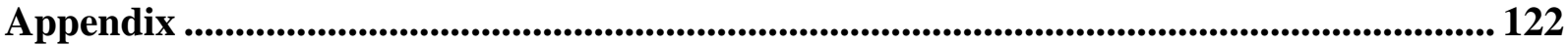

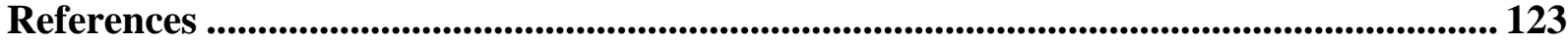




\section{LIST OF TABLES}

Table 2.1 Number of Iterations and Execution Times at $10^{-5}$ per unit tolerance 29

Table 2.2 Comparison of Non-zero Terms of the Line-wise NR and Bus-wise NR method formulations. 32

Table 2.3 Comparison of Jacobian Computations of Line-wise and Bus-wise PF methods using Newton Raphson technique for Non-zero Terms. 33

Table 2.4 Comparison of Size and Condition Number of Jacobian Matrix for Both Line-wise NR and Bus-wise NR methods 34

Table 2.5 Value of Worst VCI and Computation Times for All the VCIs. 38

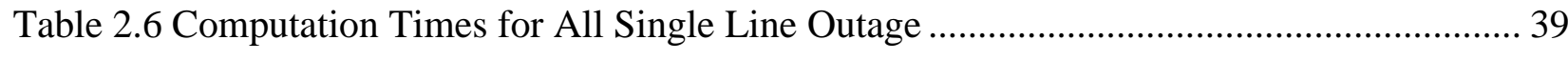

Table 2.7 Twenty Most Severe Single Outage Contingency Cases for Polish 2383-bus System.. 40

Table 3.1 Number of Iterations and Execution Times at $10^{-5}$ per unit tolerance ............................ 58 Table 3.2 Comparison of formulations of Rectangular Forms of Line-wise NR method and Buswise NR method for Non-zero Terms 59

Table 3.3 Comparison of Jacobian Computation of Rectangular Forms of Line-wise and Bus-wise PF using Newton Raphson for Non-zero Terms ...... 60

Table 3.4 Comparison of Size and Condition Number of Jacobian Matrix for Both Rectangular Forms of Line-wise NR method and Bus-wise NR method. 61

Table 4.1 Comparison of Proposed LWOPF Method using three solvers with MATPOWER Objective Function Value and Execution Time 75

Table 4.2 Comparison between SLP based LWOPF and SLP Based BWOPF .77 Table 4.3 Performance Comparison between SLP based LWOPF Solutions with Various Load Models 80

Table 6.1 Comparison between the results of two proposed models and normal LWOPF for a line outage of Ward-Hale 6-bus system. 114 Table 6.2 Comparison between the results of two proposed models and normal LWOPF with best results of an existing VSCOPF for a stressed IEEE 30-bus system at 1.4 load factor. 115 Table 6.3 Comparison between the results of two proposed models and normal LWOPF with best results of an existing VSCOPF for a IEEE 30-bus system with line outage for (L2-5). 116 


\section{LIST OF FigURES}

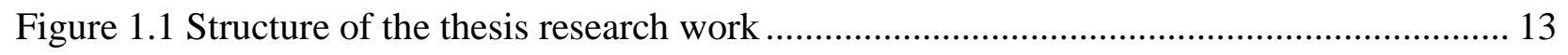

Figure 2.1 Series impedance element of the pi-model of the lth transmission branch connecting

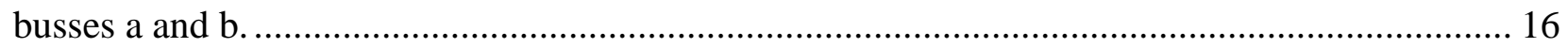

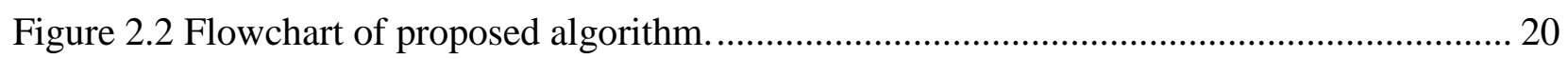

Figure 2.3 Plot of 6-bus System Jacobian with 40 rows and 40 columns................................. 27 Figure 2.4 The Root Mean Square Error (RMSE) of bus voltage magnitudes and angles for difference in results between PF solutions of the traditional bus-wise NR method and the proposed

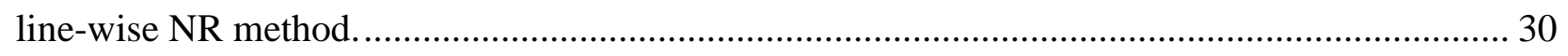

Figure 2.5 Convergence characteristic of the proposed method for different test systems........... 31

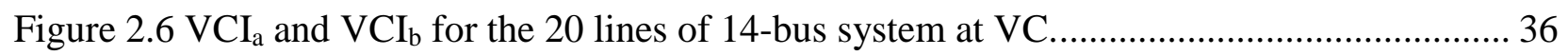

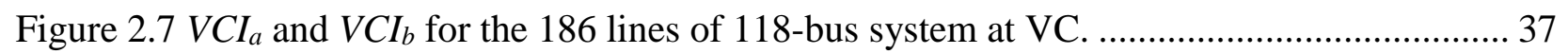

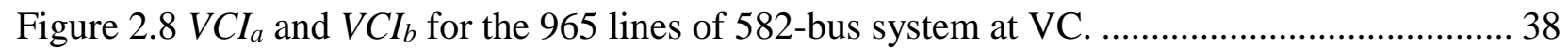

Figure 3.1 Series impedance element of the pi-model of the $l^{\text {th }}$ transmission branch connecting

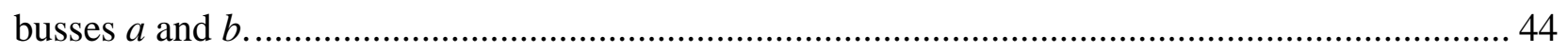

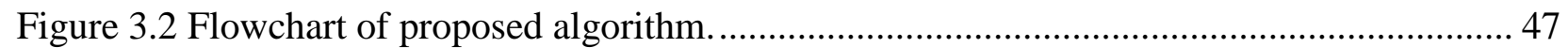

Figure 3.3 Plot of 6-bus System Jacobian matrix............................................................. 55

Figure 3.4 Spy matrix of 118-bus System Jacobian matrix. ................................................. 55

Figure 3.5 The Root Mean Square Error (RMSE) of bus real and imaginary voltage terms for difference in results between PF solutions of the traditional rectangular bus-wise and the proposed

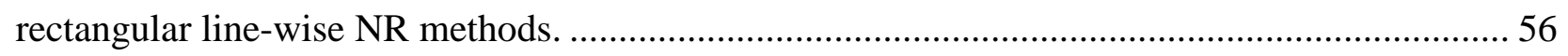

Figure 3.6 Convergence characteristic of the proposed method for different test systems........... 57 Figure 4.1 Series impedance element of the pi-model of the $l^{\text {th }}$ transmission branch connecting buses

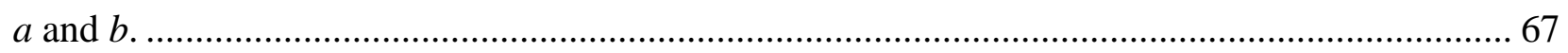

Figure 4.2 Flowchart of proposed LWOPF based on SLP................................................ 73

Figure 4.3 Convergence characteristics of the proposed method for different test systems. ........ 76 Figure 4.4 Convergence characteristics of proposed LWOPF-SLP solution for IEEE 118-bus power

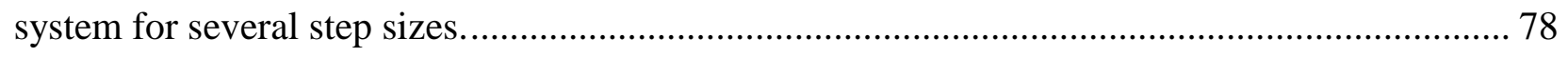


Figure 4.5 Ratio between execution times of LWPF start to flat start in percentage at different step

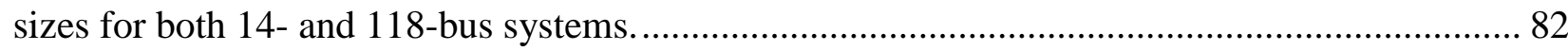
Figure 5.1 Series impedance element of the pi-model of the lth transmission branch connecting

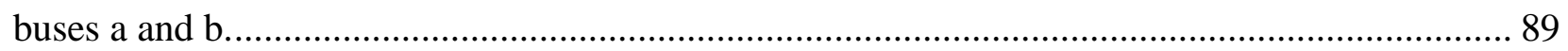

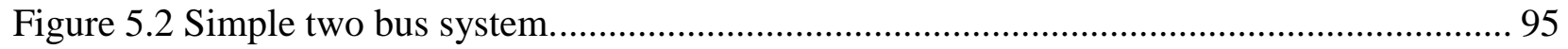

Figure 5.3 Total generation cost, PGC, and PGE values with increasing of MLF. ................... 96

Figure 5.4 Percentage change of the objective cost function after and before considering of MLF

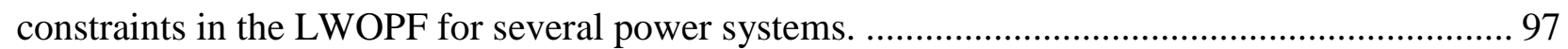

Figure 6.1 Series impedance element of the pi-model of the lth transmission branch connecting

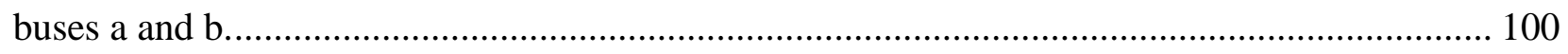

Figure 6.2 Flowchart of proposed LWOPF based on SLP................................................ 109 Figure 6.3 Comparison between cost function value of the two proposed models and the normal LWOPF of Ward-Hale 6-bus system at several loading factors (stressed)............................. 111 Figure 6.4 Comparison between the value of minimum VCI of the solution of the two proposed models and the normal LWOPF of Ward-Hale 6-bus system at several loading factors (stressed).

Figure 6.5Comparison between the cost function value of the solution of the two proposed models and the normal LWOPF of 14-bus system at several loading factors (stressed)...................... 112 Figure 6.6 Comparison between the value of minimum VCI of the solution of the two proposed models and the normal LWOPF of 14-bus system at several loading factors (stressed)........... 112 Figure 6.7 Comparison between the cost function value of the solution of the two proposed models and the normal LWOPF of 118-bus system at several loading factors (stressed).................... 113 Figure 6.8 Comparison between the value of minimum VCI of the solution of the two proposed models and the normal LWOPF of 118-bus system at several loading factors (stressed).......... 113 Figure 6.9 Enhancement of bus voltages of the two proposed models compared to the normal LWOPF of Wald-Hale 6-bus system with a line outage contingency. 114 
NOMENCLATURE

\begin{tabular}{|c|c|}
\hline$a$ & Index of sending end buses of branches (from bus) \\
\hline$b$ & Index of receiving end buses of branches (to bus) \\
\hline$g$ & Index of generation buses \\
\hline$i$ & Index of buses \\
\hline$k$ & Index of successive linear programming iteration \\
\hline$l$ & Index of branches (transmission lines or transformers) \\
\hline A & Constraint matrix of the incremental LWOPF problem \\
\hline $\bar{b}, \underline{b}$ & Upper and lower limits of constraints of the incremental LWOPF problem \\
\hline$B S$ & $\begin{array}{l}\text { Vector of bus-wise susceptance elements including those of pi-models of } \\
\text { lines and transformers }\end{array}$ \\
\hline $\mathrm{C}$ & Cost vector of the incremental LWOPF problem \\
\hline$d$ & Target vector in a generic equality constraint of a generic OPF formulation \\
\hline$e$ & Vector of real voltage term \\
\hline$f$ & Vector of imaginary voltage term \\
\hline$f_{\circ}$ & The objective function of a generic OPF formulation \\
\hline$F 1(w w)$ & Vector of first set of line functions (first end) in rectangular form \\
\hline$F 2(w w)$ & Vector of second set of line functions (second end) in rectangular form \\
\hline$F 3(w w)$ & Vector of third set of line functions (first end) in rectangular form \\
\hline$F 4(w w)$ & Vector of fourth set of line functions (second end) in rectangular form \\
\hline$F 5(w w)$ & Vector of functions for bus real powers in rectangular form \\
\hline$F 6(w w)$ & Vector of functions for bus reactive powers in rectangular form \\
\hline$F 7(w w)$ & vector of functions for square of voltage buses in rectangular form \\
\hline$F 8(w w)$ & Set of non-linear equations that models a power system in rectangular form \\
\hline$F A(w)$ & Vector phase angle relations (first end) in polar form \\
\hline$F B(w)$ & Vector of phase angle relations (second end) in polar form \\
\hline$F F(w)$ & Vector of line-based voltage equations (first end) in polar form \\
\hline
\end{tabular}




\begin{tabular}{|c|c|}
\hline$f_{g}$ & The set of equality constraints of a generic OPF formulation \\
\hline$f_{h}$ & The set of inequality constraints of a generic OPF formulation \\
\hline$F P(w)$ & Vector of bus-wise real powers injections from lines in polar form \\
\hline$F Q(w)$ & Vector of bus-wise reactive power injections from lines in polar form \\
\hline$F S(w)$ & Vector of line-based voltage equations (second end) in polar form \\
\hline$F T(w)$ & Set of non-linear equations that models a power system in polar form \\
\hline$G S$ & $\begin{array}{l}\text { Vector of bus conductance elements including those of pi-models of lines } \\
\text { and transformers }\end{array}$ \\
\hline$g s_{i k}, b s_{i k}$ & The real and imaginary terms of the element $y_{i k}$ of bus admittance matrix \\
\hline$\underline{h}, \bar{h}$ & $\begin{array}{l}\text { Upper and lower bounds on a generic inequality of a generic OPF } \\
\text { formulation }\end{array}$ \\
\hline$J$ & Line-wise jacobian matrix \\
\hline$[M]$ & Modified bus incidence matrix with a dimension of $N B \times 2 \cdot N T$ \\
\hline $\begin{array}{l}M L F F_{l} \\
M L F S_{l}\end{array}$ & Maximum loadability factors at both ends of branch $l$ \\
\hline$N B$ & Total number of buses in the power system \\
\hline$N G$ & Total number of generators buses \\
\hline$N L B$ & Total number of load buses \\
\hline$n p, n q$ & $\begin{array}{l}\text { Exponent constant of exponential active and reactive load model } \\
\text { respectively }\end{array}$ \\
\hline$N T$ & Total number of branches \\
\hline$p_{1}, p_{2}, p_{3}$ & Constants of polynomial (ZIP) active power model \\
\hline$P D$ & Vector of active load demand power \\
\hline$P F$ & Vector of first end active powers flowing in branches, \\
\hline$P G$ & Vector of active generated power, \\
\hline$\overline{P G_{g}}, \underline{P G_{g}}$ & Upper and lower limit for active power generation of generator $g$ \\
\hline$\overline{\Delta P G_{g}}, \underline{\Delta P G_{g}}$ & $\begin{array}{l}\text { Incremental upper and lower step bounds for active power generation of } \\
\text { generator } g\end{array}$ \\
\hline
\end{tabular}




\begin{tabular}{|c|c|}
\hline$P S$ & Vector of second end active powers flowing in branches \\
\hline$q_{1}, q_{2}, q_{3}$ & Constants of polynomial (ZIP) reactive power model \\
\hline$Q G$ & Vector of reactive generated power \\
\hline$\overline{Q G_{g}}, \underline{Q G_{g}}$ & Upper and lower limit for reactive power generation of generator $\mathrm{g}$ \\
\hline$\overline{\Delta Q G_{g}}, \underline{\Delta Q G_{g}}$ & $\begin{array}{l}\text { Incremental upper and lower step bounds for reactive power generation of } \\
\text { generator } g\end{array}$ \\
\hline$Q D$ & Vector of reactive load demand power \\
\hline$Q F$ & Vector of first end reactive powers flowing in branches \\
\hline$Q S$ & Vector of second end reactive powers flowing in branches \\
\hline$R$ & Vector of resistance of lines and transformers \\
\hline$S F \angle \emptyset F$ & Vector of first end of complex power flowing in branches \\
\hline$S_{l}$ & Apparent power flow of transmission line $l$ \\
\hline$\overline{\Delta S L_{l}}$ & Incremental upper step bound for apparent power flow of transmission line $l$ \\
\hline$\overline{S L_{l}}$ & Upper limit for apparent power flow of transmission line $l$ \\
\hline$S R$ & Target vector in NR power flow formulation in the rectangular form \\
\hline$S S \angle \varnothing S$ & Vector of second end of complex power flowing in branches \\
\hline ST & Target vector in NR power flow formulation in the polar form \\
\hline$U$ & Vector of the square of bus voltage magnitudes \\
\hline$\overline{U_{i}}, \underline{U_{i}}$ & Upper and lower limit for the square of voltage magnitudes of bus $i$ \\
\hline$\overline{\Delta U_{i}}, \underline{\Delta U_{i}}$ & $\begin{array}{l}\text { Incremental upper and lower step bounds for the square of bus voltage } \\
\text { magnitudes of bus } i\end{array}$ \\
\hline$V$ & Vector of bus voltage magnitudes \\
\hline $\mathrm{VCI}_{\mathrm{a}}, \mathrm{VCI}_{\mathrm{b}}$ & Voltage collapse indices at both sides \\
\hline$\overline{V_{i}}, \underline{V_{i}}$ & Upper and lower limit for the voltage magnitudes of bus $i$ \\
\hline$V_{S H}$ & Vector of generation bus voltage magnitude \\
\hline $\boldsymbol{W}$ & 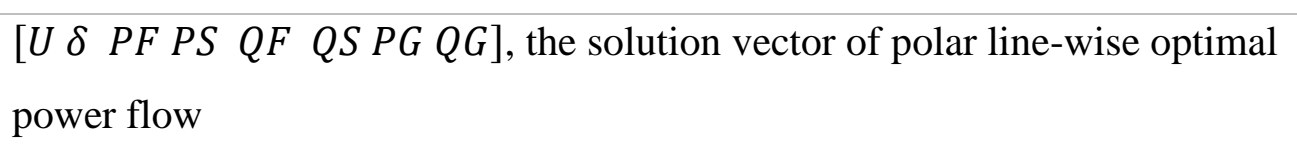 \\
\hline$w$ & {$\left[\begin{array}{llllll}U & \delta & P F & P S & Q F & Q S\end{array}\right]$, the solution vector of polar line-wise power flow } \\
\hline
\end{tabular}




\begin{tabular}{|c|c|}
\hline$\Delta \boldsymbol{W}$ & $\begin{array}{l}{\left[\Delta U, \Delta \delta, \Delta P_{a}, \Delta P_{b}, \Delta Q_{a}, \Delta Q_{b}, \Delta P_{G}, \Delta Q_{G}\right]^{T}, \text { solution vector of incremental }} \\
\text { line-wise optimal power flow optimization problem }\end{array}$ \\
\hline$W_{c}$ & {$[U P G]$, the control vector of polar line-wise optimal power flow } \\
\hline$W_{d}$ & $\begin{array}{l}{\left[\begin{array}{lllll}\delta & P F & P S & Q F & Q S\end{array}\right] \text {, the dependent vector of polar line-wise optimal }} \\
\text { power flow }\end{array}$ \\
\hline$w w$ & $\begin{array}{l}\text { [e } f \text { PF PS } Q F \quad Q S \text {, the solution vector of rectangular line-wise power } \\
\text { flow }\end{array}$ \\
\hline$X$ & Vector of inductive reactance of lines and transformers \\
\hline$y_{i k} \angle \vartheta_{i k}$ & An off-diagonal element of bus admittance matrix between buses $i$ and $k$ \\
\hline$Y$ & Admittance matrix of classical bus-wise power flow \\
\hline$Y_{S}$ & Shunt admittance of transmission line pi-model \\
\hline$Z \angle \theta$ & Vector of impedance of lines and transformers \\
\hline$\tau$ & Minimum targeted value of MLF \\
\hline$\gamma$ & Mismatch tolerance of SLP algorithm \\
\hline$\beta$ & Terms of solution roots of quadratic equation of $U$ \\
\hline$\alpha$ & Step size of SLP technique \\
\hline$\delta$ & Vector of bus voltage phase angles \\
\hline
\end{tabular}




\section{Chapter One}

\section{CHAPTER 1: INTRODUCTION}

\subsection{Introduction}

Power flow (PF) is the most frequently performed analysis in power systems engineering. It represents the backbone of almost all the power system analysis studies in the academic, industrial and utility sectors. Any improvement in the PF formulation and solution methods is of immense value for several technical and economic reasons. The reasons for benefits are multifaceted. First, are its the economic ramifications. The non-convex nature of the space modeled by the existing power balance equations results in power system optimization formulations that are also non-convex. These non-convex power systems optimization formulations yield suboptimal solutions, each of which represents one of the several local optimum solutions. Second, there are technical considerations. The solution time for power balance equations depends on the formulation and the complexity of terms in the Jacobian of power balance equations (i.e., order and nonlinearity). Improvements in reducing complexity of the Jacobian terms of power balance equations will yield significant benefits in achieving faster and more accurate solutions for power balance equations. Third, interest in power balance equations arises from their attributes, such as indication of system voltage collapse. Their Jacobian can readily indicate the state of power systems and proximity to voltage collapse. However, attributes elicited from the Jacobian of power balance equations is very much dependent upon the formulation of power balance equations. Hence, development of simpler and faster power balance equations that directly indicate proximity to voltage collapse is very beneficial.

In this context, the first topic of interest in this research work is to develop an efficient simple PF algorithm considering the lines instead of buses. The set of line-wise equations and the Jacobian matrix are developed and followed by a solution method using Newton Raphson (NR) technique. The developed formulations are in both rectangular and polar forms. As a by-product, voltage collapse (VC) analysis can be conducted by developing a suitable voltage collapse index. The proposed method can be used to reveal the critical lines most susceptible to VC and show that they are directly available in the Jacobian of the linewise NR method, without additional calculations.

The second topic of interest in this work is the development of an OPF formulation based on the linewise power balance equations. The formulation may be explored for solution using classical linear and non- 
linear optimization techniques. These formulations, given the model of solution space, will be tested for improved solutions and possible advancement to global solutions.

The third topic of interest is to ensure voltage stability. Several models for incorporating voltage stability are explored in conjunction with line-wise optimal power flow.

\subsection{Survey of Recent work}

In this section, a brief literature survey is presented as follows.

\subsubsection{Bus-wise power flow}

Power flow (PF) analysis refers to solution of a set of equations that describe a power system, yielding bus-wise voltage solutions for the whole power system. PF analysis plays a vital role in many aspects of electrical power systems engineering. PF analysis constitutes the basis for studies in topics such as power systems optimization, power systems planning, voltage stability, etc. The most popular formulation for PF analysis considers a set of bus-wise power balance equations based on nodal analysis. The formulation is solved using numerical techniques, such as Gauss-Seidel, Newton-Raphson (NR), and their variants, such as fast-decoupled power flow. The solution yields bus-wise voltage values for the power system. Line flows are computed using impedance of the network elements and computed voltage values.

Over the past several decades, an enormous effort has been devoted to the development of efficient and accurate solution methods for solving the set of bus-wise power balance equations. In 1935, an elementary solution for PF analysis was presented in [1]; then, it was followed by several attempts to solve the problem [2-7]. The first powerful procedure for solving the bus-wise PF problem based on the NR method was introduced in 1967 [8], and its variant, the fast decoupled method was presented later in 1974 [9].

This was followed by several studies concerning the singularity of the Jacobian matrix; the existence, uniqueness, convergence, and computational efficiency of PF solutions were also studied in [10-15]. In [10], the relation between multiple PF solutions and voltage instability was considered. A method to solve the PF for large weakly meshed power system is presented in [11]. Galiana et al. studied the effect of the Jacobian singularity on the solution of PF in [12]. It was followed by several attempts to improve the PF solution performance as in [13] and [14]. In [15], an adaptive preconditioner algorithm was developed and applied on PF calculation. The literature contains other PF studies considering three-phase unbalanced conditions 
[16-18]. In [16], the sequence component frame was used to solve three-phase power flow. Zhang et al. developed a solution algorithm for unbalanced three-phase PF using symmetrical components. In [18], three-phase PF solution algorithm was presented and used for real-time distribution system.

Recently, interest in meshed and radial distribution networks, especially with renewable energy resources and microgrids, have rekindled [19-23]. In [19], an algorithm of PF solution was developed for islanded microgrid based on newton trust region method. PF solution algorithm for distribution networks with high penetration of rooftop solar PV was presented in [20]. It was followed by numerous attempts to solve the PF problem on distribution level as in [21] based on Fortescue transformation. A method based on impedance bus was developed to solve the PF for large unbalanced distribution systems in [22]. Garces [23] presented a linear formulation and solution algorithm for three-phase PF of distribution systems.

The literature shows that both polar and rectangular formulations of bus-wise power flow are considered. In [8] and [24], development and comparison between the two polar and rectangular PF formulations are performed based on NR solution method. Many research works have been pursued within the context of rectangular PF coordinates [25-28]. Stott presented a comprehensive review on the existing PF method up to 1975 including the rectangular PF formulations in [25]. In [26], a rectangular nonlinear

formulation of PF was developed. A rectangular PF formulation is expressed using Taylor series including derivatives up to the second order in [27]. In 2002, interest in rectangular form of power flow was rekindled when Exposito et al. reformulated the existing of rectangular PF formulation in terms of bus voltage and currents in [28].

\subsubsection{Polar bus-wise power balance equations}

Hereunder, the conventional polar form of bus-wise power balance equations will be presented. Consider a power system with $N G$ generator buses and $N L B$ load buses, such that the total number of buses $N B=$ $N G+N L B$, and $N T$ is the total number of transmission branches. The bus admittance matrix is $Y_{N B \times N B}$. The total active and reactive power ( $P T$ and $Q T)$ at bus k can be expressed as follows:

$$
\begin{array}{ll}
P T_{k}(V, \delta)=P G_{k}-P D_{k}=V_{k} \cdot \sum_{i=1}^{N B} V_{i} \cdot y_{i k} \cdot \cos \left(\delta_{k}-\delta_{i}-\vartheta_{k i}\right) & \forall k \in N B \\
Q T_{k}(V, \delta)=Q G_{k}-Q D_{k}=V_{k} \cdot \sum_{i=1}^{N B} V_{i} \cdot y_{i k} \cdot \sin \left(\delta_{k}-\delta_{i}-\vartheta_{k i}\right) & \forall k \in N B
\end{array}
$$


It must be noted that the following are known:

(1) voltage magnitude and phase angle at the slack bus;

(2) real power injection (generation minus load) and bus voltage magnitudes at all generation busses; and

(3) real and reactive power injections (negative of real and reactive power loads) at load busses.

The unknowns are then:

(1) real and reactive power injections at the slack bus;

(2) bus voltage phase angles and reactive power injections at generator busses; and

(3) voltage magnitudes and phase angles at load busses.

Summarized in a tabular form below:

\begin{tabular}{c|c|c}
\hline & Knowns & Unknowns \\
\hline \multirow{2}{*}{ Slack Bus } & $V$ & $P T=P G-P D$ \\
& $\delta$ & $Q T=Q G-Q D$ \\
\hline \multirow{2}{*}{ Generator Bus } & $P T=P G-P D$ & $Q T=Q G-Q D$ \\
& $V$ & $\delta$ \\
\hline \multirow{2}{*}{ Load Bus } & $P T=-P D$ & $V$ \\
& $Q T=-Q D$ & $\delta$ \\
\hline
\end{tabular}

\subsubsection{Rectangular bus-wise power balance equations}

In this subsection, the conventional rectangular form of bus-wise power balance equations will be presented. Consider a power system with NG generator buses and NLB load buses, such that the total number of buses $N B=N G+N L B$, and NT is the total number of transmission branches. The bus admittance matrix is $Y_{N B \times N B}$. The off-diagonal element of admittance matrix $y_{i k} \angle \vartheta_{i k}=g s_{i k}+j \cdot b s_{i k}$

$$
\begin{array}{r}
P T_{k}(e, f)=P G_{k}-P D_{k}=\sum_{i=1}^{N B}\left[e_{k} \cdot\left(e_{i} \cdot g_{i k}-f_{i} \cdot b s_{i k}\right)+f_{k} \cdot\left(e_{i} \cdot b_{i k}+f_{i} \cdot g s_{i k}\right)\right] \\
\forall k \in N B \\
Q T_{k}(e, f)=Q G_{k}-Q D_{k}=\sum_{i=1}^{N B}\left[f_{k} \cdot\left(e_{i} \cdot g_{i k}-f_{i} \cdot b s_{i k}\right)-e_{k} \cdot\left(e_{i} \cdot b_{i k}+f_{i} \cdot g s_{i k}\right)\right] \\
\forall k \in N B
\end{array}
$$


It must be noted that the following are known:

(1) real and imaginary terms of voltage at the slack bus;

(2) real power injections (generation minus load) and bus voltage magnitudes at all generation busses; and,

(3) real and reactive power injections (negative of real and reactive power loads) at load busses.

The unknowns then are:

(1) real and reactive power injections at the slack bus;

(2) real and imaginary terms of voltage as well as the reactive power injections at generator busses; and,

(3) real and imaginary terms of voltage at load busses.

Knowns and unknowns are summarized in a tabular form below:

\begin{tabular}{c|c|c}
\hline & Knowns & Unknowns \\
\hline \multirow{2}{*}{ Slack Bus } & $e$ & $P T=P G-P D$ \\
& $f$ & $Q T=Q G-Q D$ \\
\hline \multirow{2}{*}{ Generator Bus } & $P T=P G-P D$ & $Q T=Q G-Q D$ \\
& $V_{S H}$ & $e$ and $f$ \\
\hline \multirow{2}{*}{ Load Bus } & $P T=-P D$ & $e$ \\
& $Q T=-Q D$ & $f$ \\
\hline
\end{tabular}

\subsubsection{Voltage Collapse}

Voltage collapse (VC) is a localized dynamic phenomenon occurring across power lines due to a lack of reactive power; it is the most severe stage of voltage instability, leading to a low-voltage profile in a significant part of the power system. The event can propagate and eventually lead to a blackout [29, 30]. As reported in [31], steady state voltage stability analysis is more comprehensive and can provide much insight to the voltage collapse problem. In this context, several approaches have been employed in the literature for static analysis of voltage stability, such as P-V and Q-V curves, modal analysis, continuation power flow, minimum singular value based on the Jacobian matrix, bifurcation theory, and energy function, to name a few. 
The P-V and Q-V curves are the most widely used methods of voltage stability analysis. To generate these curves, a sizeable number of power flow (PF) computations have to be executed. The curves are used to identify the "knee" point that represents the stability limit. However, such methods require a huge computational burden and, consequently, take a long time [29, 30]. Modal analysis is used in [31] for steady state voltage stability analysis based on the eigenvalues of the Jacobian matrix. Positive eigenvalues of the Jacobian matrix indicate a stable system, while a negative eigenvalue shows that the system is unstable. However, as the demand load is increased toward the maximum loading condition, the Jacobian matrix suffers from the singularity problem, which will prevent the PF solution from convergence [29-31].

As a solution for the singularity of Jacobian matrix, the continuation PF is presented in [32-34]. The solution of continuation power flow was based on homotopy techniques. The method is solved in a two-step process, with a predictor step and a corrector step. It considers an additional variable called the load-increase continuation parameter, which increases the size of the Jacobian matrix by one.

As a result, the Jacobian matrix becomes non-singular with a suitable choice of the continuation parameter. However, the continuation PF still deals with the singular Jacobian matrix during the corrector step [35]. In [36], the minimum singular value of the Jacobian matrix was used for a proximity index for the voltage instability. The energy function concept was used to provide a localized measure of voltage security in [37]. Also, the the limitations and applications of saddle-node bifurcation theory in voltage collapse analysis were presented in [38].

Some other approaches utilize local measurements to develop a voltage collapse indicator [39-41]. In [42], a PF method to eliminate the Jacobian matrix singularity and compute the voltage stability margins is presented. However, bus-wise PF methods and the associated Jacobian matrix cannot readily point out the line, or a set of lines, that is the most susceptible to voltage collapse. This inability persists as a distinct shortcoming of voltage collapse assessment methods that rely on bus-wise PF methods.

\subsubsection{Optimal power flow}

Optimal power flow (OPF) is an optimization problem that aims at finding the optimal solution for an objective, such as minimizing the total generating cost, while satisfying constraints such as power balance equations, bus voltage limits, line flow limits, etc. The objective of OPF may vary depending on the nature of the study. Solving the OPF for a power system operator, every 5 minutes or in a similar time frame, remains a fundamental task. It has significant technical and economic importance and benefits. Technical important is due to its key role in maintaining a power system's operation within feasible and safe zones, 
observing all constraints. Economic importance is due to its key role in ensuring that the power system is operated in the most economic manner.

The main applications of the OPF tool include: (1) scheduling the power system at a frequent interval, as often as every five minutes [43]; (2) dual variables of OPF formulation provide price signals for resources and equipment limits; (3) OPF and its variants are used as a basic tool for assessing various investment decisions; and; (4) OPF and its variants form a subset of formulations for unit commitment, optimal planning, and such other applications.

The work devoted in the area of OPF could be mainly classified based on (1) the formulation type, which could be nonlinear or linear, considering power balance equations in its nonlinear form (AC formulation) or the linear form (DC formulations) respectively, and, (2) the solution algorithm which could be successive linear optimization or nonlinear optimization such as quadratic optimization, conic optimization or metaheuristic optimization techniques to name a few.

The literature shows that many research efforts have been devoted to the development of efficient and accurate solution approaches for solving the OPF problem [44, 45]. The first formulation of the OPF problem was introduced in 1962 [46]. It was followed by several attempts to solve the problem [47-50]. A powerful solution algorithm for OPF based on a reduced gradient method is presented in [51]. In [52-55], the OPF was solved using quadratic programming approaches. Metaheuristic algorithms have also been applied to solve the OPF [56-60].

Recently, several solution algorithms tried to convexify the classical formulation and solve the resultant relaxation using semidefinite programming (SDP) [61-68]. In addition, interests in distribution networks with OPF, especially with renewable energy resources, energy storage, and microgrids, have been presented in [69-72].

The first application of SDP in power system analysis was presented in [73] as relaxations for economic dispatch and unit commitment problems. In [74], the hydrothermal coordination problem was solved using SDP. Jabr formulated the DC-OPF and the OPF of a radial distribution system as conic optimization problems in [75, 76] respectively. The OPF problem was presented and solved using SDP, with shortcomings on large systems [77]. In 2011, the graph partitioning technique was employed with SDP to solve the OPF problem with better computing performance, but it still cannot be applied for large systems [78]. In [63], the duality of the classical OPF problem was developed in an SDP model which considers the zero-duality-gap condition of global optimality. In a two-part paper [79, 80], the branch flow technique was used for convexification of the OPF problem for both radial and mesh networks. In addition, the global 
optimality condition of OPF relaxation was derived from the Karush-Kuhn-Tucker (KKT) conditions in [62]. With a focus on structural properties rather than algorithms of SDP relaxation of OPF, the two-part paper in $[64,81]$ provided conditions for the exactness of OPF convex relaxations.

Many other works have been devoted to improving the efficiency and performance of SDP relaxation of the OPF problem [65-68, 82-91]. Some other approaches employed SDP relaxation for optimal scheduling of energy storage units and renewable resources $[92,93]$. Nevertheless, existing convex optimization solution algorithms still have limitations, which emphasizes the need to develop efficient global methods in solving OPF problems [94].

Although the first OPF problem was formulated 55 years ago [46], there is still no robust solution, due to the nonlinear and nonconvex nature of its formulation. Practical approaches to solving nonconvex problems do not yet exist, and even the SDP relaxations of OPF still have some limitations [94]. As a result of that, OPF is still a major research emphasis given its technical and economic importance in operation of modern power systems.

The reliability, robustness and relative speed of linear programming (LP) techniques make its approaches among the most extensively used methods to solve the OPF formulation in the literature [95]. Successive linear programming (SLP) technique was employed for solving the power system scheduling problems in general and as one of the most popular approaches for OPF [96-108]. SLP was used for real and reactive power optimization in [95] - [97].

Literature shows that some SLP approaches have used the accurate AC power flow equations in a separate stage as in [102-105], while others have used the approximate DC power flow model such as [99101]. Some approaches employed the interior point methods (IPM) to solve the LP problem [104, 105], which demonstrated faster convergence than the Simplex algorithm.

Recently, the interest in using SLP for solving OPF has been rekindled [106-108]. In [106], an OPF model with environmental constraints was developed and solved using SLP. Yang et al. presented an approximated OPF model which is based on the quasi-linear relationship of P- $\theta$ and solved by SLP [107]. The quadratic apparent branch flow limits were linearized and a method to recover the AC feasibility was provided. The method is complicated and includes a lot of approximation.

In [108], Castillo et al. developed an OPF model based on the 'IV-ACOPF' formulation. This formulation is based on a current injections approach that linearly couples the quadratic constraints at each bus. The method is associated with a great deal of approximation and penalty factors which may affect the 
solution performance. For the first few iterations, the high-quality initial points could be unavailable, which could make the approximation error relatively large.

\subsubsection{Classical formulations}

In this section, the bus-wise classical OPF formulation will be shown as follows.

Objective Function

$$
\text { Min } \sum_{k \in N_{G}} F\left(P G_{k}\right)=c_{k 2} \cdot P G_{k}^{2}+c_{k 1} \cdot P G_{k}+c_{k 0}
$$

Subject to:

$>$ Generator active power limit

$$
\underline{P G_{k}} \leq P G_{k} \leq \overline{P G_{k}} \quad \forall k \in N G
$$

$>$ Generator reactive power limit

$$
\underline{Q G_{k}} \leq Q G_{k} \leq \overline{Q G_{k}} \quad \forall k \in N G
$$

$>$ Bus voltage limit

$$
\underline{V_{k}} \leq V_{k} \leq \overline{V_{k}} \quad \forall k \in N B
$$

Line power flow limit

$$
\left|S_{l}\right| \leq S_{l}^{\max } \quad \forall l \in N T
$$

Active power balance

$$
P G_{k}-P D_{k}=V_{k} \cdot \sum_{i=1}^{N B} V_{i} \cdot y_{i k} \cdot \cos \left(\delta_{k}-\delta_{i}-\vartheta_{k i}\right) \quad \forall k \in N B
$$


Reactive power balance

$$
Q G_{k}-Q D_{k}=V_{k} \cdot \sum_{i=1}^{N B} V_{i} \cdot y_{i k} \cdot \sin \left(\delta_{k}-\delta_{i}-\vartheta_{k i}\right) \quad \forall k \in N B
$$

\subsubsection{Voltage stability constrained optimal power flow}

Power systems may operate under heavy loading conditions during emergencies, near to the stability margin, and often with a high penetration of renewable resources, and their intermittent nature [109-113]. The incorporation of voltage security into power system operation analysis therefore becomes an urgent demand. The literature shows that voltage security is considered in OPF formulation in two ways. First, the voltage collapse proximity indicator (VCPI) was employed as a constraint in the OPF problem [114-118]. The second approach was to incorporate VCPI as the objective function, where the objective was maximizing the minimum singular value of the Jacobian matrix, as in $[119,120]$. In $[121,122]$, the maximization of the smallest eigenvalue of the non-singular Jacobian matrix has been used as the objective in reactive power dispatch. In addition, the L-index indicator has been used as the objective function in [123-125]. In [126], the improved bus voltage stability index was minimized as an objective function for the purpose of reactive compensation devices planning.

A voltage collapse proximity factor (VCPF) was developed and employed in OPF to ensure the voltage stability. It was implemented in two ways; first, VCPF was incorporated with OPF as a voltage stability constraint and second, it was employed as an objective function [127].

Nevertheless, OPF enforces voltage magnitude and line flow limits. It is still inadequate to ensure the steady-state voltage stability without considering more specific voltage stability constraints [128]. In this context, several voltage stability constrained OPF (VSOPF) formulations have been developed. These VSOPF formulations include load margins and voltage collapse preventing constraints to ensure a secured operation for power systems [127, 129-131]. In [129], an algorithm to identify the maximum distance to voltage collapse was developed and incorporated with OPF.

The previous work was extended to consider a multi-objective function in [130]. A VSOPF was developed as a market-clearing model and solved based on continuation power flow [131]. Chávez and et al. introduced a linear VSOPF model based on DC OPF formulation [132]. Recently, a condition for power 
flow jacobian non-singularity was developed and incorporated with the second-order conic OPF relaxation [133].

\subsection{Motivations of the thesis}

\section{Motivation \#1: $\quad$ Develop a superior power flow method}

Improving the PF analysis, considering problem formulation and solution technique, is an important research exercise. The reasons for that are twofold. First, the nonlinearity and nonconvexity of the existing power balance equations are among the main challenges in solution speed and accuracy. Second, there is a need to improve the PF formulation such that solution space is better.

In this direction, two different options for PF are developed: (1) a polar line-wise power flow formulation; and (2) a rectangular line-wise OPF formulation.

\section{Motivation \#2: $\quad$ Develop a superior optimal power flow method}

In this work, the primary motivation is to develop a novel formulation for OPF that is scalable, efficient and yields optimal solutions closer to global optimum. In this direction, a novel line-wise power flow formulation is developed.

\section{Motivation \#3: Online voltage stability assessment tool}

Guaranteeing adequate voltage security in power systems is an essential objective for power system operators. As mentioned in the introduction and literature survey sections, the voltage collapse and its consequences could have severe impact on power systems. This affirms the need for an online voltage stability assessment tool which can predict the contingencies and help the power system operators to prevent voltage collapse efficiently.

In this work, the main motivation is to maintain voltage stability. The following elements need development: (1) a voltage collapse index which can reveal the critical lines susceptible to voltage collapse, and (2) suitable formulations and algorithms of voltage stability constrained OPF.

\subsection{Objectives of the thesis}

The main objectives of this thesis are as follows.

a) Explore and develop a polar formulation for line-wise power balance equations and a solution method. It is addressed in Chapter 2. 
b) Explore the use of the line-wise PF method for voltage collapse analysis. It is addressed in Chapter 2.

c) Explore and develop a rectangular formulation for line-wise power balance equations and a solution method. It is addressed in Chapter 3.

d) Develop optimal power flow formulations, using the line-wise power flow method, to be solved using classical optimization techniques, such as linear optimization and nonlinear optimization. It is addressed in Chapter 4.

e) Explore and develop the incorporation of voltage stability in the solution of the developed polar formulation of line-wise optimal power flow. It is addressed in Chapters 5 and 6.

\subsection{Summary}

The structure of the developed work is as shown in Figure 1.1. The thesis is organized as follows. In chapter 2, the mathematical formulation of the line-wise PF based on NR method in its polar form and the line voltage collapse index are developed. Further, results comparison with existing well-known methods is presented to show the performance of developed polar line-wise PF. Chapter 3 presents the line-wise PF based on NR in its rectangular form and compares with existing methods. In Chapter 4, a linearized incremental line-wise optimal power flow (LWOPF) is developed and solved based on successive linear programming (SLP).

In Chapter 5, a nonlinear formulation for polar line-wise optimal power flow is developed and solved using classical nonlinear optimization technique considering voltage stability constraints. Chapter 6 shows the establishment of two developed linear models of incorporating the voltage stability in line-wise OPF. The solution algorithm for the developed two models using the SLP technique is presented and results are compared with exiting work to show the superior performance. Finally, the conclusions and suggestions for future work are drawn in Chapter 7. 


\begin{tabular}{|c|c|c|}
\hline $\begin{array}{l}\text { Power } \\
\text { Flow }\end{array}$ & $\rightarrow$ & Chapter 2: Polar Line-wise Power Flow (LWPF) Formulation \\
\hline$\downarrow$ & & \\
\hline \multirow{3}{*}{$\begin{array}{c}\text { Optimal } \\
\text { Power } \\
\text { Flow }\end{array}$} & $\rightarrow$ & Chapter 4: Line-wise Optimal Power Flow (LWOPF) \\
\hline & $\rightarrow$ & $\begin{array}{l}\text { Chapter 5: Nonlinear Optimization of Voltage Stability constrained OPF (VSC- } \\
\text { LWOPF) }\end{array}$ \\
\hline & $\rightarrow$ & $\begin{array}{l}\text { Chapter 6: Successive Linear Optimization of Voltage Stability constrained OPF } \\
\text { (VSC-LWOPF) }\end{array}$ \\
\hline
\end{tabular}

Figure 1.1 Structure of the thesis research work 


\section{Chapter Two}

\section{Chapter 2: Polar Line-Wise Power Flow and Voltage Collapse}

Voltage collapse (VC) is a phenomenon where voltage collapses at one or more busses due to lack of reactive power, characterized by a decrease in voltage with an increase in reactive power injection and voltage drop in connected lines due to large power flows. Bus-wise power balance equations, solved using NewtonRaphson (NR) technique, are widely used to analyze power systems for VC. While a bus-wise power balance equation succinctly models a power system, it and its Jacobian do not readily point out the most critical set of lines without additional analysis.

In this chapter, a line-wise set of equations for modeling a power system and its solution method using NR technique for power flow analysis are developed. Study results on 6-, 14-, 57- and 118-bus IEEE systems, a 582-bus real system, a 2383-bus Polish power system, and a 9241-bus PEGASE system show that the developed method is accurate, provides monotonic convergence, scales well for large systems and is consistently faster, up to twice the speed of the bus-wise NR method, while using sparse matrices.

Secondly, a line-wise VC Index is derived and shown to be directly present in the Jacobian of the linewise NR method, identifying the susceptible set of lines without additional computation. The usefulness of the VC index as an online VC assessment tool is demonstrated for a critical loading condition on test systems.

\subsection{Introduction}

Power flow (PF) analysis refers to the solution of a set of equations that describe a power system, yielding a bus-wise voltage solution for the whole power system. Power flows in transmission branches are computed using their impedance values and computed voltage solution. PF analysis plays a vital role in many aspects of electrical power systems engineering. PF analysis constitutes the basis for studies in fundamental topics such as Power Systems Optimization, Power Systems Planning, and Voltage Stability. The most popular formulation for PF analysis considers a set of bus-wise power balance equations based on modal analysis, expressed in polar and rectangular forms. The formulation is solved using numerical techniques such as Gauss-Seidel, Newton-Raphson (NR), and their variants such as the fast-decoupled PF. 
However, the bus-wise NR method and the associated Jacobian matrix cannot readily (i.e., without additional computation) point out the critical line, or the set of critical lines, that connect busses that are the most susceptible to voltage collapse. This inability to directly identify the critical line or the set of critical

lines that connect busses that are the most susceptible to voltage collapse remains a distinct shortcoming of voltage collapse assessment methods that rely on the bus-wise NR method. The proposed line-wise NR method does not suffer from this drawback as its Jacobian has terms that directly identify which set of critical lines connect busses that are the most susceptible to VC.

In the first part of this chapter, a line-wise NR method for PF analysis is proposed. The formulation comprising a set of line-wise power balance equations is presented. The set of equations is formulated in terms of the square of magnitude of bus voltages. It is solved using the NR technique. The solution directly yields bus-wise voltage solution and line-wise real and reactive power flows. The formulation easily scales for larger systems, demonstrates numerical stability, lends itself to sparse matrix techniques, provides monotonic convergence and consistently converges at a faster speed, up to twice the speed in comparison to the bus-wise NR method for PF analysis.

In the second part of the chapter, the voltage collapse index (VCI) that indicates the set of critical lines that connect busses that are the most susceptible to voltage collapse is developed. Thereafter, it is shown that VCI terms for all lines in the system are directly present in the Jacobian of the line-wise NR method. Hence, on completing PF analysis using the line-wise NR method, without additional computation, the critical line or the set of critical lines that connect the busses that are the most susceptible to voltage collapse can be directly identified. Analysis on IEEE 14, and 118-bus systems and a 582-bus real power system are completed, and results conclusively demonstrate the efficacy of the proposed line-wise NR method as a voltage collapse assessment tool. With the high computational speed of the line-wise NR method and its ability to directly identify the set of critical lines that connect the busses that are the most susceptible to VC, its use as an online $\mathrm{VC}$ assessment tool is proposed.

\subsection{Developed polar line-wise PF system of equations}

In this section, the developed formulation of the set of line-wise power balance equations is presented. Considering only the series impedance element of the pi-model of a transmission line or a transformer between buses $a$ and $b$, the circuit model is set out in Figure 2.1 Shunt elements of the pi-model are considered subsequently in (2.17) and (2.18), in the terms GS and BS. 


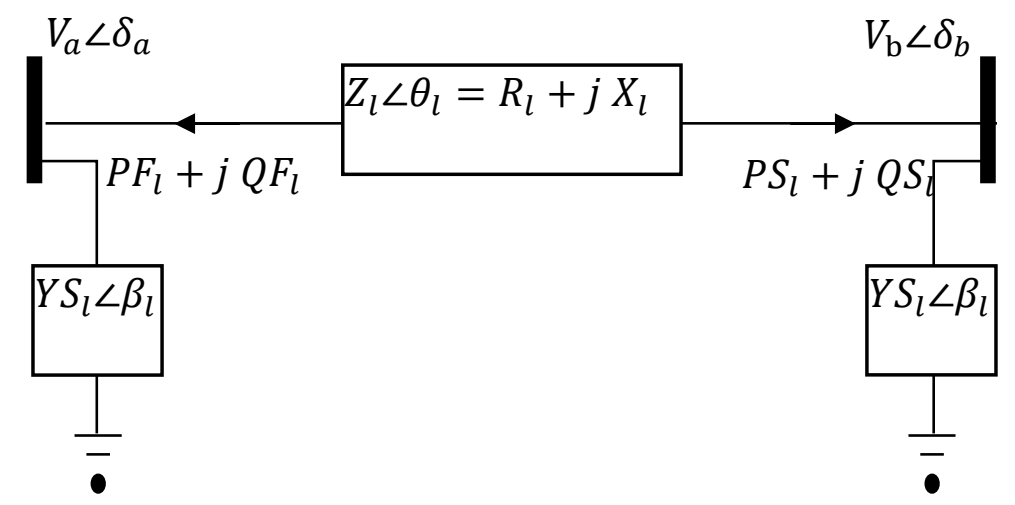

Figure 2.1 Series impedance element of the pi-model of the lth transmission branch connecting buses a and $\mathrm{b}$.

Based on [134], using the voltage difference between busses $a$ and $b$, power flow in the first end can be expressed as:

$$
V_{a} \angle \delta_{a}-V_{b} \angle \delta_{b}=\left(\frac{S S_{l} \angle \emptyset S_{l}}{V_{b} \angle \delta_{b}}\right)^{*} \cdot Z_{l} \angle \theta_{l}
$$

Multiplying both sides of (2.1) by the conjugate of $V_{b} \angle \delta_{b}$ :

$$
V_{a} \angle \delta_{a} \cdot\left(V_{b} \angle \delta_{b}\right)^{*}-V_{b}^{2}=\left(S S_{l} \angle \emptyset S_{l}\right)^{*} \cdot Z_{l} \angle \theta_{l}
$$

Separating the real and imaginary parts in (2.2)

$$
\begin{gathered}
V_{a} \cdot V_{\mathrm{b}} \cdot \cos \left(\delta_{a}-\delta_{b}\right)=P S_{l} \cdot R_{l}+Q S_{l} \cdot X_{l}+V_{b}^{2} \\
V_{a} \cdot V_{\mathrm{b}} \cdot \sin \left(\delta_{a}-\delta_{b}\right)=P S_{l} \cdot X_{l}-Q S_{l} \cdot R_{l}
\end{gathered}
$$

Dividing (2.4) by (2.3)

$$
\left(P S_{l} \cdot R_{l}+Q S_{l} \cdot X_{l}+V_{b}^{2}\right) \cdot \tan \left(\delta_{a}-\delta_{b}\right)=P S_{l} \cdot X_{l}-Q S_{l} \cdot R_{l}
$$

Summing the squares of (2.3) and (2.4), and rearranging yields, 


$$
V_{b}{ }^{4}+2 \cdot V_{b}{ }^{2}\left(P S_{l} \cdot R_{l}+Q S_{l} \cdot X_{l}-\frac{V_{a}^{2}}{2}\right)+S S_{l}{ }^{2} \cdot Z_{l}^{2}=0
$$

Similarly, for the second end:

$$
V_{b} \angle \delta_{b}-V_{a} \angle \delta_{a}=\left(\frac{S F_{l} \angle \emptyset F_{l}}{V_{a} \angle \delta_{a}}\right)^{*} Z
$$

Multiplying both sides by the conjugate of $V_{a} \angle \delta_{a}$ yields:

$$
V_{b} \angle \delta_{b} \cdot\left(V_{a} \angle \delta_{a}\right)^{*}-V_{a}^{2}=\left(S F_{l} \angle \emptyset F_{l}\right) \cdot Z_{l} \angle \theta_{l}
$$

Separating the real and imaginary parts in $(2.8)$

$$
\begin{gathered}
V_{b} \cdot V_{\mathrm{a}} \cdot \cos \left(\delta_{b}-\delta_{a}\right)=P F_{l} \cdot R_{l}+Q F_{l} \cdot X_{l}+V_{a}^{2} \\
V_{b} \cdot V_{\mathrm{a}} \cdot \sin \left(\delta_{b}-\delta_{a}\right)=P F_{l} \cdot X_{l}-Q F_{l} \cdot R_{l}
\end{gathered}
$$

Dividing (2.10) by (2.9)

$$
\left(P F_{l} \cdot R_{l}+Q F_{l} \cdot X_{l}+V_{a}^{2}\right) \cdot \tan \left(\delta_{b}-\delta_{a}\right)=P F_{l} \cdot X_{l}-Q F_{l} \cdot R_{l}
$$

Summing the squares of (2.9) and (2.10) and rearranging gets:

$$
V_{a}^{4}+2 V_{a}^{2}\left(P F_{l} \cdot R_{l}+Q F_{l} \cdot X_{l}-\frac{V_{b}^{2}}{2}\right)+S F_{l}^{2} \cdot Z_{l}^{2}=0
$$

Defining a matrix $[M]$, with a dimension of $N B \times 2 \cdot N T$, it is constructed as below by using the concept of the bus incidence matrix 


$$
\begin{array}{ll}
{[M]_{a, l}=1} & \text { if bus } a \text { is the first bus for line } l \\
{[M]_{b, N T+l}=1} & \text { if bus } b \text { is the second bus for line } l \\
{[M]_{a, l}=0} & \text { otherwise }
\end{array}
$$

Using $U$ instead of $V$ and collating (2.5), (2.6), (2.11) and (2.12) to model the entire transmission system, the following equations can be written as below. Consider a power system with NG generator buses and NLB load buses, such that the total number of buses $N B=N G+N L B$ and NT is the total number of transmission branches. The set of line-wise equations is defined below.

Using (2.12): $F F_{l}=$

$$
U_{a}^{2}+2 U_{a} \cdot\left(P F_{l} \cdot R_{l}+Q F_{l} \cdot X_{l}-\frac{U_{b}}{2}\right)+S F_{l}^{2} \cdot Z_{l}^{2}=0 \quad \forall l=1 \text { to } N T
$$

Using (2.6): $F S_{l}=$

$$
U_{b}{ }^{2}+2 U_{b}\left(P S_{l} \cdot R_{l}+Q S_{l} \cdot X_{l}-\frac{U_{a}}{2}\right)+S S_{l}^{2} \cdot Z_{l}^{2}=0 \quad \forall l=1 \text { to } N T
$$

Using (2.11): $F A_{l}=$

$$
\left(P F_{l} \cdot R_{l}+Q F_{l} \cdot X_{l}+U_{a}\right) \cdot \tan \left(\delta_{b}-\delta_{a}\right)-P F_{l} \cdot X_{l}+Q F_{l} \cdot R_{l}=0 \quad \forall l=1 \text { to } N T
$$

Using (2.5): $F B_{l}=$

$$
\left(P S_{l} \cdot R_{l}+Q S_{l} \cdot X_{l}+U_{b}\right) \cdot \tan \left(\delta_{a}-\delta_{b}\right)-P S_{l} \cdot X_{l}+Q S_{l} \cdot R_{l}=0 \quad \forall l=1 \text { to } N T
$$

Further, the bus-wise power balance equations can be written in a matrix form as below.

$$
\begin{aligned}
& F P=[M]\left[\begin{array}{l}
P F \\
P S
\end{array}\right]-U \cdot G S=P D-P G \\
& F Q=[M]\left[\begin{array}{l}
Q F \\
Q S
\end{array}\right]+U \cdot B S=Q D-Q G
\end{aligned}
$$


where the following is noted

(1) Shunt elements of the pi-model of transmission lines and transformers are included into $G S$ and $B S$, vectors of bus-wise conductance and susceptance elements, in (2.17) and (2.18).

(2) Constant power loads are modeled into $P D$ and $Q D$ of (2.17) and (2.18).

(3) Constant impedance loads, if any, are included into GS and BS, vectors of bus-wise conductance and susceptance elements, in (2.17) and (2.18).

The set of equations (2.13) - (2.18) models a power system. The total number of variables in this set of equations is $(4 \cdot N T+2 \cdot N B)$ and it equals the number equations. While $(2.13)-(2.16)$ have been developed in [134], the set of equations for line-wise power balance equations $(2.13)-(2.18)$ representing a power system is developed in this thesis. Defining the solution vector $w=[U \delta$ PF PS QF QS], the set of equations may be compactly presented as below:

$$
\begin{aligned}
& {\left[\begin{array}{l}
F F(w) \\
F S(w) \\
F A(w) \\
F B(w) \\
F P(w) \\
F Q(w)
\end{array}\right]=\left[\begin{array}{c}
0 \\
0 \\
0 \\
0 \\
{[P D-P G]} \\
{[Q D-Q G]}
\end{array}\right]} \\
& F T(w)=S T
\end{aligned}
$$

This set of nonlinear equations can be easily solved using Newton-Raphson technique. The following sections provide a solution method.

\subsection{Polar-form Line-Wise Power Flow Algorithm}

The system of equations (2.19) can be solved to determine the voltage solution using NR technique. This section describes the proposed algorithm to solve a set of equations that describe a power system using a multivariate NR technique. The method readily scales for larger systems and demonstrates a stable performance. Figure 2.2 shows the flowchart of the developed technique. It should be noted that the solution steps are similar to the standard bus-wise NR method for PF analysis, except that the set of equations to be 
solved is the set of line-wise power balance equations. In addition, there is no need to construct the bus admittance matrix for the line-wise NR method, which is a requirement for the bus-wise NR method.

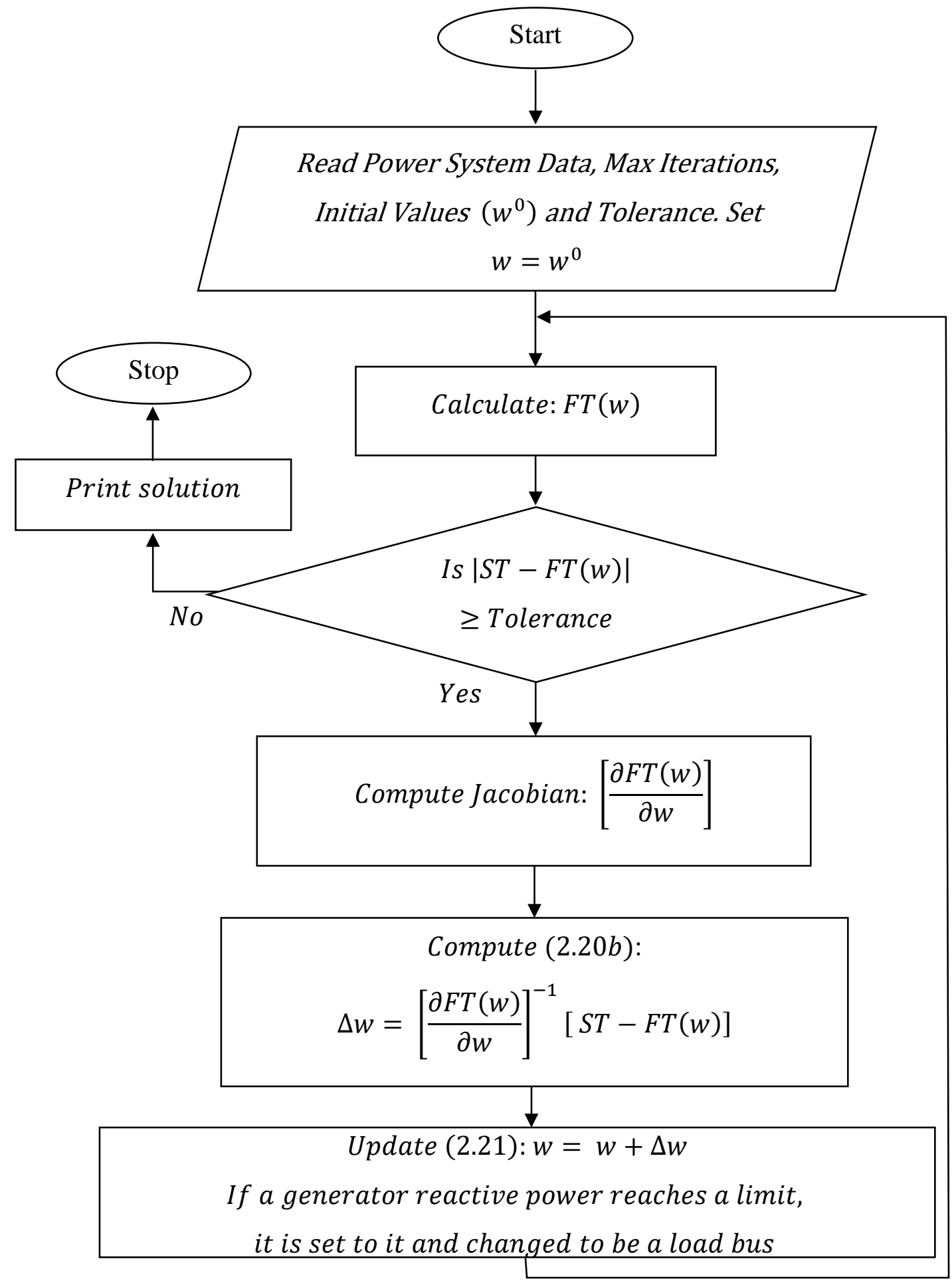

Figure 2.2 Flowchart of Polar-form Line-Wise Power Flow algorithm. 
Using Taylor's series expansion and limiting to the first order derivative while considering a solution $w,(2.19)$ can be expanded as below:

$$
\begin{gathered}
{\left[\begin{array}{l}
F F(w) \\
F S(w) \\
F A(w) \\
F B(w) \\
F P(w) \\
F Q(w)
\end{array}\right]+\left[\begin{array}{l}
\partial F F(w) / \partial w \\
\partial F S(w) / \partial w \\
\partial F A(w) / \partial w \\
\partial F B(w) / \partial w \\
\partial F P(w) / \partial w \\
\partial F Q(w) / \partial w
\end{array}\right] \cdot[\Delta w]=\left[\begin{array}{c}
0 \\
0 \\
0 \\
0 \\
{[P D-P G]} \\
{[Q D-Q G]}
\end{array}\right]} \\
\text { or } \\
F T(w)+\left[\frac{\partial F T(w)}{\partial w}\right] \cdot \Delta w=S T
\end{gathered}
$$

where $\left[\frac{\partial F T(w)}{\partial w}\right]$ is the Jacobian of the set of equations (2.13)- (2.18). In case $w$ does not satisfy the set of equations (2.19), then using (2.20a), an update for $w$ can be computed as below:

$$
\begin{gathered}
\Delta w=\left[\frac{\partial F T(w)}{\partial w}\right]^{-1} \cdot[S T-F T(w)] \quad \text { or } \\
\Delta w=\left[\frac{\partial F T(w)}{\partial w}\right]^{-1} \cdot[\Delta F T(w)]
\end{gathered}
$$

where $\Delta F T=[\Delta F F \Delta F S \Delta F A \triangle F B \Delta F P \Delta F Q]^{T} \quad$ and $\quad \Delta w=[\Delta U \Delta \delta \Delta \mathrm{PF} \triangle \mathrm{PS} \Delta \mathrm{QF} \Delta \mathrm{QS}]$. On computing $\Delta w$, the solution vector can be updated as:

$$
w=w+\Delta w
$$

By repeating the computation of $(2.20 \mathrm{~b})$ and (2.21) until the absolute value of all terms within $[S T-F T(w)]$ reduce to a zero or below an acceptably small value, the solution vector $w$ for the set of equations (2.19) is obtained.

It must be noted that the following are known:

(1) voltage magnitude and phase angle at the slack bus,

(2) real power injections (generation minus load) and bus voltage magnitudes at all generation busses, and, 
(3) real and reactive power injections (negative of real and reactive power loads) at load busses.

The unknowns then are:

(1) real and reactive power injections at the slack bus,

(2) bus voltage phase angles and reactive power injections at generator busses, and,

(3) voltage magnitudes and phase angles at load busses.

Knowns and unknowns are summarized in a tabular form below:

\begin{tabular}{c|c|c}
\hline & Knowns & Unknowns \\
\hline Slack Bus & $V$ & $P T=P G-P D$ \\
& $\delta$ & $Q T=Q G-Q D$ \\
\hline Generator Bus & $P T=P G-P D$ & $Q T=Q G-Q D$ \\
& $V$ & $\delta$ \\
\hline Load Bus & $P T=-P D$ & $V$ \\
& $Q T=-Q D$ & $\delta$ \\
\hline
\end{tabular}

Hence, given that real power injection is unknown for the slack bus, (2.17), (i.e., $F P(w)=P D-P G)$, must be solved for all busses except the slack bus. Similarly, given that reactive power injections are unknown for the all generator busses (including the slack bus), (2.18), (i.e., $F Q(w)=Q D-Q G)$, must be solved only for load busses.

Equations (2.13) to (2.16) for $F F(w), F S(w), F A(w)$, and $F B(w)$ have $N T$ equations each and all of them must be solved. Hence, the number of equations to be solved and unknowns are as below:

\begin{tabular}{c|c}
\hline Equations & Number \\
\hline$F F(w)$ & $N T$ \\
\hline$F S(w)$ & $N T$ \\
\hline$F A(w)$ & $N T$ \\
\hline$F B(w)$ & $N T$ \\
\hline$F P(w)$ & $N B-1$ \\
\hline$F Q(w)$ & $N L B$ \\
\hline
\end{tabular}

\begin{tabular}{c|c}
\hline Unknowns & Number \\
\hline$P F$ & $N T$ \\
\hline$P S$ & $N T$ \\
\hline$Q F$ & $N T$ \\
\hline$Q S$ & $N T$ \\
\hline$\delta$ & $N B-1$ \\
\hline$V$ & $N L B$ \\
\hline
\end{tabular}


The total number of equations is $(4 \cdot N T+N B-1+N L B)$. The total number of unknowns is $(4 \cdot N T+N B-1+N L B)$. The result of the developed algorithm will be as follows:

(1) the squares of voltage magnitudes at load busses,

(2) voltage phase angles at all busses except the slack bus, and,

(3) real and reactive powers on both ends for all branches (lines and transformers).

Details of the Jacobian of the set of equations $(2.13)-(2.18),\left[\frac{\partial F T(w)}{\partial w}\right]$, are provided in the following section.

\subsection{Development of the Jacobian Matrix}

This section presents the mathematical development of the Jacobian matrix, which is the partial differentiation for the set of equations (2.13) - (2.18), with respect to the six unknown vectors $\left[\begin{array}{lllll}U & \delta & P F & P S & Q F\end{array}\right]$. The complete system Jacobian is presented below considering (2.20b). The size of the submatrices of the system Jacobian is also documented below:

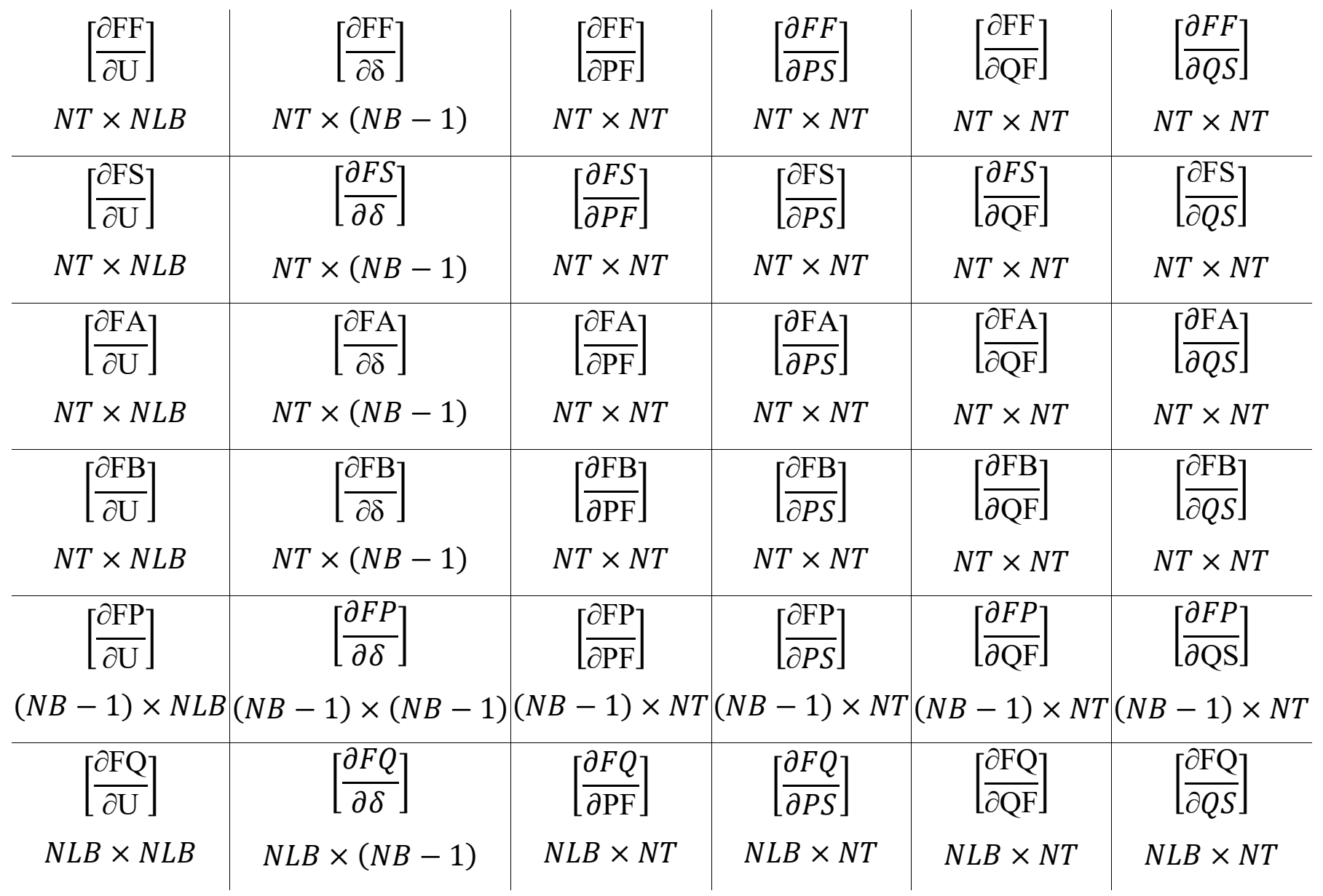


The following are null submatrices in the Jacobian:

$\left[\frac{\partial \mathrm{FF}}{\partial \delta}\right],\left[\frac{\partial \mathrm{FF}}{\partial \mathrm{PS}}\right],\left[\frac{\partial \mathrm{FF}}{\partial \mathrm{QS}}\right],\left[\frac{\partial F S}{\partial \delta}\right],\left[\frac{\partial F S}{\partial P F}\right],\left[\frac{\partial \mathrm{FF}}{\partial \mathrm{QF}}\right],\left[\frac{\partial \mathrm{FA}}{\partial \mathrm{PS}}\right],\left[\frac{\partial \mathrm{FA}}{\partial \mathrm{QS}}\right],\left[\frac{\partial \mathrm{FB}}{\partial \mathrm{PF}}\right],\left[\frac{\partial \mathrm{FB}}{\partial \mathrm{QF}}\right],\left[\frac{\partial F P}{\partial \delta}\right],\left[\frac{\partial F P}{\partial \mathrm{QF}}\right],\left[\frac{\partial F P}{\partial Q S}\right],\left[\frac{\partial F Q}{\partial \delta}\right]$, $\left[\frac{\partial F Q}{\partial \mathrm{PF}}\right]$, and, $\left[\frac{\partial F Q}{\partial P S}\right]$.

The non-zero elements of partial differentiation of (2.13) with respect to $U, \delta, \mathrm{PF}, \mathrm{PS}, \mathrm{QF}, \mathrm{QS}$ for $l \in$ $\{1,2, \ldots, N T\}$ are as follows:

$$
\begin{array}{cc}
\frac{\partial F F_{l}}{\partial U_{a}}=2 \cdot U_{a}+2 \cdot\left(P F_{l} \cdot R_{l}+Q F_{l} \cdot X_{l}-\frac{U_{b}}{2}\right) & \forall\{a, b\} \in l \\
\frac{\partial F F_{l}}{\partial U_{b}}=-U_{a} & \forall\{a, b\} \in l \\
\frac{\partial F F_{l}}{\partial P F_{l}}=2\left(U_{a} \cdot R_{l}+P F_{l} \cdot Z_{l}^{2}\right) & \forall\{a, b\} \in l \\
\frac{\partial F F_{l}}{\partial Q F_{l}}=2\left(U_{a} \cdot X_{l}+Q F_{l} \cdot Z_{l}^{2}\right) & \\
& \forall\{a, b\} \in l
\end{array}
$$

The non-zero elements of partial differentiation of (2.14) with respect to $U, \delta, \mathrm{PF}, \mathrm{PS}, \mathrm{QF}, \mathrm{QS}$ for $l \in$ $\{1,2, \ldots, N T\}$ are as follows:

$$
\begin{array}{cc}
\frac{\partial F S_{l}}{\partial U_{a}}=-U_{b} & \forall\{a, b\} \in l \\
\frac{\partial F S_{l}}{\partial U_{b}}=2 \cdot U_{b}+2 \cdot\left(P S_{l} \cdot R_{l}+Q S_{l} \cdot X_{l}-\frac{U_{a}}{2}\right) & \forall\{a, b\} \in l \\
\frac{\partial F S_{l}}{\partial P S_{l}}=2\left(U_{b} \cdot R_{l}+P S_{l} \cdot Z_{l}^{2}\right) & \forall\{a, b\} \in l \\
\frac{\partial F S_{l}}{\partial Q S_{l}}=2\left(U_{b} \cdot X_{l}+Q S_{l} \cdot Z_{l}^{2}\right) & \forall\{a, b\} \in l
\end{array}
$$


The non-zero elements of partial differentiation of (2.15) with respect to $U, \delta, \mathrm{PF}, \mathrm{PS}, \mathrm{QF}, \mathrm{QS}$ for $\forall l \in$ $\{1,2, \ldots, N T\}$ are as follows:

$$
\begin{array}{ll}
\frac{\partial F A_{l}}{\partial U_{a}}=\tan \left(\delta_{b}-\delta_{a}\right) & \forall\{a, b\} \in l \\
\frac{\partial F A_{l}}{\partial \delta_{a}}=-\left(P F_{l} \cdot R_{l}+Q F_{l} \cdot X_{l}+U_{a}\right) \cdot \sec ^{2}\left(\delta_{b}-\delta_{a}\right) & \forall\{a, b\} \in l \\
\frac{\partial F A_{l}}{\partial \delta_{b}}=\left(P F_{l} \cdot R_{l}+Q F_{l} \cdot X_{l}+U_{a}\right) \cdot \sec ^{2}\left(\delta_{b}-\delta_{a}\right) & \forall\{a, b\} \in l \\
\frac{\partial F A_{l}}{\partial P F_{l}}=R_{l} \cdot \tan \left(\delta_{b}-\delta_{a}\right)-X_{l} & \\
\frac{\partial F A_{l}}{\partial Q F_{l}}=X_{l} \cdot \tan \left(\delta_{b}-\delta_{a}\right)+R_{l} & \forall\{a, b\} \in l
\end{array}
$$

The non-zero elements of partial differentiation of (2.16) with respect to $U, \delta, \mathrm{PF}, \mathrm{PS}, \mathrm{QF}, \mathrm{QS}$ for $\forall l \in$ $\{1,2, \ldots, N T\}$ are as follows:

$$
\begin{array}{cc}
\frac{\partial F B_{l}}{\partial U_{b}}=\tan \left(\delta_{a}-\delta_{b}\right) & \forall\{a, b\} \in l \\
\frac{\partial F B_{l}}{\partial \delta_{a}}=\left(P S_{l} \cdot R_{l}+Q S_{l} \cdot X_{l}+U_{b}\right) \cdot \sec ^{2}\left(\delta_{a}-\delta_{b}\right) & \forall\{a, b\} \in l \\
\frac{\partial F B_{l}}{\partial \delta_{b}}=-\left(P S_{l} \cdot R_{l}+Q S_{l} \cdot X_{l}+U_{b}\right) \cdot \sec ^{2}\left(\delta_{a}-\delta_{b}\right) & \forall\{a, b\} \in l \\
\frac{\partial F B_{l}}{\partial P S_{l}}=R_{l} \cdot \tan \left(\delta_{a}-\delta_{b}\right)-X_{l} & \\
\frac{\partial F B_{l}}{\partial Q S_{l}}=X_{l} \cdot \tan \left(\delta_{a}-\delta_{b}\right)+R_{l} & \forall\{a, b\} \in l \\
& \forall\{a, b\} \in l
\end{array}
$$


The non-zero elements of partial differentiation of (2.17) with respect to $U, \delta, \mathrm{PF}, \mathrm{PS}, \mathrm{QF}, \mathrm{QS}$ for $\forall a \in$ $\{1,2, \ldots, N B\}$ are below:

$$
\frac{\partial F P_{a}}{\partial U_{a}}=-G S_{a} \quad \forall a \in\{1,2, \ldots, N B\}
$$

And for $l \in\{1,2, \ldots, N T\}$ as below:

$$
\begin{array}{ll}
\frac{\partial F P_{a}}{\partial P F_{l}}=[M]_{a, l} & \forall\{a, b\} \in l \\
\frac{\partial F P_{b}}{\partial P S_{l}}=[M]_{b, N T+l} & \forall\{a, b\} \in l
\end{array}
$$

The non-zero elements of partial differentiation of (2.18) with respect to $U, \delta, \mathrm{PF}, \mathrm{PS}, \mathrm{QF}, \mathrm{QS}$ for $\forall a \in$ $\{1,2, \ldots, N B\}$ are below:

$$
\frac{\partial F Q_{a}}{\partial U_{a}}=B S_{a} \quad \forall\{a, b\} \in l
$$

And for $l \in\{1,2, \ldots, N T\}$ as below:

$$
\begin{array}{ll}
\frac{\partial F Q_{a}}{\partial Q F_{l}}=[M]_{a, l} & \forall\{a, b\} \in l \\
\frac{\partial F Q_{b}}{\partial Q S_{l}}=[M]_{b, N T+l} & \forall\{a, b\} \in l
\end{array}
$$

The Jacobian matrix of the proposed set of equations is a sparse matrix leading to a low computation burden. The overall dimension of the matrix is $(4 \cdot N T+N B-1+N L B) \times(4 \cdot N T+N B-1+N L B)$. Figure 2.3 shows the sparsity patterns of Jacobian matrix for a 6-bus. 


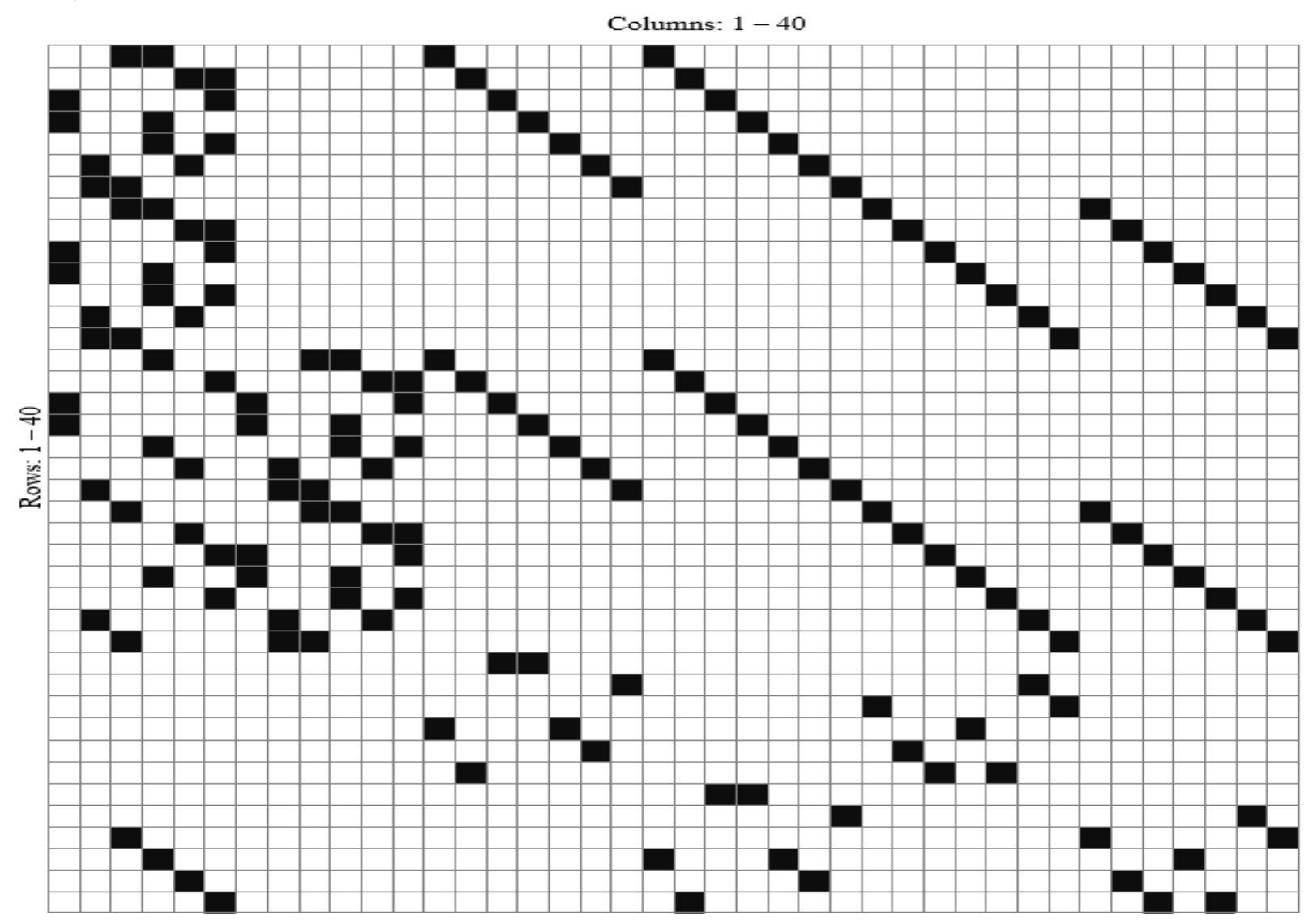

Figure 2.3 Plot of 6-bus System Jacobian with 40 rows and 40 columns.

\subsection{Voltage Collapse Index}

In this section, the VCI is derived which identifies the critical set of lines [41]. It will be shown that the Jacobian of the line-wise NR method directly provides VCI values identifying the critical set of lines without further computation.

The solution of (2.14), as a quadratic equation in $U_{b}$, yields the two roots given in (2.46), where the solution will physically exist if $U_{b}$ values are: (1) real and unequal before voltage collapse, and, (2) real and equal at voltage collapse.

$$
U_{b}=-\left(P S_{l} \cdot R_{l}+Q S_{l} \cdot X_{l}-\frac{U_{a}}{2}\right) \pm \sqrt{\left(P S_{l} \cdot R_{l}+Q S_{l} \cdot X_{l}-\frac{U_{a}}{2}\right)^{2}-S S_{l}{ }^{2} \cdot Z_{l}{ }^{2}}
$$


At the VC point, the two roots of $U_{b}$ are equal and the second term of (2.46) inside the square root reduces to a zero. Thus, at VC condition:

$$
U_{b}=-\left(P S_{l} \cdot R_{l}+Q S_{l} \cdot X_{l}-\frac{U_{a}}{2}\right) \quad \text { or } \quad U_{b}+\left(P S_{l} \cdot R_{l}+Q S_{l} \cdot X_{l}-\frac{U_{a}}{2}\right)=0
$$

The partial differentiation of $F S_{l}$ in (2.14) with respect to $U_{b}$ is given in (2.27) and is an element of $\left[\frac{\partial F S}{\partial U}\right]$ submatrix of the Jacobian of line-wise NR method. It can be seen that the VC condition in (2.47) appears as the right-hand side of (2.27). Hence, on VC this Jacobian element of the line-wise NR method corresponding to the submatrix $\left[\frac{\partial F S}{\partial U}\right]$ will reduce to a zero, identifying that the $l^{\text {th }}$ line suffers a VC.

Considering $F F_{l}$ in (2.13), at $\mathrm{VC}$, its solution for $U_{a}$ leads to:

$$
U_{a}=-\left(P F_{l} \cdot R_{l}+Q F_{l} \cdot X_{l}-\frac{U_{b}}{2}\right) \quad \text { or } \quad U_{a}+\left(P F_{l} \cdot R_{l}+Q F_{l} \cdot X_{l}-\frac{U_{b}}{2}\right)=0
$$

The partial differentiation of $F F_{l}$ in (2.13) with respect to $U_{a}$ is given in (2.22) and is an element of the $\left[\frac{\partial F F}{\partial U}\right]$ submatrix of the Jacobian of the line-wise NR method. It can be seen that the VC condition in (2.48) appears as the right-hand side of (2.22). Hence, on VC the Jacobian element of the line-wise NR method corresponding to the submatrix $\left[\frac{\partial F F}{\partial U}\right]$ will reduce to a zero, identifying that the $l^{\text {th }}$ line suffers a VC.

From the above, it can be seen that at the end of the line-wise NR method for PF, without any additional computation, the set of critical lines that connect busses that are the most susceptible to VC can be determined by examining Jacobian submatrices $\left[\frac{\partial F F}{\partial U}\right]$ and $\left[\frac{\partial F S}{\partial U}\right]$.

The right-hand sides of (2.22) and (2.27) are defined as VC indices $V C I_{a}$ and $V C I_{b}$ respectively:

$$
\begin{aligned}
& V C I_{a}=\frac{\partial F F_{l}}{\partial U_{a}}=2 \cdot U_{a}+2 \cdot\left(P F_{l} \cdot R_{l}+Q F_{l} \cdot X_{l}-\frac{U_{b}}{2}\right) \\
& V C I_{b}=\frac{\partial F S_{l}}{\partial U_{b}}=2 \cdot U_{b}+2 \cdot\left(P S_{l} \cdot R_{l}+Q S_{l} \cdot X_{l}-\frac{U_{a}}{2}\right)
\end{aligned}
$$


It is important to note that conditions (2.49) and (2.50) were reported in [32]; however, their direct existence in the Jacobian of line-wise NR method is reported here. On loading a power system, indices $V C I_{a}$ and $V C I_{b}$ reduce to a zero at $\mathrm{VC}$ as shown in the next section.

\subsection{Results of System Studies}

In this section, test results of the proposed line-wise NR method are reported and compared with those from the bus-wise NR method. The proposed algorithm is coded in MATLAB (version R2016a). The buswise NR method is taken from the MATPOWER software package [135]. All tests are done on a 64-bit i7 Intel Core laptop $(2.6 \mathrm{GHz}, 16 \mathrm{~GB}$ of RAM) with the Windows 10 operating system. Sparse matrix implementations are considered where possible.

Table 2.1 reports test results such as the number of iterations and the execution times for both linewise and bus-wise NR methods at a tolerance of $10^{-5}$ per-unit. It is seen that the proposed line-wise NR method is computationally equal or up to twice as fast as the bus-wise NR method.

Table 2.1 Number of Iterations and Execution Times at $10^{-5}$ per unit tolerance

\begin{tabular}{c|c|c|c|c|c}
\hline & \multicolumn{2}{|c|}{ Traditional } & \multicolumn{2}{c}{$\begin{array}{c}\text { Proposed method } \\
\text { (Line-wise NR) }\end{array}$} & $\begin{array}{c}\text { Execution time of } \\
\text { line-wise method as } \\
\text { bus-wise NR (MATPOWER) }\end{array}$ \\
\cline { 2 - 6 } & $\begin{array}{c}\text { Execution Time } \\
\text { (milliseconds) }\end{array}$ & $\begin{array}{c}\text { \# of } \\
\text { Iterations }\end{array}$ & $\begin{array}{c}\text { Execution Time } \\
\text { (milliseconds) }\end{array}$ & \# of Iterations & $\begin{array}{c}\text { Percentage of bus- } \\
\text { wise method }\end{array}$ \\
\hline 6-bus & 4.0 & 3 & 4.0 & 4 & $100.00 \%$ \\
\hline 14-bus & 9.0 & 2 & 4.0 & 3 & $44.44 \%$ \\
\hline 57-bus & 22.0 & 3 & 8.0 & 3 & $36.36 \%$ \\
\hline 118-bus & 31.0 & 3 & 15.0 & 6 & $48.39 \%$ \\
\hline 2383-bus & 332.0 & 6 & 152.0 & 4 & $59.28 \%$ \\
\hline 9241-bus & 1579.0 & 4 & 936.0 & 6 & $57.15 \%$ \\
\hline
\end{tabular}


Loads are modeled as constant MVA loads, and lines and transformers are modeled as pi-models considering their shunt admittance elements. Convergence tolerance is set to $10^{-5}$ per-unit.

To illustrate the quality of solution from the line-wise NR method, the differences in results between the bus-wise NR method and the line-wise NR method for bus voltage magnitudes and angles are determined and quantified as Root Mean Square Error (RMSE) values. These RMSE values are reported in Figure 2.4 for the 6,14,57, and 118-bus IEEE systems and the 582-bus real system. With very low RMSE values, the comparison shows that the proposed method has an accurate performance in terms of solution accuracy.

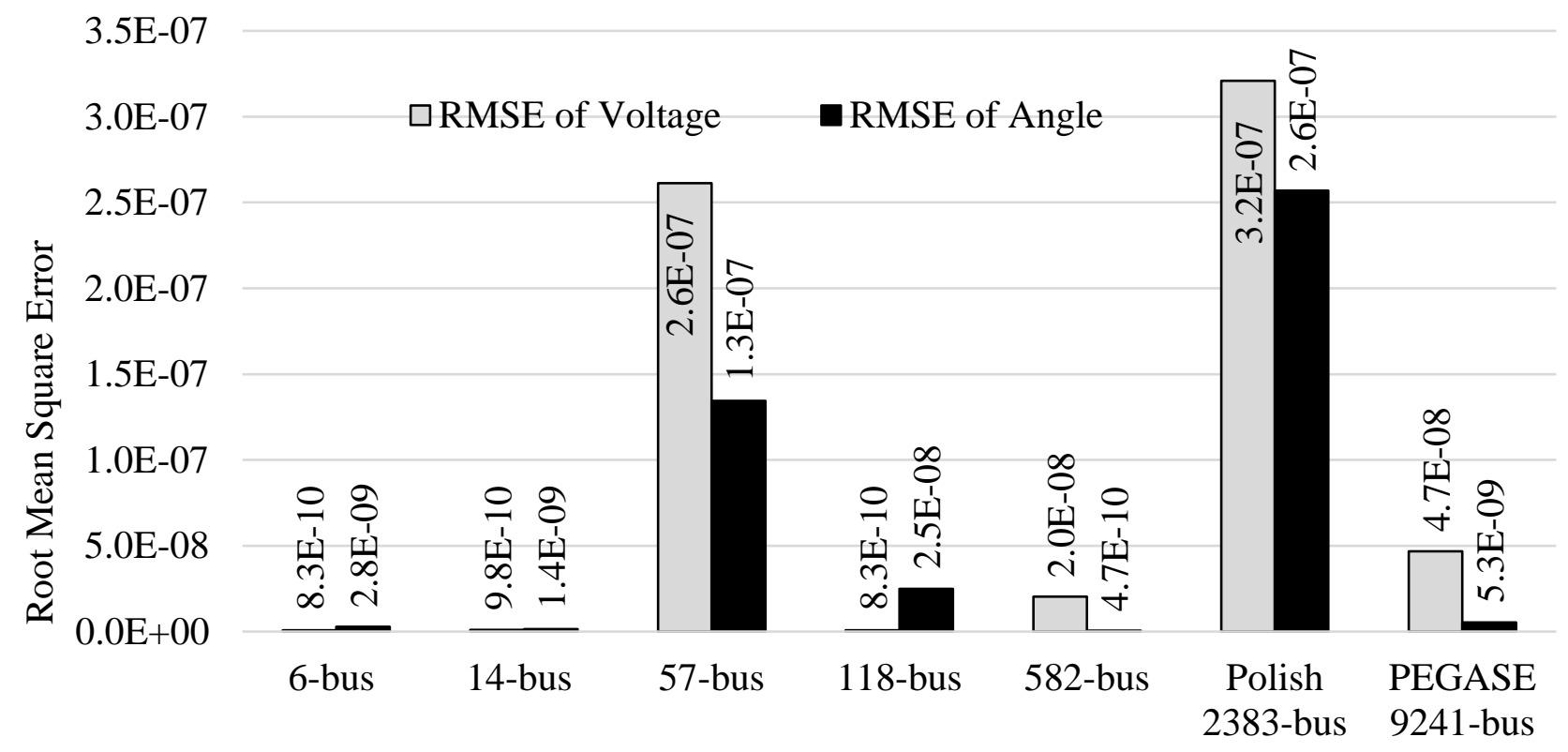

Figure 2.4 The Root Mean Square Error (RMSE) of bus voltage magnitudes and angles for difference in results between PF solutions of the traditional bus-wise NR method and the proposed line-wise NR method.

The convergence characteristics of the line-wise NR method for 6-, 14-, 57- and 118-bus IEEE systems, a 582-bus real system, 2383-bus Polish power system, and a 9241-bus PEGASE system are shown in Figure 2.5. The results establish that the proposed method shows consistent monotonic convergence properties for all the systems studied. 


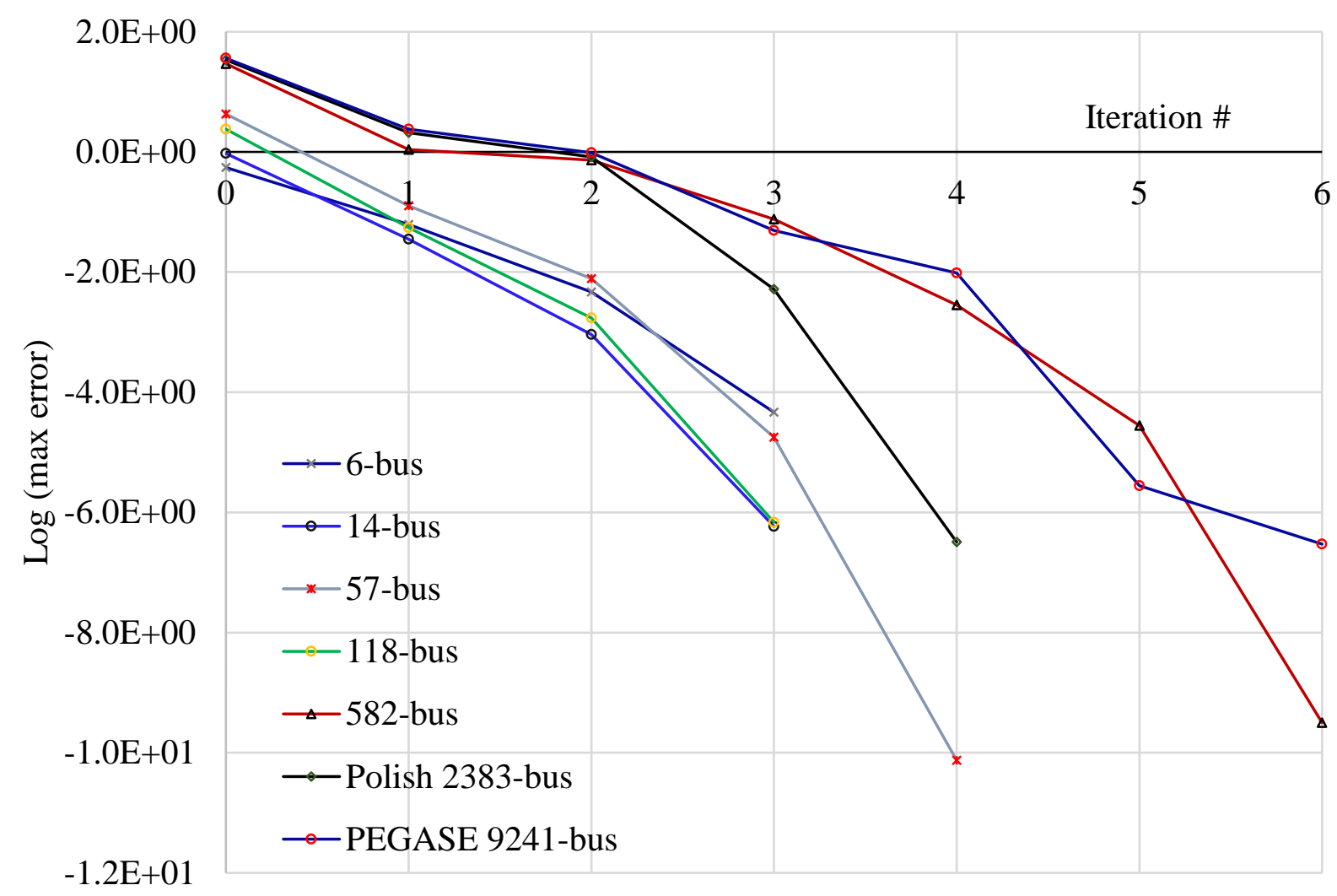

Figure 2.5 Convergence characteristic of the proposed method for different test systems. 


\subsection{Discussion on Power Flow}

The proposed line-wise NR method for PF is consistently equal or faster than bus-wise NR method, up to twice the speed. The reasons for the faster speed of the line-wise NR method are discussed here. The use of $U$, square of voltage magnitude in the formulation, instead of voltage magnitude by itself, makes power relations linear in $\mathrm{FP}(\mathrm{w})$ and $\mathrm{FQ}(\mathrm{w}),(2.17)$ and (2.18). In contrast, the bus-wise $\mathrm{PF}$ method uses voltage magnitudes, making power relations quadratic. To elaborate, a comparison is drawn between the number of Jacobian terms of the line-wise NR method and the bus-wise NR method in Table 2.2. The buswise NR method also has a lower number of products computed each time the mismatch vector is computed. That said, for the bus-wise method each of those products has a sine or a cosine function. Whereas, in the case of the line-wise NR method, only about $15 \%$ of the terms involve a cosine or a sine function.

Table 2.2 Comparison of Non-zero Terms of the Line-wise NR and Bus-wise NR method formulations

\begin{tabular}{|c|c|c|c|c|c|c|c|c|}
\hline & \multirow[b]{2}{*}{ Method } & \multirow[b]{2}{*}{$\begin{array}{c}\text { Number } \\
\text { Of } \\
\text { Equations }\end{array}$} & \multicolumn{4}{|c|}{ Number of Terms } & \multicolumn{2}{|c|}{ Number of Products } \\
\hline & & & $4^{\text {th }}$ Order & $3^{\text {rd }}$ Order & $2^{\text {nd }}$ Order & $1^{\text {st }}$ Order & $\begin{array}{c}\text { With Sine } \\
\text { or Cosine } \\
\text { Terms }\end{array}$ & $\begin{array}{c}\text { Without Sine } \\
\text { or Cosine } \\
\text { Terms }\end{array}$ \\
\hline \multirow{2}{*}{ 6-Bus } & Line-wise & 37 & 0 & 0 & 168 & 40 & 56 & 320 \\
\hline & Bus-wise & 9 & 44 & 9 & 0 & 0 & 203 & 0 \\
\hline \multirow{2}{*}{ 14-bus } & Line-wise & 102 & 0 & 0 & 480 & 108 & 160 & 908 \\
\hline & Bus-wise & 22 & 130 & 22 & 0 & 0 & 586 & 0 \\
\hline \multirow{2}{*}{ 57-bus } & Line-wise & 426 & 0 & 0 & 1,920 & 434 & 640 & 3,634 \\
\hline & Bus-wise & 106 & 562 & 106 & 0 & 0 & 2,566 & 0 \\
\hline \multirow{2}{*}{ 118-bus } & Line-wise & 925 & 0 & 0 & 4,464 & 980 & 1,488 & 8,420 \\
\hline & Bus-wise & 181 & 1,034 & 181 & 0 & 0 & 4,679 & 0 \\
\hline \multirow{2}{*}{ 582-bus } & Line-wise & 4,878 & 0 & 0 & 23,160 & 5,024 & 7,720 & 43,624 \\
\hline & Bus-wise & 1,018 & 5,140 & 1,018 & 0 & 0 & 23,614 & 0 \\
\hline \multirow{2}{*}{ 2383-bus } & Line-wise & 16,022 & 0 & 0 & 69,504 & 16,350 & 23,168 & 132,190 \\
\hline & Bus-wise & 4,438 & 22,408 & 4,438 & 0 & 0 & 102,946 & 0 \\
\hline \multirow{2}{*}{ 9241-bus } & Line-wise & 81,232 & 0 & 0 & 385,176 & 82,678 & 128,392 & 724,638 \\
\hline & Bus-wise & 17,036 & 86,017 & 17,036 & 0 & 0 & 395,175 & 0 \\
\hline
\end{tabular}


Table 2.3 Comparison of Jacobian Computations of Line-wise and Bus-wise PF methods using Newton Raphson technique for Non-zero Terms

\begin{tabular}{|c|c|c|c|c|c|c|c|}
\hline & Method & $\begin{array}{c}\text { Number } \\
\text { of } \\
\text { Jacobian } \\
\text { Elements }\end{array}$ & \begin{tabular}{|c} 
Number \\
of \\
Fourth Order \\
Terms
\end{tabular} & $\begin{array}{c}\text { Number } \\
\text { of } \\
\text { Cubic } \\
\text { Terms }\end{array}$ & $\begin{array}{c}\text { Number } \\
\text { of } \\
\text { Quadratic } \\
\text { Terms }\end{array}$ & $\begin{array}{l}\text { Number } \\
\text { of } \\
\text { Linear } \\
\text { Terms }\end{array}$ & $\begin{array}{c}\text { Number of } \\
\text { Products }\end{array}$ \\
\hline \multirow{2}{*}{ 6- Bus } & Line-wise & 136 & 0 & 0 & 56 & 336 & 448 \\
\hline & Bus-wise & 49 & 145 & 17 & 0 & 0 & 631 \\
\hline \multirow{2}{*}{ 14-bus } & Line-wise & 381 & 0 & 0 & 160 & 960 & 1,280 \\
\hline & Bus-wise & 146 & 464 & 40 & 0 & 0 & 1,976 \\
\hline \multirow{2}{*}{ 57-bus } & Line-wise & 1,678 & 0 & 0 & 640 & 3840 & 5,120 \\
\hline & Bus-wise & 718 & 2,254 & 206 & 0 & 0 & 9,634 \\
\hline \multirow{2}{*}{$\begin{array}{l}118- \\
\text { bus }\end{array}$} & Line-wise & 3,310 & 0 & 0 & 1488 & 8928 & 11,904 \\
\hline & Bus-wise & 1,049 & 3,269 & 309 & 0 & 0 & 14,003 \\
\hline \multirow{2}{*}{$\begin{array}{l}582- \\
\text { bus }\end{array}$} & Line-wise & 20,916 & 0 & 0 & 7720 & 46320 & 61,760 \\
\hline & Bus-wise & 6,740 & 21,284 & 1,892 & 0 & 0 & 90,812 \\
\hline \multirow{2}{*}{$\begin{array}{c}\text { 2383- } \\
\text { bus }\end{array}$} & Line-wise & 56,393 & 0 & 0 & 23,168 & 139,008 & 185,344 \\
\hline & Bus-wise & 27,783 & 87,147 & 7,747 & 0 & 0 & 371,830 \\
\hline \multirow{2}{*}{$\begin{array}{c}9241- \\
\text { bus }\end{array}$} & Line-wise & 332,302 & 0 & 0 & 128,392 & 770,352 & $1,027,136$ \\
\hline & Bus-wise & 128,689 & 337,947 & 30,041 & 0 & 0 & $1,441,914$ \\
\hline
\end{tabular}

On reviewing Table 2.1, the number of products required to compute a Jacobian in the bus-wise NR method is about 1.5 times (ranging from the lowest of 1.15 times to the highest of 1.88 times) that of linewise NR method. Further, except for the diagonal terms, every term of Jacobian for the bus-wise NR method requires computation of a sine or a cosine function. However, for the line-wise NR method, only Jacobian terms corresponding to equations $\mathrm{FA}(\mathrm{w})$ and $\mathrm{FB}(\mathrm{w})$, (i.e. (2.15) and (2.16)), have cosine or sine functions. These aspects make the computation of Jacobian terms for the line-wise NR method much faster in comparison with the bus-wise NR method. In an iterative algorithm, such as the PF analysis, these differences add up and make the line-wise NR method appreciably faster than the bus-wise NR method. 
Further, to compare the characteristics of the Jacobian matrices of the line-wise NR method and the bus-wise NR method, the Condition number and Sparsity factors are reported in Table 2.4. For the line-wise NR method, the dimension of the Jacobian matrix is computed using details provided at the end of section 2.4, where the total number of equations and unknowns equal $(4 \cdot N T+N B-1+N L B)$. Condition numbers of line-wise NR and bus-wise NR methods are well-behaved. Sparsity factors also remain similar.

Table 2.4 Comparison of Size and Condition Number of Jacobian Matrix for Both Line-wise NR and Buswise NR methods

\begin{tabular}{c|c|c|c|c|c|c}
\hline \multirow{2}{*}{$\begin{array}{c}\text { Number of } \\
\text { Busses }\end{array}$} & \multicolumn{2}{|c|}{ Traditional bus-wise Jacobian matrix } & \multicolumn{3}{c}{ Proposed line-wise Jacobian matrix } \\
\cline { 2 - 7 } & Size & $\begin{array}{c}\text { Condition } \\
\text { Number }\end{array}$ & $\begin{array}{c}\text { Sparsity } \\
\text { Factor }\end{array}$ & Size & $\begin{array}{c}\text { Condition } \\
\text { Number }\end{array}$ & $\begin{array}{c}\text { Sparsity } \\
\text { Factor }\end{array}$ \\
\hline 6 & $9 \times 9$ & $5.3 \cdot 10^{1}$ & 39.50 & $37 X 37$ & $3.4 \cdot 10^{2}$ & 90.10 \\
\hline 14 & $22 \times 22$ & $2.4 \cdot 10^{2}$ & 69.80 & $102 X 102$ & $1.9 \cdot 10^{3}$ & 96.30 \\
\hline 57 & $106 \times 106$ & $2.9 \cdot 10^{3}$ & 93.60 & $422 X 422$ & $1.8 \cdot 10^{4}$ & 99.10 \\
\hline 118 & $181 X 181$ & $5.7 \cdot 10^{3}$ & 96.80 & $925 X 925$ & $9.0 \cdot 10^{4}$ & 99.60 \\
\hline 582 & $1018 X 1018$ & $6.5 \cdot 10^{6}$ & 99.30 & $4878 X 4878$ & $7.1 \cdot 10^{7}$ & 99.90 \\
\hline 9241 & $17036 \times 17036$ & $1.6 \cdot 10^{7}$ & 99.96 & $81232 X 81232$ & $6.8 \cdot 10^{8}$ & 99.99 \\
\hline
\end{tabular}

\subsection{Benefits of the Proposed Method}

To envisage the benefits of using the developed polar LWPF on real-time power system operation, a numerical example for the province of Ontario is provided. The Independent Energy System Operator (IESO) schedules Ontario's power system a day-ahead and provides the optimal hourly power dispatch schedule for all generators. Each of these 24 sets of optimal generation schedules should be checked for transmission system feasibility by conducting N-1 contingency analysis, for each hour, considering that $\mathrm{N}$ elements comprise the power system. An N-1 contingency analysis requires N power flow solutions, one each considering an outage of one of the $\mathrm{N}$ elements. Considering Ontario's power system comprising 10,000 elements, PF analysis should be conducted 10,000 times for each hour and 240,000 times for a day. As can be seen in Table 2.1, a 10,000-element power system shall take about four (4) seconds per power flow analysis using BWPF and two (2) seconds using LWPF. Therefore, for Ontario's power system 
comprising 10,000 elements, BWPF will take 960,000 seconds (266 hours) for N-1 contingency analysis considering a 24-hour schedule, whereas LWPF will only take 480,000 seconds (133 hours). Thus, using LWPF instead of BWPF will enable a system operator such as the IESO to assess the health of the transmission system in a reduced time, facilitating the initiation of remedial actions in reduced time where required, thereby reinforcing operation of the power system in the most safe and stable manner. Finally, it can be discerned that the line-wise NR method scales very well for larger systems without any difficulty and hence is suitable for commercial-grade applications.

\subsection{Performance of Voltage Collapse Index}

In this section, the performance of indices $V C I_{a}$ and $V C I_{b}$ are reported for the 14-bus and 118-bus IEEE systems and for a 582-bus real system for brevity. As the load modeling is very important for VC analysis, the proposed study considers the constant power (constant MVA) model, which is the most conservative load model for voltage stability studies [29, 136].

Figure 2.6 shows the values of VCI taken directly from the Jacobian of the line-wise NR method for all the twenty lines at both ends of the IEEE 14-bus system at VC when bus loads are scaled by 3.83 times. It shows that the fifth line is the most critical as it connects busses that are susceptible to VC, where $V C I_{a}$ and $V C I_{b}$ values are $4.70 \mathrm{E}-01$ and 0.0 respectively. 


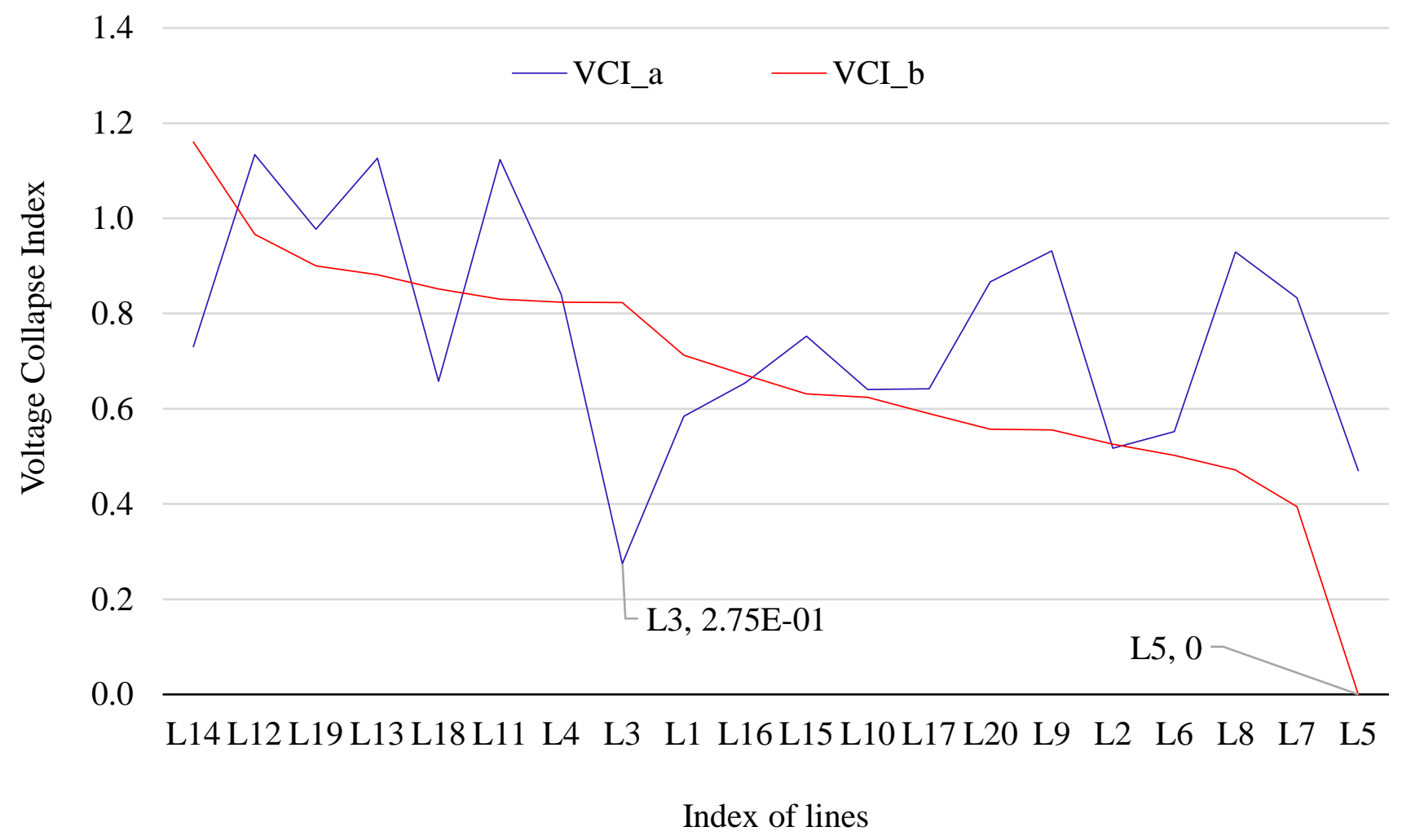

Figure 2.6 $V C I_{a}$ and $V C I_{b}$ for the 20 lines of the 14-bus system at VC.

Similarly, the Jacobian of the line-wise NR method yields VCI values at both ends of lines for the IEEE 118-bus system when the bus loads are scaled by 4.23 times as shown in Figure 2.7. It indicates VC at line 108 between busses 49 and 69 where $V C I_{a}$ and $V C I_{b}$ values are 2.35E-03 and 2.30E-02 respectively. 


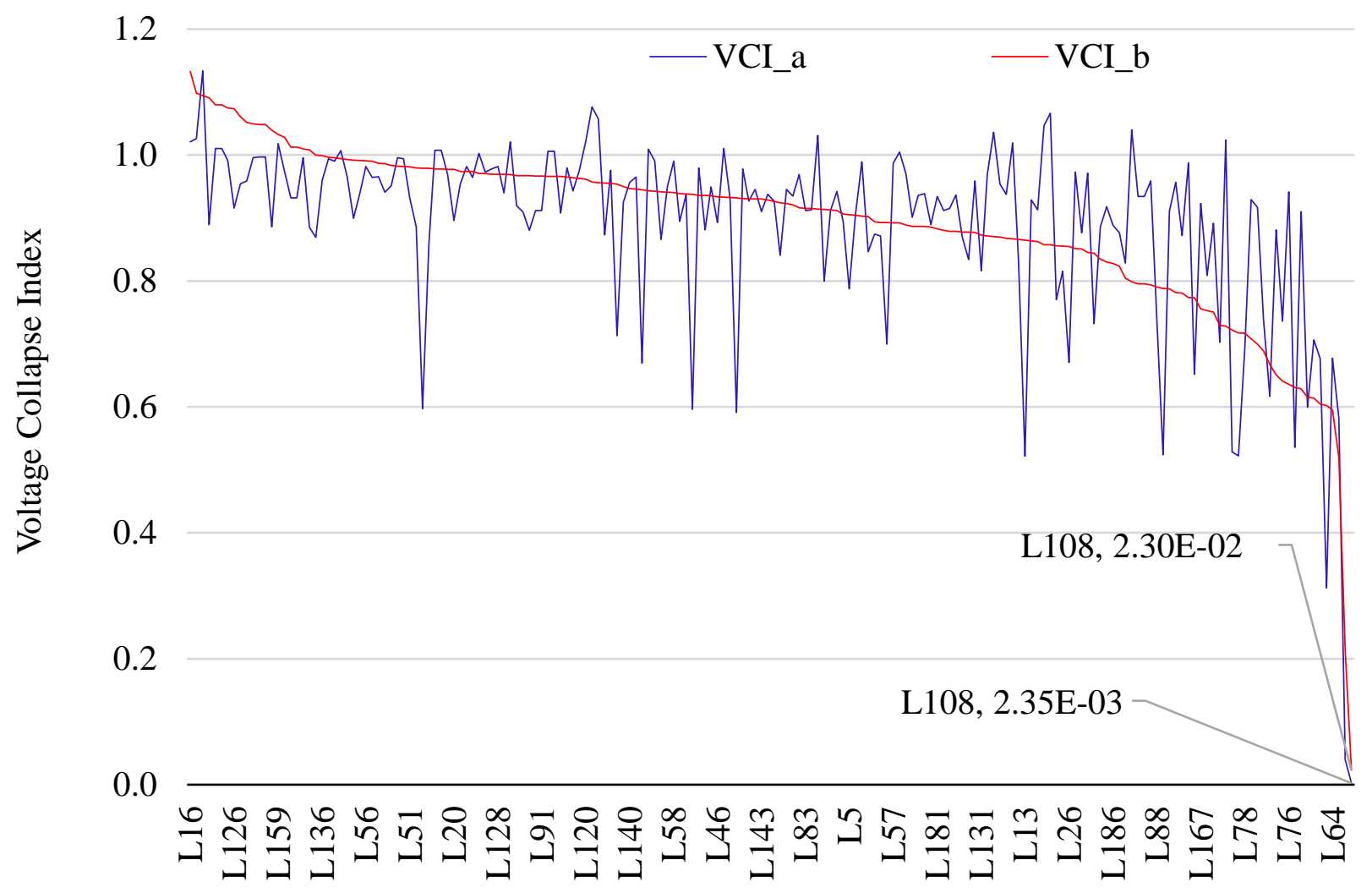

Index of lines

Figure $2.7 V C I_{a}$ and $V C I_{b}$ for the 186 lines of 118-bus system at VC.

For the 582-bus real power system, Figure 2.8 shows the values of $V C I$ at both ends of lines where the line 198 between busses 415 and 109 will collapse when the bus loads are scaled by 2.253 times, where $V C I_{a}$ and $V C I_{b}$ values are 6.37E-03 and 2.50E-01 respectively. Again, this determination is directly made by reviewing the Jacobian of the line-wise NR method. 


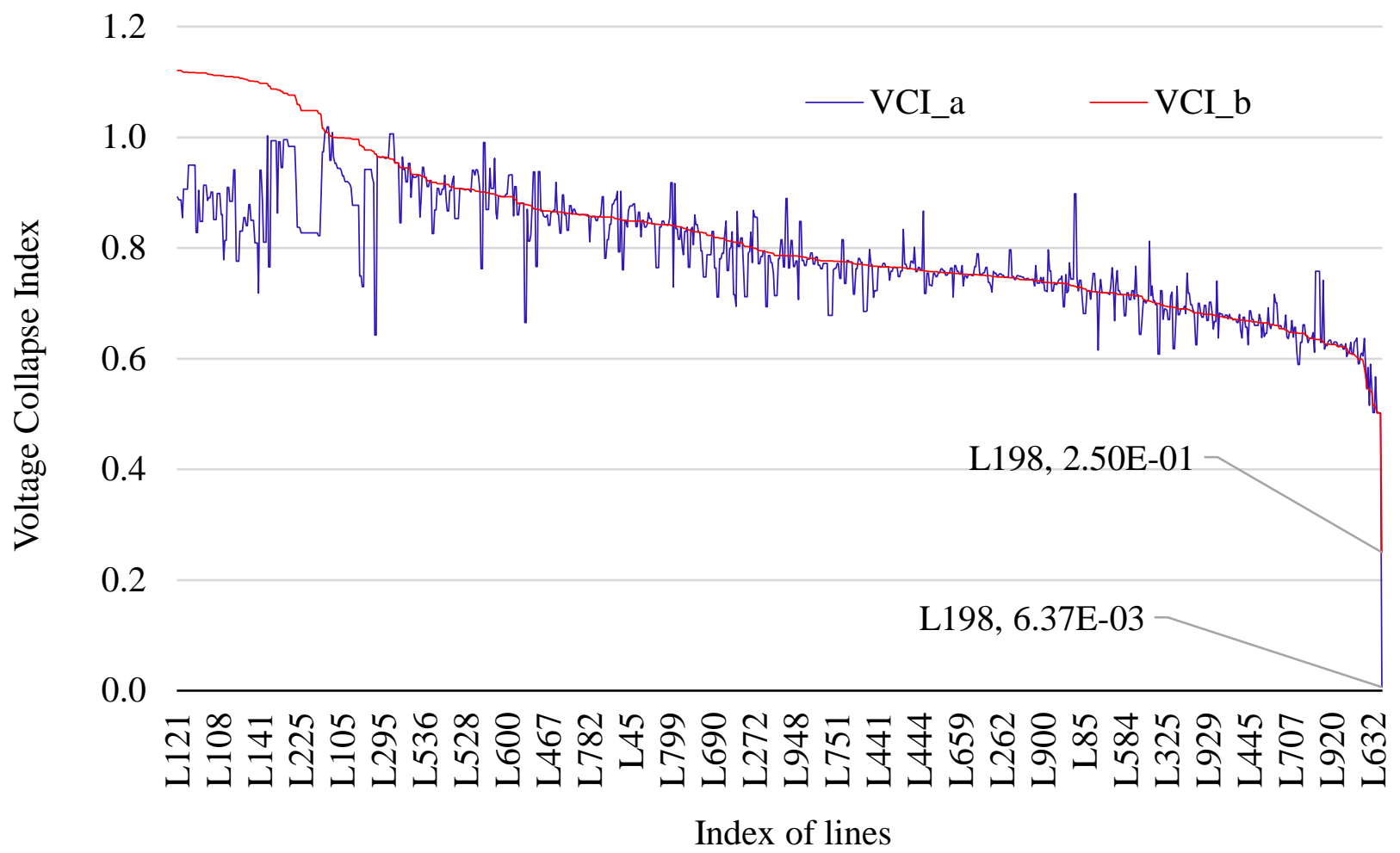

Figure $2.8 V C I_{a}$ and $V C I_{b}$ for the 965 lines of 582-bus system at VC.

Table 2.5 Value of Worst VCI and Computation Times for All the VCIs

\begin{tabular}{|c|c|c|c|c|c|c|}
\hline & \multicolumn{3}{|c|}{$\begin{array}{c}\text { Worst VCI } \\
\text { (minimum VCI at both sides) }\end{array}$} & \multicolumn{2}{|c|}{$\begin{array}{l}\text { Computation time } \\
\text { (milliseconds) }\end{array}$} \\
\hline & & $\begin{array}{l}\text { Bus-wise NR } \\
\text { method }\end{array}$ & $\begin{array}{l}\text { Line-wise NR } \\
\text { method }\end{array}$ & Difference & $\begin{array}{l}\text { Bus-wise NR } \\
\text { method }\end{array}$ & $\begin{array}{l}\text { Line-wise NR } \\
\text { method }\end{array}$ \\
\hline \multirow{2}{*}{$\begin{array}{l}14- \\
\text { bus }\end{array}$} & $\mathrm{VCI}_{\mathrm{a}}$ & 0.277 & 0.274 & 0.003 & \multirow{2}{*}{13.0} & \multirow{2}{*}{4.0} \\
\hline & $\mathrm{VCI}_{\mathrm{b}}$ & 0.0 & 0.0 & 0.0 & & \\
\hline \multirow{2}{*}{$\begin{array}{l}118- \\
\text { bus }\end{array}$} & $\mathrm{VCI}_{\mathrm{a}}$ & 0.00195 & 0.00235 & 0.0004 & \multirow{2}{*}{39.0} & \multirow{2}{*}{15.0} \\
\hline & $\mathrm{VCI}_{\mathrm{b}}$ & 0.029 & 0.023 & 0.006 & & \\
\hline \multirow{2}{*}{$\begin{array}{r}582- \\
\text { bus }\end{array}$} & $\mathrm{VCI}_{\mathrm{a}}$ & 0.006 & 0.0064 & 0.0004 & \multirow{2}{*}{421.0} & \multirow{2}{*}{152.0} \\
\hline & $\mathrm{VCI}_{\mathrm{b}}$ & 0.2512 & 0.2499 & 0.0013 & & \\
\hline
\end{tabular}


From the foregoing description, it is evident that $\mathrm{VC}$ indices $V C I_{a}$ and $V C I_{b}$ are directly available in the Jacobian of the line-wise NR method that helps to identify the set of critical lines that connect busses that are the most susceptible to VC without additional computation.

Table 2.5 provides the worst VCI values and times to compute hem for both the bus-wise NR method and the line-wise NR method. For all tested systems, first, the difference between the computed worst values of VCI for bus-wise NR versus line-wise NR methods is very low. It demonstrates the accuracy of the proposed method. Second, comparing the computational time to tally VCIs shows that the line-wise NR method is computationally equal or up to twice as fast as the bus-wise NR method. This data considers time to compute the voltage solution.

\subsection{Voltage Stability Contingency Analysis}

This section examines voltage stability contingency analysis. VC indices based upon the proposed linewise NR method are used to quantify the proximity to VC and rank several contingency states. Table 2.6 compares the traditional bus-wise NR method and the proposed line-wise NR method, measuring the total computation time required to solve power balance equations for each single line outage for 14-, 118-, and 300-bus IEEE systems and a 2383-bus Polish power system. The results show the superiority of the proposed line-wise NR method, which is computationally up to twice as fast as the bus-wise NR method. It is noted that the bus-wise NR method and the line-wise NR method produce VCI values very close to each other, as reported in Section 2.5, Table 2.6. Hence, the two methods rank the same set of contingencies as the most severe.

Table 2.6 Computation Times for All Single Line Outage

\begin{tabular}{c|c|c|c|c}
\hline \multirow{2}{*}{ System } & $\begin{array}{c}\text { Number of } \\
\text { contingency cases } \\
\text { (number of lines) }\end{array}$ & $\begin{array}{c}\text { Traditional } \\
\text { bus-wise NR } \\
\text { method } \\
\text { (seconds) }\end{array}$ & $\begin{array}{c}\text { Proposed } \\
\text { Line-wise NR } \\
\text { method } \\
\text { (seconds) }\end{array}$ & $\begin{array}{c}\text { Computation time } \\
\text { of line-wise method } \\
\text { as percentage of } \\
\text { bus-wise method }\end{array}$ \\
\hline 14-bus & 20 & 0.26 & 0.080 & $30.77 \%$ \\
\hline 57-bus & 80 & 2.24 & 0.988 & $44.11 \%$ \\
\hline 118-bus & 186 & 7.254 & 4.185 & $57.69 \%$ \\
\hline 300-bus & 411 & 49.32 & 22.194 & $45.00 \%$ \\
\hline
\end{tabular}


As an example, single line outage contingency analysis is performed on a 2383-bus Polish power systems. The results of the twenty most severe line outages are summarized in Table 2.7.

Table 2.7 Twenty Most Severe Single Outage Contingency Cases for Polish 2383-bus System

\begin{tabular}{|c|c|c|c|c|}
\hline \multirow{2}{*}{$\begin{array}{c}\text { Contingency } \\
\text { case/outage branches } \\
\text { (rank) }\end{array}$} & \multirow{2}{*}{ Worst VCI } & \multirow{2}{*}{$\begin{array}{l}\text { Most critical line } \\
\text { (connects buses } \\
\text { most susceptible to } \\
\text { VC) }\end{array}$} & \multicolumn{2}{|c|}{$\begin{array}{l}\text { Execution time } \\
\text { (seconds) }\end{array}$} \\
\hline & & & $\begin{array}{c}\text { Bus-wise NR } \\
\text { method }\end{array}$ & $\begin{array}{c}\text { Line-wise NR } \\
\text { method }\end{array}$ \\
\hline $169(1)$ & 0.1341 & L152-66 & 1.60 & 1.02 \\
\hline $2058(2)$ & 0.2081 & L1987-1981 & 1.57 & 0.90 \\
\hline $469(3)$ & 0.2113 & L437-188 & 1.57 & 1.02 \\
\hline $2761(4)$ & 0.2837 & L2209-2351 & 1.54 & 1.00 \\
\hline $2144(5)$ & 0.2906 & L2096-1912 & 1.54 & 1.01 \\
\hline $405(6)$ & 0.2927 & L434-188 & 1.57 & 1.03 \\
\hline $467(7)$ & 0.2981 & L431-229 & 1.47 & 1.04 \\
\hline $404(8)$ & 0.3261 & L431-229 & 1.57 & 1.04 \\
\hline $2030(9)$ & 0.3323 & L1822-1758 & 1.57 & 1.01 \\
\hline 777 (10) & 0.3328 & L1999-1744 & 1.55 & 0.99 \\
\hline $966(11)$ & 0.3350 & L630-601 & 1.57 & 1.02 \\
\hline $5(12)$ & 0.3395 & L431-229 & 1.57 & 1.02 \\
\hline $2767(13)$ & 0.3460 & L2310-2289 & 1.54 & 1.01 \\
\hline $2881(14)$ & 0.3487 & L2137-2348 & 1.57 & 1.03 \\
\hline $501(15)$ & 0.3503 & L466-230 & 1.57 & 1.03 \\
\hline $296(16)$ & 0.3552 & L1906-145 & 1.57 & 1.01 \\
\hline $513(17)$ & 0.3554 & L352-253 & 1.57 & 1.07 \\
\hline $292(18)$ & 0.3583 & L129-148 & 1.60 & 1.03 \\
\hline 2396 (19) & 0.3587 & L1992-1663 & 1.57 & 0.99 \\
\hline $2401(20)$ & 0.3658 & L1945-1850 & 1.57 & 1.00 \\
\hline
\end{tabular}




\subsection{Summary}

Bus-wise power balance equations and its solution using NR technique and its variants are numerically proven as powerful PF analysis tools, possessing high degrees of accuracy and speed. However, the buswise NR method is computationally intensive and does not readily lend itself to identifying the set of critical lines that connect the busses that are susceptible to VC.

In this chapter, a new line-wise set of power balance equations and its solution using NR technique is proposed. The formulation uses square of voltage magnitude instead of using voltage magnitudes alone.

The use of square of voltage magnitude leads to linear terms for real and reactive powers, considering constant impedance type elements in certain portion of power balance equations. Coupled with this fact and the use of real and reactive line-wise power flows as variables, the set of line-wise equations and its solution using NR technique is faster for all tested systems, up to two times, compared with bus-wise NR methods. Numerical analysis of the Jacobians of bus-wise and line-wise NR methods shows that the number of calculations to be completed by line-wise NR method is much less, resulting in the higher speed of the linewise NR method.

The proposed line-wise NR method is tested and compared for performance with the bus-wise NR method on 6-, 14-, 57- and 118-bus IEEE systems, a 582-bus real power system, a 2383-bus Polish power system, and a 9241-bus PEGASE system. The line-wise NR method demonstrates a stable numerical performance, monotonic convergence and solution accuracy. It scales well, as shown, for larger systems. The active and reactive line power flows result directly from the solution, with no further need for computation. The need to construct the system admittance matrix is eliminated. Development of the system Jacobian is straightforward and requires no knowledge of the system topology.

Another significant benefit of the proposed line-wise NR method is the ability to directly identify the set of critical lines that connect busses that are the most susceptible to VC. VCI terms appear directly in the Jacobian of the line-wise NR method. The method is both fast for PF analysis and for use as an online tool for voltage stability assessment. Summarizing, the benefits of the proposed line-wise NR method are:

(1) It is superior to the bus-wise power method as it solves faster up to 2 times.

(2) It demonstrates a stable numerical performance, monotonic convergence, solution accuracy and scales well for larger systems.

(3) It provides active and reactive line power flows with no further need for computation.

(4) It doesn't need the system admittance matrix. 
(5) Its system Jacobian construction is straightforward and requires no knowledge of the system topology.

(6) It provides VCI values without additional computation.

(7) It is very effective and time saving for voltage stability assessment analysis and ranking contingency scenarios according to their proximity to VC. 


\section{Chapter Three}

\section{Chapter 3: Rectangular Line-Wise PoWer Flow}

In the preceding chapter, it is shown that polar-form of line-wise power flow is better in several respects such as reduced computational burden, faster execution speed, better ability to report on voltage stability indices, etc. This work is now extended to development of line-wise power flow methods for other forms.

Accordingly, in this chapter, a set of line-wise of equations in rectangular form for modeling a power system and its solution method using NR technique for power flow analysis are proposed. Study results on several IEEE benchmark systems, as well as several real systems, up to a 9000-bus system, show that the proposed method is accurate, provides monotonic convergence, scales well for large systems and is consistently faster, up to thrice the speed of rectangular bus-wise NR method, while using sparse matrices. In the previous chapter, a novel line-wise PF is reported. The formulation is derived in the polar form which is based on the square of bus voltage magnitudes. In this chapter, a rectangular line-wise PF algorithm is proposed. The formulation comprising a set of rectangular line-wise power balance equations is presented. The set of equations is formulated in terms of the real and imaginary terms of bus voltages. It is solved using the NR technique. The solution directly yields bus-wise voltage solution and line-wise real and reactive power flows. The formulation easily scales for larger systems, demonstrates numerical stability, lends itself to sparse matrix technique, provides monotonic convergence, and consistently converges at a faster speed up to thrice the speed in comparison to the bus-wise NR method for PF analysis.

\subsection{Proposed line-wise PF system of equations}

In this section, the proposed rectangular formulation of the set of line-wise power balance equations is presented. Considering only the series impedance element of the pi-model of a transmission line or a transformer between buses $a$ and $b$, the circuit model is shown in Figure 3.1. 


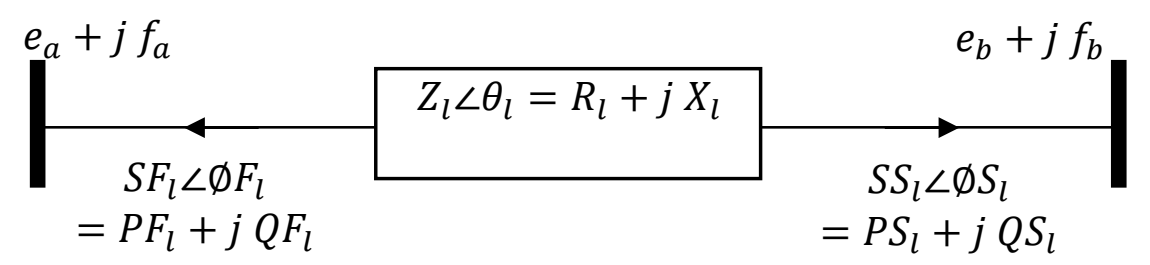

Figure 3.1 Series impedance element of the pi-model of the $l^{\text {th }}$ transmission branch connecting busses $a$ and $b$.

Based on [134, 137], using the voltage difference between busses $a$ and $b$, power flow in the first end can be expressed as:

$$
\left(e_{a}+j f_{a}\right)-\left(e_{b}+j f_{b}\right)=\left(\frac{S S_{l} \angle \emptyset S_{l}}{\left(e_{b}+j f_{b}\right)}\right)^{*} \cdot Z_{l} \angle \theta_{l}
$$

Multiplying both sides of (3.1) by the conjugate of $\left(e_{b}+j f_{b}\right)$ :

$$
\left(e_{a}+j f_{a}\right) \cdot\left(e_{b}-j f_{b}\right)-\left(e_{b}{ }^{2}+f_{b}^{2}\right)=\left(S S_{l} \angle \emptyset S_{l}\right)^{*} \cdot Z_{l} \angle \theta_{l}
$$

Separating the real and imaginary parts in (3.2)

$$
\begin{gathered}
\left(e_{b}^{2}+f_{b}^{2}\right)-\left(e_{a} \cdot e_{\mathrm{b}}+f_{a} \cdot f_{\mathrm{b}}\right)+P S_{l} \cdot R_{l}+Q S_{l} \cdot X_{l}=0 \\
\left(f_{a} \cdot e_{\mathrm{b}}-e_{a} \cdot f_{\mathrm{b}}\right)-P S_{l} \cdot X_{l}+Q S_{l} \cdot R_{l}=0
\end{gathered}
$$

Similarly, for the second end:

$$
\left(e_{b}+j f_{b}\right)-\left(e_{a}+j f_{a}\right)=\left(\frac{S F_{l} \angle \emptyset F_{l}}{\left(e_{a}+j f_{a}\right)}\right)^{*} \cdot Z_{l} \angle \theta_{l}
$$

Multiplying both sides of (3.5) by the conjugate of $\left(e_{a}+j f_{a}\right)$ :

$$
\left(e_{b}+j f_{b}\right) \cdot\left(e_{a}-j f_{a}\right)-\left(e_{a}{ }^{2}+f_{a}^{2}\right)=\left(S F_{l} \angle \varnothing F_{l}\right)^{*} \cdot Z_{l} \angle \theta_{l}
$$


Separating the real and imaginary parts in (3.6)

$$
\begin{gathered}
\left(e_{a}{ }^{2}+f_{a}{ }^{2}\right)-\left(e_{a} \cdot e_{\mathrm{b}}+f_{a} \cdot f_{\mathrm{b}}\right)+P F_{l} \cdot R_{l}+Q F_{l} \cdot X_{l}=0 \\
\left(f_{b} \cdot e_{\mathrm{a}}-e_{b} \cdot f_{\mathrm{a}}\right)-P F_{l} \cdot X_{l}+Q F_{l} \cdot R_{l}=0
\end{gathered}
$$

Defining a matrix $[M]$ with a dimension of $N B \times 2 \cdot N T$, it is constructed as below by using the concept of bus incidence matrix:

$$
\begin{array}{ll}
{[M]_{a, l}=1} & \text { if bus } a \text { is the first bus for line } l \\
{[M]_{b, N T+l}=1} & \text { if bus } b \text { is the second bus for line } l \\
{[M]_{a, l}=0} & \text { otherwise }
\end{array}
$$

Collating (3.3), (3.4), (3.7) and (3.8) to model the entire transmission system, the following equations can be written as below. Consider a power system with $N G$ generator buses and $N L B$ load buses such that the total number of buses $N B=N G+N L B$ and $N T$ is the total number of transmission branches, the set of line-wise equations are defined below.

Using (3.3): $F 1_{l}=$

$$
\left(e_{b}^{2}+f_{b}^{2}\right)-\left(e_{a} \cdot e_{\mathrm{b}}+f_{a} \cdot f_{\mathrm{b}}\right)+P S_{l} \cdot R_{l}+Q S_{l} \cdot X_{l}=0 \quad \forall l=1 \text { to } N T
$$

Using (3.7): $F 2_{l}=$

$$
\left(e_{a}{ }^{2}+f_{a}^{2}\right)-\left(e_{a} \cdot e_{\mathrm{b}}+f_{a} \cdot f_{\mathrm{b}}\right)+P F_{l} \cdot R_{l}+Q F_{l} \cdot X_{l}=0 \quad \forall l=1 \text { to } N T
$$

Using (3.4): $F 3_{l}=$

$$
\left(f_{a} \cdot e_{\mathrm{b}}-e_{a} \cdot f_{\mathrm{b}}\right)-P S_{l} \cdot X_{l}+Q S_{l} \cdot R_{l}=0 \quad \forall l=1 \text { to } N T
$$

Using (3.8): $F 4_{l}=$

$$
\left(f_{b} \cdot e_{\mathrm{a}}-e_{b} \cdot f_{\mathrm{a}}\right)-P F_{l} \cdot X_{l}+Q F_{l} \cdot R_{l}=0 \quad \forall l=1 \text { to } N T
$$

Further, the bus-wise power balance equations, can be written in a matrix form as below:

$$
\begin{array}{r}
F 5=[M]\left[\begin{array}{l}
P F \\
P S
\end{array}\right]-\left(e^{2}+f^{2}\right) \cdot G S=P D-P G \quad \text { for all busses except the slack bus } \\
F 6=[M]\left[\begin{array}{l}
Q F \\
Q S
\end{array}\right]+\left(e^{2}+f^{2}\right) \cdot B S=Q D-Q G \quad \text { for all load busses }
\end{array}
$$

In addition, the square of voltage magnitudes of generation buses can be written as in (15):

$$
F 7=\left(e^{2}+f^{2}\right)=V_{S H}{ }^{2} \quad \text { for all non }- \text { slack generator busses }
$$

where the following are noted. 
(1) Shunt elements of pi-model of transmission lines and transformer are included into $G$ and $B$, vectors of bus-wise conductance and susceptance elements, in (3.13) and (3.14).

(2) Constant power loads are modeled into $P D$ and $Q D$ of (3.13) and (3.14).

(3) Constant impedance loads, if any, are included into $G S$ and $B S$, vectors of bus-wise conductance and susceptance elements, in (3.13) and (3.14).

The set of equations (3.9) to (3.15) models a power system. The total number of variables in this set of equations is $4 \cdot N T+2 \cdot N B-2$ and it equals the number of equations.

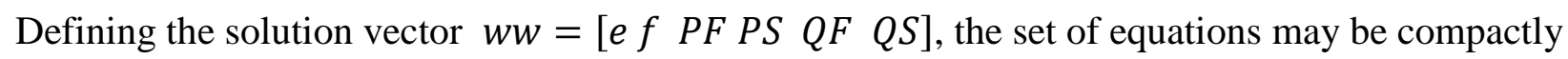
presented as below:

$$
\begin{gathered}
{\left[\begin{array}{l}
F 1(w w) \\
F 2(w w) \\
F 3(w w) \\
F 4(w w) \\
F 5(w w) \\
F 6(w w) \\
F 7(w w)
\end{array}\right]=\left[\begin{array}{c}
0 \\
0 \\
0 \\
0 \\
{[P D-P G]} \\
{[Q D-Q G]} \\
V_{S H}{ }^{2}
\end{array}\right]} \\
F 8(w w)=S R
\end{gathered}
$$$$
\text { or }
$$

This set of nonlinear equations can be easily solved using Newton-Raphson technique. The following section provides a solution method.

\subsection{Proposed algorithm}

The system of equations (3.16) can be solved to determine the voltage solution using NR technique. This section describes the proposed algorithm to solve a set of equations that describe a power system using a multivariate NR technique. The method readily scales for larger systems and demonstrates a stable performance. Figure 3.2 shows the flowchart of the proposed technique. 


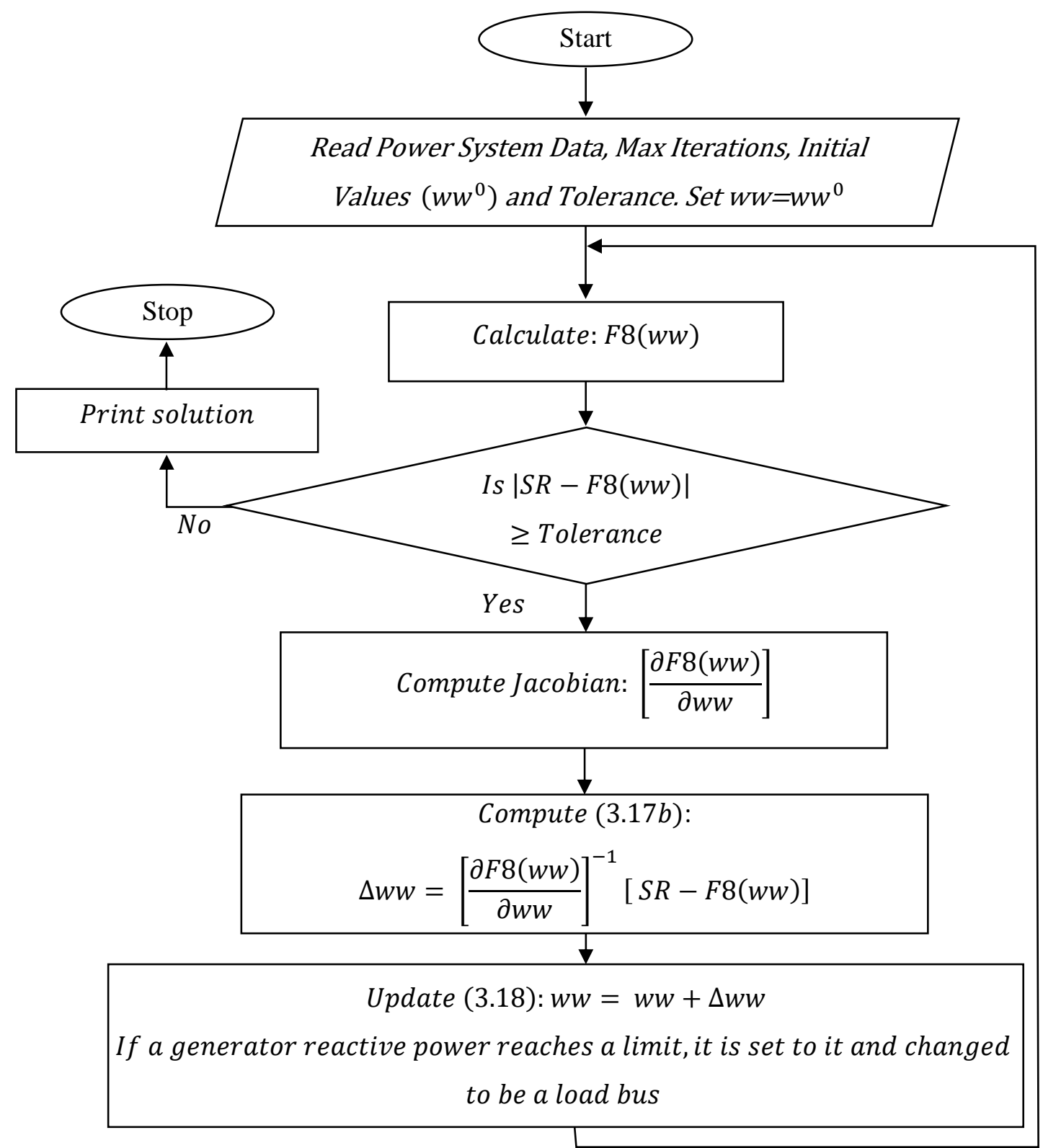

Figure 3.2 Flowchart of proposed algorithm.

It should be noted that the solution steps are similar to the standard bus-wise NR method for PF analysis, except that the set of equations to be solved is the set of rectangular line-wise power balance equations. In addition, there is no need to construct the bus admittance matrix for the line-wise NR method, which is required for the bus-wise NR method. 
Using Taylor's series expansion and limiting to the first order derivative while considering a solution $w w,(3.16)$ can be expanded as below:

$$
\begin{gathered}
{\left[\begin{array}{l}
F 1(w w) \\
F 2(w w) \\
F 3(w w) \\
F 4(w w) \\
F 5(w w) \\
F 6(w w) \\
F 7(w w)
\end{array}\right]+\left[\begin{array}{l}
\partial F 1(w w) / \partial w w \\
\partial F 2(w w) / \partial w w \\
\partial F 3(w w) / \partial w w \\
\partial F 4(w w) / \partial w w \\
\partial F 5(w w) / \partial w w \\
\partial F 6(w w) / \partial w w \\
\partial F 7(w w) / \partial w w
\end{array}\right] \cdot[\Delta w w]=\left[\begin{array}{c}
0 \\
0 \\
0 \\
0 \\
{[P D-P G]} \\
{[Q D-Q G]} \\
V_{S H}{ }^{2}
\end{array}\right]} \\
F R(w w)+\left[\frac{\partial F R(w w)}{\partial w w}\right] \cdot \Delta w w=S R
\end{gathered}
$$

where $\left[\frac{\partial F R(w w)}{\partial w w}\right]$ is the Jacobian of the set of equations (3.9) - (3.15). In case $w w$ does not satisfy the set of equations (3.16), then using (3.17a), an update for $w w$ can be computed as below:

$$
\begin{aligned}
& \Delta w w=\left[\frac{\partial F R(w w)}{\partial w w}\right]^{-1} \cdot[S R-F R(w w)] \quad \text { or } \\
& \Delta w w=\left[\frac{\partial F R(w w)}{\partial w w}\right]^{-1} \cdot[\Delta F R(w w)]
\end{aligned}
$$

where $\Delta F R=[\Delta F 1 \Delta F 2 \Delta F 3 \Delta F 4 \Delta F 5 \Delta F 6 \Delta F 7]^{T}$ and $\Delta w w=[\Delta e \Delta f \Delta P F \Delta P S \Delta Q F \Delta Q S]$. On computing $\Delta w w$, the solution vector can be updated as:

$$
w w=w w+\Delta w
$$

By repeating computation of (3.17b) and (3.18) until the absolute value of all terms within $[S R-F 8(w w)]$ reduce to a zero or below an acceptably small value, the solution vector $w w$ for the set of equations (3.16) is obtained. 
It must be noted that the following are known:

(1) real and imaginary terms of voltage at the slack bus,

(2) real power injections (generation minus load) and bus voltage magnitudes at all generation busses, and,

(3) real and reactive power injections (negative of real and reactive power loads) at load busses.

The unknowns then are:

(1) real and reactive power injections at the slack bus,

(2) real and imaginary terms of voltage as well as the reactive power injections at generator busses, and

(3) real and imaginary terms of voltage at load busses.

Knowns and unknowns are summarized in a tabular form below:

\begin{tabular}{c|c|c}
\hline & Knowns & Unknowns \\
\hline \multirow{2}{*}{ Slack Bus } & $e$ & $P T=P G-P D$ \\
& $f$ & $Q T=Q G-Q D$ \\
\hline Generator Bus & $P T=P G-P D$ & $Q T=Q G-Q D$ \\
& $V_{S H}$ & $e$ and $f$ \\
\hline Load Bus & $P T=-P D$ & $e$ \\
& $Q T=-Q D$ & $f$ \\
\hline
\end{tabular}

Hence, given that real power injection is unknown for the slack bus, (3.13), i.e., $F 5(w w)=P D-P G$, must be solved for all busses except the slack bus.

Similarly, given that reactive power injections are unknown for the all generator busses (including the slack bus), (3.14), i.e., $F 6(w w)=Q D-Q G$, must be solved only for load busses.

Equations (3.9) to (3.12) for $F 1(w w), F 2(w w), F 3(w w)$, and $F 4(w w)$ have $N T$ equations each. Hence, the number of equations to be solved and the number of unknowns are as below: 


\begin{tabular}{c|c}
\hline Equations & Number \\
\hline$F 1(w w)$ & $N T$ \\
\hline$F 2(w w)$ & $N T$ \\
\hline$F 3(w w)$ & $N T$ \\
\hline$F 4(w w)$ & $N T$ \\
\hline$F 5(w w)$ & $N B-1$ \\
\hline$F 6(w w)$ & $N B-N G$ \\
\hline$F 7(w w)$ & $N G-1$ \\
\hline
\end{tabular}

\begin{tabular}{c|c}
\hline Unknowns & Number \\
\hline$P F$ & $N T$ \\
\hline$P S$ & $N T$ \\
\hline$Q F$ & $N T$ \\
\hline$Q S$ & $N T$ \\
\hline$e$ & $N B-1$ \\
\hline$f$ & $N$ \\
\hline
\end{tabular}

The total number of equations is $(4 \cdot N T+2 \cdot N B-2)$. The total number of unknowns is $(4 \cdot N T+2 \cdot N B-2)$.

The result of the proposed algorithm will be:

(1) real and imaginary terms of voltage at load busses,

(2) voltage phase angles at all busses except the slack bus, and,

(3) real and reactive powers on both ends for all branches (lines and transformers).

Details of the Jacobian of the set of equations (3.9) to (3.15), $\left[\frac{\partial F 8(w w)}{\partial w w}\right]$, are provided in the next section.

\subsection{Development of the Jacobian Matrix}

This section presents the mathematical development of the Jacobian matrix, which is the partial differentiation for the set of equations (3.9) - (3.15) with respect to the six unknown vectors [ e f PF PS QF QS ]. The complete system Jacobian is presented below considering (3.17b). The size of the submatrices of the system Jacobian is also documented below. 


\begin{tabular}{|c|c|c|c|c|c|}
\hline$\left\lceil\frac{\partial \mathrm{F} 1}{\partial \mathrm{e}}\right]$ & {$\left[\frac{\partial \mathrm{F} 1}{\partial \mathrm{f}}\right]$} & {$\left[\frac{\partial \mathrm{F} 1}{\partial \mathrm{PF}}\right]$} & $\left\lceil\frac{\partial F 1}{\partial P S}\right]$ & {$\left[\frac{\partial \mathrm{F} 1}{\partial \mathrm{QF}}\right]$} & {$\left[\frac{\partial F 1}{\partial Q S}\right]$} \\
\hline$N T \times N L B$ & $N T \times(N B-1)$ & $N T \times N T$ & $N T \times N T$ & $N T \times N T$ & $N T \times N T$ \\
\hline$[\partial \mathrm{F} 2]$ & {$[\partial F 2]$} & {$[\partial F 2]$} & {$[\partial \mathrm{F} 2]$} & {$[\partial F 2]$} & {$[\partial \mathrm{F} 2]$} \\
\hline$[\overline{\partial \mathrm{e}}]$ & {$[\overline{\partial f}]$} & $\lfloor\overline{\partial P F}\rfloor$ & $\lfloor\overline{\partial P S}\rfloor$ & $\lfloor\overline{\partial \mathrm{QF}}\rfloor$ & $\lfloor\overline{\partial Q S}\rfloor$ \\
\hline$N T \times N L B$ & $N T \times(N B-1)$ & $N T \times N T$ & $N T \times N T$ & $N T \times N T$ & $N T \times N T$ \\
\hline$\partial \mathrm{F} 3]$ & $\partial \mathrm{F} 3]$ & {$[\partial \mathrm{F} 3]$} & {$[\partial \mathrm{F} 3]$} & {$[\partial \mathrm{F} 3]$} & {$[\partial \mathrm{F} 3]$} \\
\hline$[\overline{\partial \mathrm{e}}]$ & {$[\overline{\partial \mathrm{f}}]$} & $\lfloor\overline{\partial \mathrm{PF}}\rfloor$ & $\lfloor\overline{\partial P S}\rfloor$ & $\lfloor\overline{\partial \mathrm{QF}}\rfloor$ & $\lfloor\overline{\partial Q S}\rfloor$ \\
\hline$N T \times N L B$ & $N T \times(N B-1)$ & $N T \times N T$ & $N T \times N T$ & $N T \times N T$ & $N T \times N T$ \\
\hline$[\partial \mathrm{F} 4]$ & $\partial \mathrm{F} 4]$ & {$[\partial \mathrm{F} 4]$} & {$[\partial \mathrm{F} 4]$} & {$[\underline{\partial \mathrm{F} 4}]$} & {$[\underline{\partial \mathrm{F} 4}]$} \\
\hline$[\overline{\partial \mathrm{e}}]$ & {$[\overline{\partial \mathrm{f}}]$} & $\lfloor\overline{\partial \mathrm{PF}}\rfloor$ & $\lfloor\overline{\partial P S}\rfloor$ & $\lfloor\overline{\partial \mathrm{QF}}\rfloor$ & $\lfloor\overline{\partial Q S}\rfloor$ \\
\hline$N T \times N L B$ & $N T \times(N B-1)$ & $N T \times N T$ & $N T \times N T$ & $N T \times N T$ & $N T \times N T$ \\
\hline$\underline{\partial \mathrm{F} 5}$ & $\underline{\partial F 5}$ & {$[\underline{\partial F} 5$} & $\underline{\partial \mathrm{F} 5}$ & {$\left[\frac{\partial F 5}{\square}\right]$} & $\lceil\underline{\partial F 5}]$ \\
\hline$[\overline{\partial \mathrm{e}}]$ & {$[\overline{\partial f}]$} & $\lfloor\overline{\partial \mathrm{PF}}]$ & $\lfloor\overline{\partial P S}\rfloor$ & $\lfloor\overline{\partial \mathrm{QF}}\rfloor$ & {$[\overline{\partial Q S}]$} \\
\hline$(N B-1)$ & $(N B-1) \times(N B$ & $(N B-1)$ & $(N B-1)$ & $(N B-1)$ & $(N B-1)$ \\
\hline$\times N L B$ & $-1)$ & $\times N T$ & $\times N T$ & $\times N T$ & $\times N T$ \\
\hline$\partial \mathrm{F} 6$ & $\underline{\partial F 6}$ & {$[\partial F 6$} & {$\left[\frac{\partial F 6}{0}\right]$} & {$\left[\frac{\partial \mathrm{F} 6}{]}\right]$} & $\underline{\partial \mathrm{F} 6}$ \\
\hline$[\overline{\partial \mathrm{e}}]$ & {$[\overline{\partial f}]$} & $\lfloor\overline{\partial \mathrm{PF}}\rfloor$ & $\lfloor\overline{\partial P S}\rfloor$ & $\lfloor\overline{\partial \mathrm{QF}}\rfloor$ & $\lfloor\overline{\partial Q S}\rfloor$ \\
\hline$N L B \times N L B$ & $N L B \times(N B-1)$ & $N L B \times N T$ & $N L B \times N T$ & $N L B \times N T$ & $N L B \times N T$ \\
\hline$[\partial \mathrm{F} 7]$ & {$[\partial F 7$} & {$[\partial F 7]$} & {$[\partial F 7]$} & {$[\underline{\partial \mathrm{F} 7}]$} & {$[\partial \mathrm{F} 7]$} \\
\hline$[\overline{\partial \mathrm{e}}]$ & {$[\overline{\partial f}]$} & $\lfloor\overline{\partial \mathrm{PF}}\rfloor$ & $\lfloor\overline{\partial P S}\rfloor$ & $\lfloor\overline{\partial \mathrm{QF}}]$ & $\lfloor\overline{\partial Q S}\rfloor$ \\
\hline$N G \times N L B$ & $N L B \times(N B-1)$ & $N L B \times N T$ & $N L B \times N T$ & $N L B \times N T$ & $N L B \times N T$ \\
\hline
\end{tabular}

It can be noted that out of 42 submatrices, there are 16 null submatrices. The following are null submatrices in the Jacobian:

$\left[\frac{\partial \mathrm{F} 1}{\partial \mathrm{PF}}\right],\left[\frac{\partial \mathrm{F} 1}{\partial \mathrm{QF}}\right],\left[\frac{\partial \mathrm{F} 2}{\partial \mathrm{PS}}\right],\left[\frac{\partial \mathrm{F} 2}{\partial \mathrm{QS}}\right],\left[\frac{\partial \mathrm{F} 3}{\partial \mathrm{PF}}\right],\left[\frac{\partial \mathrm{F} 3}{\partial \mathrm{QF}}\right],\left[\frac{\partial \mathrm{F} 4}{\partial \mathrm{PS}}\right],\left[\frac{\partial \mathrm{F} 4}{\partial \mathrm{QS}}\right],\left[\frac{\partial \mathrm{F} 5}{\partial \mathrm{QF}}\right],\left[\frac{\partial \mathrm{F} 5}{\partial \mathrm{QS}}\right],\left[\frac{\partial \mathrm{F} 6}{\partial \mathrm{PF}}\right],\left[\frac{\partial \mathrm{F} 6}{\partial \mathrm{PS}}\right],\left[\frac{\partial \mathrm{F} 7}{\partial \mathrm{PF}}\right],\left[\frac{\partial \mathrm{F} 7}{\partial \mathrm{QF}}\right]$, $\left[\frac{\partial \mathrm{F} 7}{\partial \mathrm{PS}}\right]$, and, $\left[\frac{\partial \mathrm{F} 7}{\partial \mathrm{QS}}\right]$. 
The non-zero elements of partial differentiation of (3.9) with respect to $e, f, \mathrm{PF}, \mathrm{PS}, \mathrm{QF}, \mathrm{QS}$ for $l \in$ $\{1,2, \ldots, N T\}$ are as follows:

$$
\begin{array}{ll}
\frac{\partial F 1}{\partial e_{a}}=-e_{b} & \forall\{a, b\} \in l \\
\frac{\partial F 1}{\partial e_{b}}=2 \cdot e B-e_{a} & \forall\{a, b\} \in l \\
\frac{\partial F 1}{\partial f_{a}}=-f_{b} & \forall\{a, b\} \in l \\
\frac{\partial F 1}{\partial f_{b}}=2 \cdot f_{b}-f_{a} & \forall\{a, b\} \in l \\
\frac{\partial F 1}{\partial P S}=R_{l} & \forall\{a, b\} \in l \\
\frac{\partial F 1}{\partial Q S}=X_{l} & \forall\{a, b\} \in l
\end{array}
$$

The non-zero elements of partial differentiation of (3.10) with respect to $e, f, \mathrm{PF}, \mathrm{PS}, \mathrm{QF}, \mathrm{QS}$ for $l \in$ $\{1,2, \ldots, N T\}$ are as follows:

$$
\begin{array}{ll}
\frac{\partial F 2}{\partial e_{a}}=2 \cdot e_{a}-e_{b} & \forall\{a, b\} \in l \\
\frac{\partial F 2}{\partial e_{b}}=-e_{a} & \forall\{a, b\} \in l \\
\frac{\partial F 2}{\partial f_{a}}=2 \cdot f_{a}-f_{b} & \forall\{a, b\} \in l \\
\frac{\partial F 2}{\partial f_{b}}=-f_{a} & \forall\{a, b\} \in l \\
\frac{\partial F 2}{\partial P F}=R_{l} & \forall\{a, b\} \in l \\
\frac{\partial F 2}{\partial Q F}=X_{l} & \forall\{a, b\} \in l
\end{array}
$$

The non-zero elements of partial differentiation of (3.11) with respect to $e, f, \mathrm{PF}, \mathrm{PS}, \mathrm{QF}, \mathrm{QS}$ for $\forall l \in$ $\{1,2, \ldots, N T\}$ are below:

$$
\frac{\partial F 3}{\partial e_{a}}=-f_{b} \quad \forall\{a, b\} \in l
$$




$$
\begin{array}{ll}
\frac{\partial F 3}{\partial e_{b}}=f_{a} & \forall\{a, b\} \in l \\
\frac{\partial F 3}{\partial f_{a}}=e_{b} & \forall\{a, b\} \in l \\
\frac{\partial F 3}{\partial f_{b}}=-e_{a} & \forall\{a, b\} \in l \\
\frac{\partial F 3}{\partial P S}=X_{l} & \forall\{a, b\} \in l \\
\frac{\partial F 3}{\partial Q S}=-R_{l} & \forall\{a, b\} \in l
\end{array}
$$

The non-zero elements of partial differentiation of (3.12) with respect to $e, f, \mathrm{PF}, \mathrm{PS}, \mathrm{QF}, \mathrm{QS}$ for $\forall l \in$ $\{1,2, \ldots, N T\}$ are below:

$$
\begin{array}{ll}
\frac{\partial F 4}{\partial e_{a}}=f_{b} & \forall\{a, b\} \in l \\
\frac{\partial F 4}{\partial e_{b}}=-f_{a} & \forall\{a, b\} \in l \\
\frac{\partial F 4}{\partial f_{a}}=-e_{b} & \forall\{a, b\} \in l \\
\frac{\partial F 4}{\partial f_{b}}=e_{a} & \forall\{a, b\} \in l \\
\frac{\partial F 4}{\partial P F}=-X_{l} & \forall\{a, b\} \in l \\
\frac{\partial F 4}{\partial Q F}=r_{l} & \forall\{a, b\} \in l
\end{array}
$$

The non-zero elements of partial differentiation of (3.13) with respect to $e, f, \mathrm{PF}, \mathrm{PS}, \mathrm{QF}, \mathrm{QS}$ for $\forall a \in$ $\{1,2, \ldots, N B\}$ are below:

$$
\begin{array}{ll}
\frac{\partial F 5}{\partial e}=-2 \cdot e \cdot G S_{a} & \forall a \in\{1,2, \ldots, N B\} \\
\frac{\partial F 5}{\partial f}=-2 \cdot f \cdot G S_{a} & \forall a \in\{1,2, \ldots, N B\}
\end{array}
$$


And for $l \in\{1,2, \ldots, N T\}$ as below:

$$
\begin{array}{ll}
\frac{\partial F 5}{\partial P F}=[M F]_{a, l} & \forall\{a, b\} \in l \\
\frac{\partial F 5}{\partial P S}=[M T]_{b, N T+l} & \forall\{a, b\} \in l
\end{array}
$$

The non-zero elements of partial differentiation of (3.14) with respect to $e, f, \mathrm{PF}, \mathrm{PS}, \mathrm{QF}, \mathrm{QS}$ for $\forall a \in$ $\{1,2, \ldots, N B\}$ are below:

$$
\begin{array}{ll}
\frac{\partial F 6}{\partial e}=2 \cdot e \cdot B S_{a} & \forall\{a, b\} \in l \\
\frac{\partial F 6}{\partial f}=2 \cdot f \cdot B S_{a} & \forall\{a, b\} \in l
\end{array}
$$

And for $l \in\{1,2, \ldots, N T\}$ as below:

$$
\begin{array}{ll}
\frac{\partial F 6}{\partial Q F}=[M F]_{a, l} & \forall\{a, b\} \in l \\
\frac{\partial F 6_{b}}{\partial Q S}=[M T]_{b, N T+l} & \forall\{a, b\} \in l
\end{array}
$$

The non-zero elements of partial differentiation of (3.15) with respect to $e, f, \mathrm{PF}, \mathrm{PS}, \mathrm{QF}, \mathrm{QS}$ for $\forall a \in$ $\{1,2, \ldots, N B\}$ are below:

$$
\begin{aligned}
& \frac{\partial F 7}{\partial e}=2 \cdot e \\
& \frac{\partial F 7}{\partial f}=2 \cdot f
\end{aligned}
$$

The Jacobian matrix of the proposed set of equations is a sparse matrix leading to a low computation burden. The overall dimension of the matrix is $(4 \cdot N T+2 \cdot N B-2) \times(4 \cdot N T+2 \cdot N B-2)$.

Figure 3.3 and Figure 3.4 show the sparsity patterns of Jacobian matrix for a 6-bus and 118-bus systems respectively. 


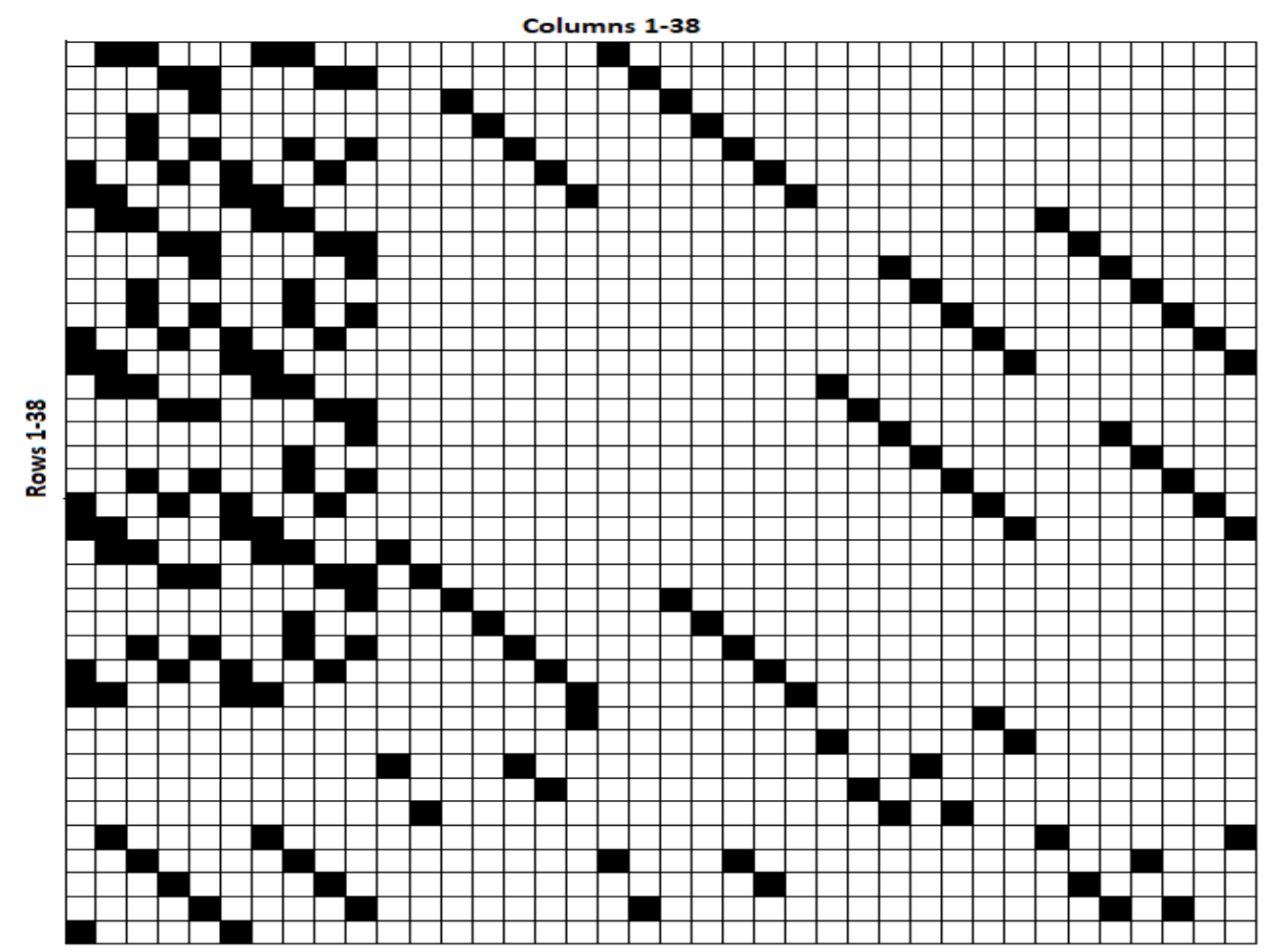

Figure 3.3 Plot of 6-bus System Jacobian matrix.

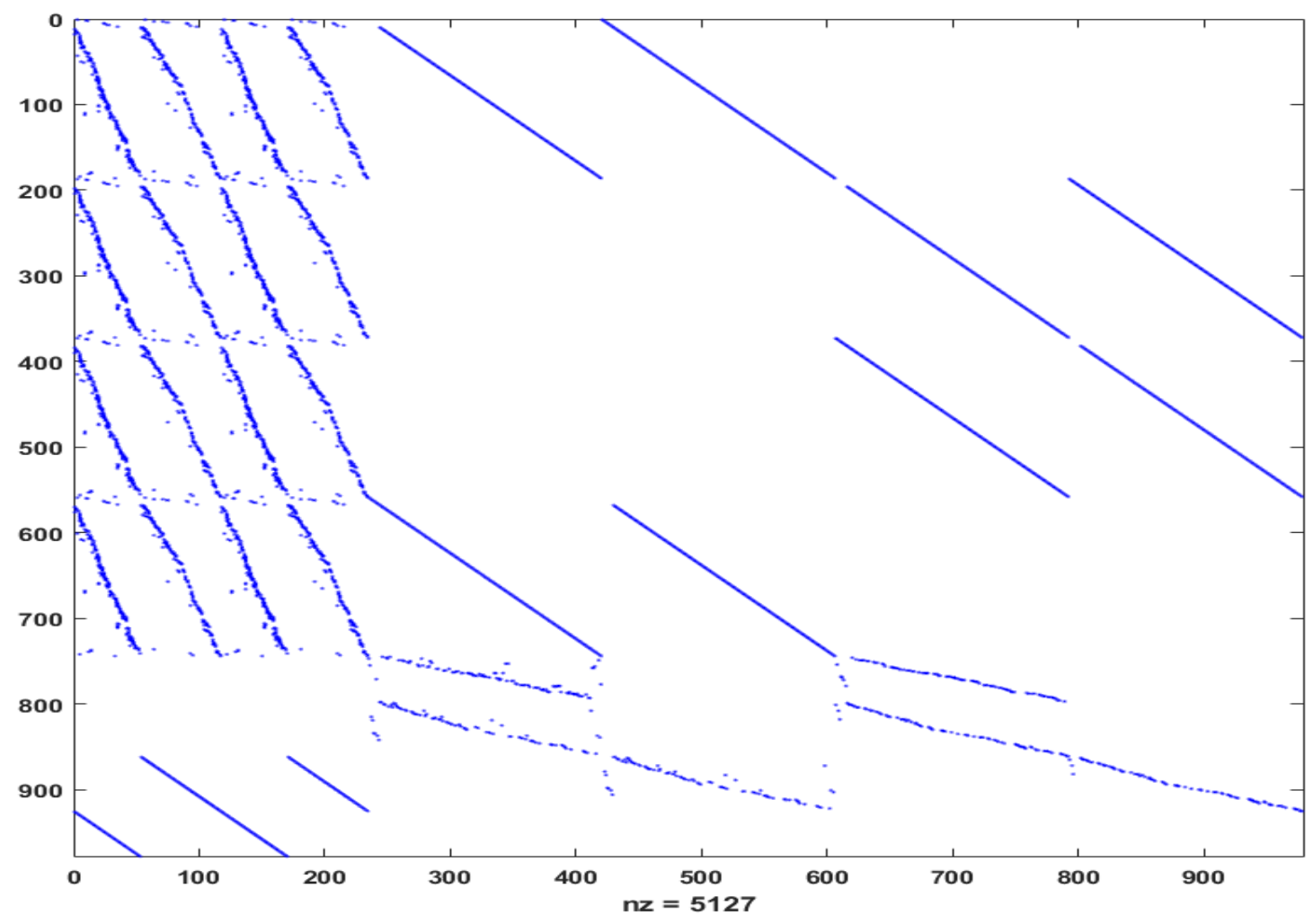

Figure 3.4 Sparse matrix of 118-bus System Jacobian matrix. 


\subsection{Results of System Studies}

In this section, test results of the proposed line-wise NR method are reported and compared with those from the bus-wise NR method. Both proposed rectangular line-wise and rectangular bus-wise NR method algorithms are coded in MATLAB (version R2016a). All tests are done on a 64-bit i7 Intel Core laptop (2.6 $\mathrm{GHz}, 16 \mathrm{~GB}$ of RAM) with Windows 10 operating system. Sparse matrix implementations are considered where possible.

Loads are modeled as constant MVA loads, and lines and transformers are modeled as pi-models considering their shunt admittance elements. Convergence tolerance is set to $10^{-5}$ per-unit.

To demonstrate the quality of solution from the proposed rectangular line-wise NR method, the difference in results between the traditional rectangular bus-wise NR method and the line-wise NR method for real and imaginary voltage terms are determined and quantified as Root Mean Square Error (RMSE) values. These RMSE values are reported in Figure 3.5 for the 6-, 14-, 57-, 118-bus IEEE systems, a 582bus real system, a 2383-bus Polish power system, and a 9241-bus PEGASE system. With very low RMSE values, the comparison shows that the proposed method has an accurate performance in terms of solution accuracy.

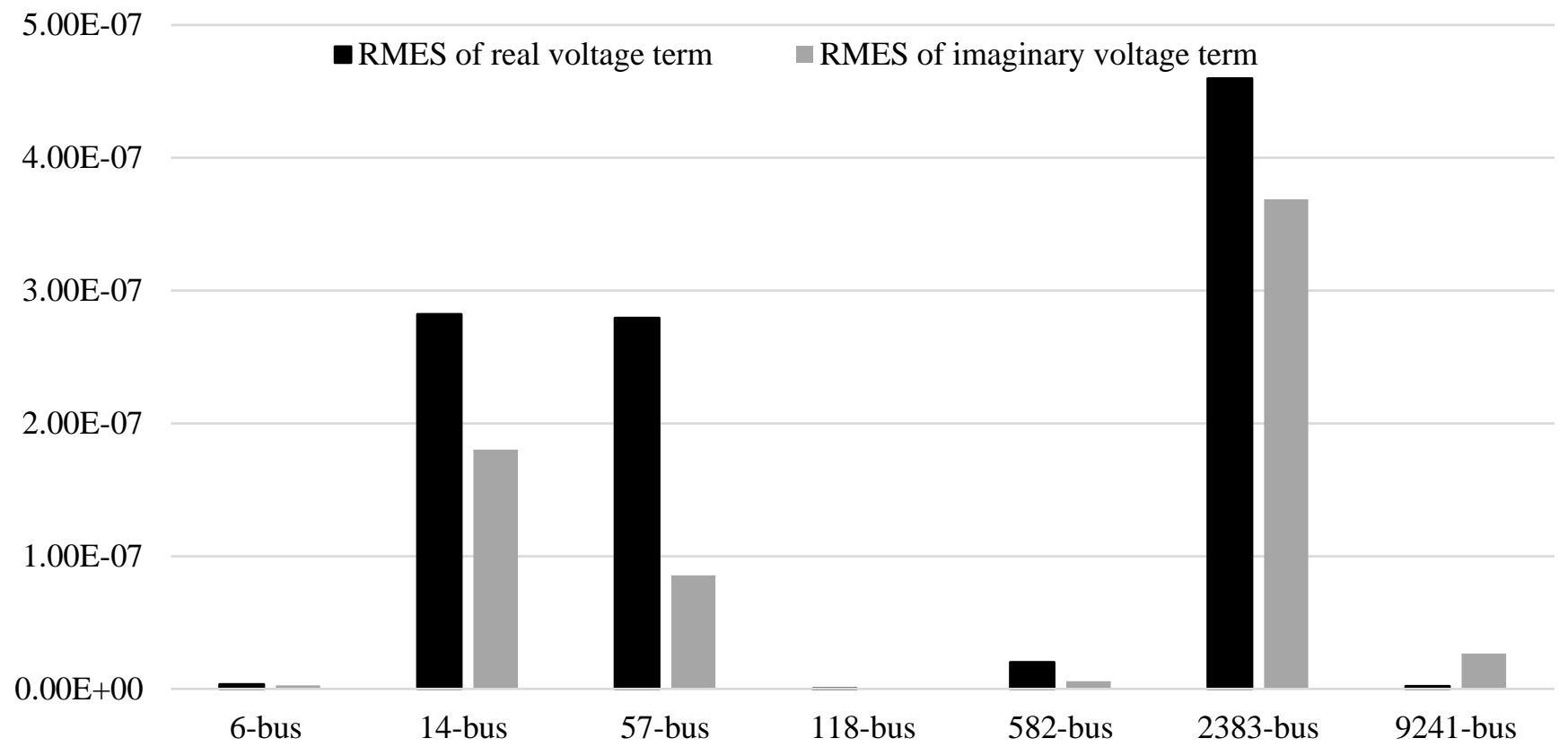

Figure 3.5 The Root Mean Square Error (RMSE) of bus real and imaginary voltage terms for difference in results between PF solutions of the traditional rectangular bus-wise and the proposed rectangular line-wise NR methods. 
The convergence characteristics of line-wise NR method for 6-, 14-, 57- and 118-bus IEEE systems, and a 582-bus real system are shown in Figure 3.6. It can be seen that the proposed method shows consistent monotonic convergence property for all the systems studied.

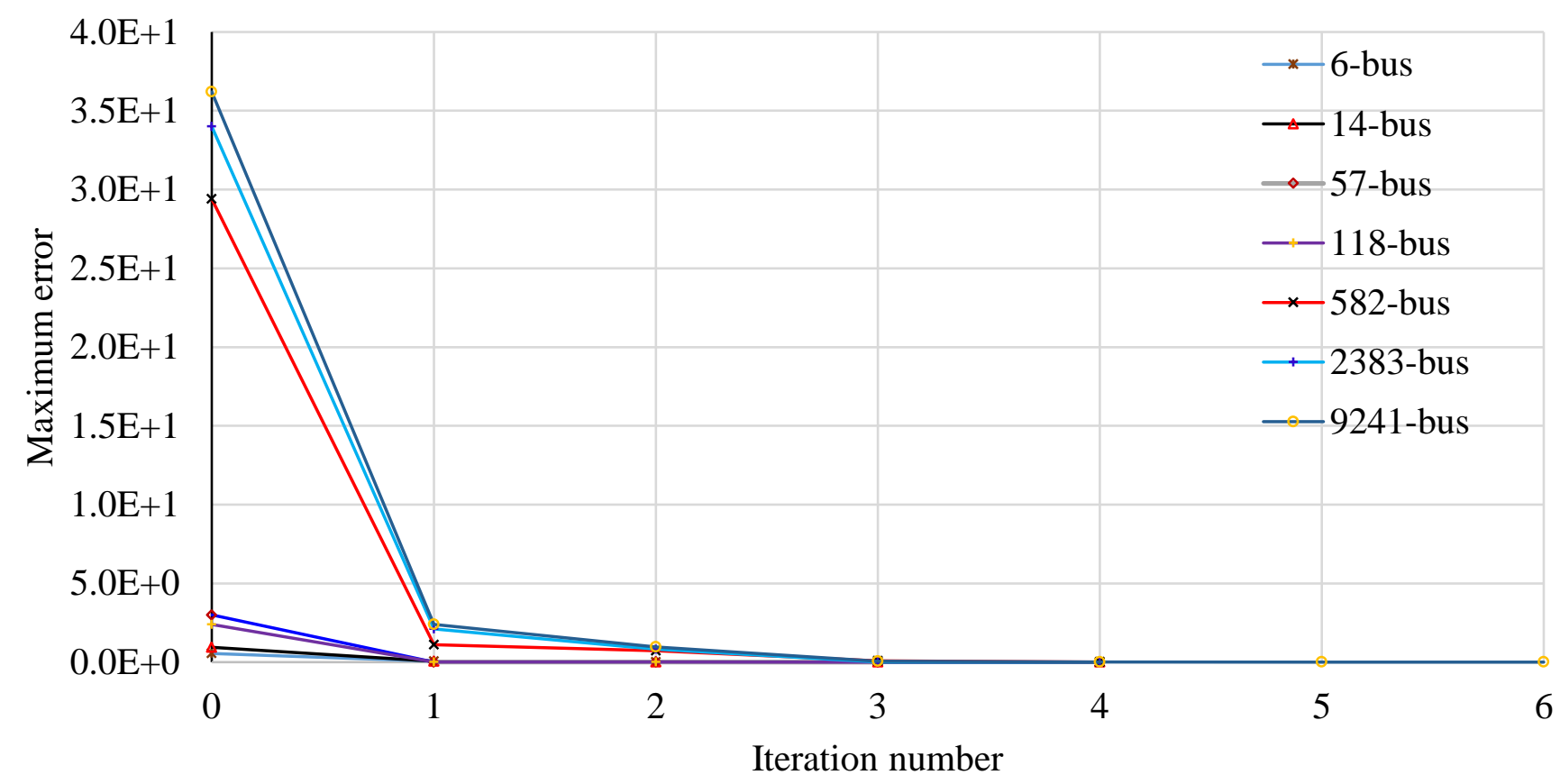

Figure 3.6 Convergence characteristic of the proposed method for different test systems.

Table 3.1 reports test results such as the number of iterations and the execution times for the proposed rectangular line-wise PF as well as the rectangular bus-wise NR methods at a tolerance of $10^{-5}$ per-unit. It can be noticed that the proposed rectangular line-wise NR method is computationally equal or up to thrice as fast as the rectangular bus-wise NR method. 
Table 3.1 Number of Iterations and Execution Times at $10^{-5}$ per unit tolerance

\begin{tabular}{|c|c|c|c|c|c|}
\hline \multirow{2}{*}{ System } & \multicolumn{2}{|c|}{$\begin{array}{c}\text { Traditional Rectangular bus- } \\
\text { wise NR }\end{array}$} & \multicolumn{2}{|c|}{$\begin{array}{c}\text { Proposed Rectangular line-wise } \\
\text { NR }\end{array}$} & \multirow{2}{*}{$\begin{array}{l}\text { Execution times of } \\
\text { rectangular line-wise } \\
\text { as percentage of } \\
\text { rectangular bus-wise }\end{array}$} \\
\hline & $\begin{array}{c}\text { Time } \\
\text { (m.seconds) }\end{array}$ & $\begin{array}{c}\text { No. of } \\
\text { Iterations }\end{array}$ & $\begin{array}{c}\text { Time } \\
\text { (m.seconds) }\end{array}$ & No. of Iterations & \\
\hline IEEE 6-bus & 6.0 & 3 & 4.0 & 4 & $66.67 \%$ \\
\hline IEEE 14-bus & 9.0 & 2 & 5.0 & 3 & $55.56 \%$ \\
\hline IEEE 57-bus & 24.0 & 3 & 8.0 & 3 & $33.33 \%$ \\
\hline IEEE 118-bus & 35.0 & 3 & 14.0 & 4 & $40.00 \%$ \\
\hline Real 582-bus & 338.0 & 6 & 139.0 & 4 & $41.12 \%$ \\
\hline POLISH 2383-bus & 1592.0 & 4 & 936.0 & 4 & $58.79 \%$ \\
\hline PEGASE 9241-bus & 4815.4 & 6 & 2618.2 & 6 & $54.37 \%$ \\
\hline
\end{tabular}

\subsection{Discussion on Power Flow}

The proposed rectangular line-wise NR method for PF is consistently equal or faster than bus-wise NR method, up to triple the speed. To elaborate, a comparison is drawn between the number of terms in each formulation of rectangular line-wise NR and rectangular bus-wise NR algorithms in Table 3.2. Nevertheless, the number of equations of the proposed rectangular line-wise PF is higher than that of traditional rectangular bus-wise PF. It can be noticed that the proposed rectangular line-wise NR method has a lower number of products computed each time the mismatch vector is computed comparing to the high number of products of rectangular bus-wise PF, which is 170 times on average base (lowest was 1.3 times and highest was 828.4 times) the products of proposed method. Further, almost all the terms of rectangular bus-wise PF method are cubic. However, in the case of the proposed rectangular line-wise NR method, there are no cubic terms, only quadratic and linear terms. 
Table 3.2 Comparison of formulations of Rectangular Forms of Line-wise NR method and Bus-wise NR method for Non-zero Terms

\begin{tabular}{|c|c|c|c|c|c|c|c|}
\hline & \multirow[b]{2}{*}{ Method } & \multirow{2}{*}{$\begin{array}{c}\text { Number } \\
\text { Of } \\
\text { Equations }\end{array}$} & \multicolumn{3}{|c|}{ Number of terms } & \multirow{2}{*}{$\begin{array}{c}\text { Number of } \\
\text { Products }\end{array}$} & \multirow{2}{*}{$\begin{array}{c}\text { Ratio } \\
\text { between \# of } \\
\text { Products in } \\
\text { rectangular } \\
\text { bus-wise to } \\
\text { that of } \\
\text { rectangular } \\
\text { line-wise }\end{array}$} \\
\hline & & & Cubic Terms & $\begin{array}{l}\text { Quadratic } \\
\text { Terms }\end{array}$ & $\begin{array}{l}\text { Linear } \\
\text { Terms }\end{array}$ & & \\
\hline \multirow{2}{*}{$\begin{array}{l}\text { IEEE 6- } \\
\text { bus }\end{array}$} & $\begin{array}{c}\text { Rectangular } \\
\text { line-wise }\end{array}$ & 38 & 0 & 86 & 83 & 255 & \multirow{2}{*}{1.3} \\
\hline & $\begin{array}{c}\text { Rectangular } \\
\text { bus-wise }\end{array}$ & 10 & 99 & 2 & 0 & 321 & \\
\hline \multirow{2}{*}{$\begin{array}{l}\text { IEEE 14- } \\
\text { bus }\end{array}$} & $\begin{array}{c}\text { Rectangular } \\
\text { line-wise }\end{array}$ & 106 & 0 & 248 & 226 & 722 & \multirow{2}{*}{1.8} \\
\hline & \begin{tabular}{|c|}
$\begin{array}{c}\text { Rectangular } \\
\text { bus-wise }\end{array}$ \\
\end{tabular} & 26 & 418 & 8 & 0 & 1,322 & \\
\hline \multirow{2}{*}{$\begin{array}{l}\text { IEEE 57- } \\
\text { bus }\end{array}$} & $\begin{array}{c}\text { Rectangular } \\
\text { line-wise }\end{array}$ & 432 & 0 & 972 & 958 & 2,902 & \multirow{2}{*}{6.9} \\
\hline & $\begin{array}{c}\text { Rectangular } \\
\text { bus-wise }\end{array}$ & 112 & 6,572 & 12 & 0 & 19,964 & \\
\hline \multirow{2}{*}{$\begin{array}{c}\text { IEEE } \\
\text { 118-bus }\end{array}$} & $\begin{array}{c}\text { Rectangular } \\
\text { line-wise }\end{array}$ & 978 & 0 & 2,338 & 2,031 & 6,707 & \multirow{2}{*}{10.1} \\
\hline & $\begin{array}{c}\text { Rectangular } \\
\text { bus-wise }\end{array}$ & 234 & 22,263 & 106 & 0 & 67,469 & \\
\hline \multirow{2}{*}{$\begin{array}{c}\text { Real 582- } \\
\text { bus }\end{array}$} & $\begin{array}{c}\text { Rectangular } \\
\text { line-wise }\end{array}$ & 5,022 & 0 & 11,868 & 10,774 & 34,510 & \multirow{2}{*}{52.0} \\
\hline & $\begin{array}{c}\text { Rectangular } \\
\text { bus-wise }\end{array}$ & 1,162 & 597,566 & 288 & 0 & $1,795,598$ & \\
\hline \multirow{2}{*}{$\begin{array}{l}\text { POLISH } \\
\text { 2383-bus }\end{array}$} & $\begin{array}{c}\text { Rectangular } \\
\text { line-wise }\end{array}$ & 16,348 & 0 & 35404 & 36482 & 107,290 & \multirow{2}{*}{296.4} \\
\hline & $\begin{array}{c}\text { Rectangular } \\
\text { bus-wise }\end{array}$ & 4,764 & $10,597,944$ & 652 & 9,528 & $31,804,664$ & \\
\hline \multirow{2}{*}{$\begin{array}{l}\text { PEGASE } \\
\text { 9241-bus }\end{array}$} & $\begin{array}{c}\text { Rectangular } \\
\text { line-wise }\end{array}$ & 82,676 & 0 & 195,476 & 179,500 & 570,452 & \multirow{2}{*}{828.4} \\
\hline & $\begin{array}{c}\text { Rectangular } \\
\text { bus-wise }\end{array}$ & 18,480 & $157,514,856$ & 2,888 & 36,960 & $472,587,304$ & \\
\hline
\end{tabular}


On reviewing Table 3.3, it is clear that the number of products required to compute a Jacobian in the rectangular bus-wise NR method is about 266 times on average base (lowest was 1.2 times and highest was 1298.1 times) that of the line-wise NR method.

Table 3.3 Comparison of Jacobian Computation of Rectangular Forms of Line-wise and Bus-wise PF using Newton Raphson for Non-zero Terms

\begin{tabular}{|c|c|c|c|c|c|c|}
\hline & \multirow{2}{*}{ Method } & \multirow{2}{*}{$\begin{array}{c}\text { Number } \\
\text { of } \\
\text { Jacobian } \\
\text { Elements }\end{array}$} & \multicolumn{2}{|c|}{ Number of terms } & \multirow{2}{*}{$\begin{array}{c}\text { Number of } \\
\text { Products }\end{array}$} & \multirow{2}{*}{$\begin{array}{l}\text { Ratio between } \# \\
\text { of Products in } \\
\text { rectangular bus- } \\
\text { wise to that of } \\
\text { rectangular line- } \\
\text { wise }\end{array}$} \\
\hline & & & $\begin{array}{l}\text { Quadratic } \\
\text { Terms }\end{array}$ & Linear Terms & & \\
\hline \multirow{2}{*}{$\begin{array}{l}\text { IEEE 6- } \\
\text { bus }\end{array}$} & $\begin{array}{c}\text { Rectangular } \\
\text { line-wise }\end{array}$ & 170 & 24 & 128 & 246 & \multirow{2}{*}{1.2} \\
\hline & $\begin{array}{c}\text { Rectangular } \\
\text { bus-wise }\end{array}$ & 56 & 102 & 2 & 206 & \\
\hline \multirow{2}{*}{$\begin{array}{l}\text { IEEE 14- } \\
\text { bus }\end{array}$} & $\begin{array}{c}\text { Rectangular } \\
\text { line-wise }\end{array}$ & 519 & 56 & 568 & 680 & \multirow{2}{*}{2.0} \\
\hline & $\begin{array}{c}\text { Rectangular } \\
\text { bus-wise }\end{array}$ & 146 & 678 & 8 & 1,364 & \\
\hline \multirow{2}{*}{$\begin{array}{c}\text { IEEE 57- } \\
\text { bus }\end{array}$} & $\begin{array}{c}\text { Rectangular } \\
\text { line-wise }\end{array}$ & 2,179 & 228 & 2,252 & 2,708 & \multirow{2}{*}{9.3} \\
\hline & $\begin{array}{c}\text { Rectangular } \\
\text { bus-wise }\end{array}$ & 772 & 12,546 & 12 & 25,104 & \\
\hline \multirow{2}{*}{$\begin{array}{c}\text { IEEE } \\
\text { 118-bus }\end{array}$} & $\begin{array}{c}\text { Rectangular } \\
\text { line-wise }\end{array}$ & 5,127 & 472 & 5,314 & 6,258 & \multirow{2}{*}{17.5} \\
\hline & $\begin{array}{c}\text { Rectangular } \\
\text { bus-wise }\end{array}$ & 1,484 & 54,758 & 106 & 109,622 & \\
\hline \multirow{2}{*}{$\begin{array}{c}\text { Real } 582- \\
\text { bus }\end{array}$} & $\begin{array}{c}\text { Rectangular } \\
\text { line-wise }\end{array}$ & 20,916 & 2,328 & 27,308 & 31,964 & \multirow{2}{*}{84.5} \\
\hline & $\begin{array}{c}\text { Rectangular } \\
\text { bus-wise }\end{array}$ & 6,559 & $1,350,246$ & 288 & $2,700,780$ & \\
\hline \multirow{2}{*}{$\begin{array}{l}\text { POLISH } \\
\text { 2383-bus }\end{array}$} & $\begin{array}{c}\text { Rectangular } \\
\text { line-wise }\end{array}$ & 5,127 & 9,532 & 81,740 & 100,804 & \multirow{2}{*}{450.3} \\
\hline & $\begin{array}{c}\text { Rectangular } \\
\text { bus-wise }\end{array}$ & 1,484 & $22,695,698$ & 652 & $45,392,048$ & \\
\hline \multirow{2}{*}{$\begin{array}{l}\text { PEGASE } \\
\text { 9241-bus }\end{array}$} & $\begin{array}{c}\text { Rectangular } \\
\text { line-wise }\end{array}$ & 5,127 & 36964 & 452,260 & 526,188 & \multirow{2}{*}{1298.1} \\
\hline & $\begin{array}{c}\text { Rectangular } \\
\text { bus-wise }\end{array}$ & 1,484 & $341,510,402$ & 2,888 & $683,023,692$ & \\
\hline
\end{tabular}


These aspects make the computation of Jacobian for the line-wise NR method much faster in comparison with the bus-wise NR method. In an iterative algorithm, such as the PF analysis, these differences add up and make the line-wise NR method faster than the bus-wise NR method.

Further, to compare the characteristics of the Jacobian matrices of the line-wise NR method and the bus-wise NR method, the Condition number and Sparsity factors are reported in Table 3.4. The dimension of the Jacobian matrix of the proposed rectangular line-wise NR method is computed using details provided at the end of section 3.3, where the total number of equations and unknowns equal $(4 \cdot N T+2 \cdot N B-2)$. Condition numbers of both rectangular line-wise NR and bus-wise NR methods are well-behaved. Sparsity factors also remain similar. Finally, it can be seen than the line-wise NR method scales very well for larger systems without any difficulty and hence is suitable for commercial-grade applications.

Table 3.4 Comparison of Size and Condition Number of Jacobian Matrix for Both Rectangular Forms of Linewise NR method and Bus-wise NR method

\begin{tabular}{|c|c|c|c|c|c|c|}
\hline & \multicolumn{3}{|c|}{$\begin{array}{l}\text { Traditional rectangular bus-wise Jacobian } \\
\text { matrix }\end{array}$} & \multicolumn{3}{|c|}{$\begin{array}{l}\text { Proposed rectangular line-wise Jacobian } \\
\text { matrix }\end{array}$} \\
\hline & Size & $\begin{array}{c}\text { Condition } \\
\text { Number } \\
\end{array}$ & $\begin{array}{l}\text { Sparsity } \\
\text { Factor }\end{array}$ & Size & $\begin{array}{c}\text { Condition } \\
\text { Number }\end{array}$ & $\begin{array}{l}\text { Sparsity } \\
\text { Factor }\end{array}$ \\
\hline IEEE 6-bus & $10 \times 10$ & $2.85 \cdot 10^{1}$ & 44 & $38 \times 38$ & $6.68 \cdot 10^{1}$ & 88.23 \\
\hline IEEE 14-bus & $26 \times 26$ & $7.01 \cdot 10^{2}$ & 78.40 & $106 \times 106$ & $2.78 \cdot 10^{2}$ & 95.38 \\
\hline IEEE 57-bus & $112 \times 112$ & $7.01 \cdot 10^{2}$ & 93.85 & $432 \times 432$ & $1.24 \cdot 10^{3}$ & 98.83 \\
\hline IEEE 118-bus & $234 \times 234$ & $3.01 \cdot 10^{3}$ & 97.29 & $978 \times 978$ & $4.55 \cdot 10^{3}$ & 99.46 \\
\hline Real 582-bus & $1,162 \times 1,162$ & $4.60 \cdot 10^{6}$ & 99.51 & $5,022 \times 5,022$ & $8.9 \cdot 10^{5}$ & 99.92 \\
\hline POLISH 2383-bus & $4,764 \times 4,764$ & $7.9 \cdot 10^{7}$ & 99.88 & $16,348 \times 16,348$ & $4.2 \cdot 10^{7}$ & 99.98 \\
\hline $\begin{array}{l}\text { PEGASE 9241- } \\
\text { bus }\end{array}$ & $18,480 \times 18,480$ & $8.6 \cdot 10^{8}$ & 99.94 & 82,676 X 82,676 & $3.4 \cdot 10^{8}$ & 99.99 \\
\hline
\end{tabular}




\subsection{Benefits}

As discussed in Section 2.8, LWPF can complete power flow analysis in a reduced amount of time. While the fastest is up to three times that of BWPF formulation, it is shown to be twice as fast for larger systems, as reported Table 3.1. Accordingly, as described in Section 2.8, power flow analysis has very real and practical applications such as contingency analysis in operating power systems. As reported in Section 2.8, using the proposed rectangular formulation of LWPF, these practical benefits can be realized.

\subsection{Summary}

Rectangular bus-wise power balance equations and their solution based on the NR method and its variants are numerically proven as powerful PF analysis tools, possessing high degree of accuracy and speed.

In this chapter, a new line-wise set of power balance equations in rectangular form and its solution using NR technique are proposed. The formulation uses real and imaginary terms of bus voltage. It mainly considers the voltage difference for the transmission branches.

The proposed line-wise NR method is tested and compared for performance with the bus-wise NR method on 6-, 14-, 57- and 118-bus IEEE systems, a 582-bus real power system, a 2383-bus Polish power system, and a 9241-bus PEGASE system. As usual in rectangular coordinates, the use of real and imaginary terms of voltage leads to elimination of trigonometric functions, i.e. sine and cosine terms. Coupled with this fact and the use of real and reactive line-wise power flows as variables, the set of rectangular line-wise equations and its solution using NR technique is faster for all tested systems, up to three times, compared with bus-wise NR method. Numerical analysis of the Jacobians of bus-wise and line-wise NR methods shows that the number of calculations to be completed by rectangular line-wise NR method is much lower, resulting in the higher speed of the rectangular line-wise NR method.

The rectangular line-wise NR method reveals a stable numerical performance, monotonic convergence and solution accuracy. It scales well, as shown, for larger systems. The active and reactive line power flows result directly from the solution with no further need for computation. The necessity to construct the system admittance matrix is eliminated. Development of the system Jacobian is straightforward and requires no knowledge of the system topology.

Summarizing, the advantages of the proposed rectangular line-wise NR method are:

(1) It is superior to the bus-wise power method as it solves faster up to three times. 
(2) It demonstrates a stable numerical performance, monotonic convergence, solution accuracy and scales well for larger systems.

(3) It provides active and reactive line power flows with no further need for computation.

(4) It does not need the system admittance matrix.

(5) Its system Jacobian construction is straightforward and requires no knowledge of the system topology. Further, almost all terms are linear. 


\section{Chapter Four}

\section{Chapter 4: Line-Wise Optimal PoWer Flow Using Successive LINEAR OPTIMIZATION TECHNIQUE}

Optimal power flow (OPF) is an important Power Systems Optimization tool. OPF optimizes a chosen objective and satisfies power balance equations amongst other equalities and inequalities. The set of buswise power balance equations are most popularly used in this exercise. However, due to its inherently nonlinear solution space, OPF may yield a local optimal solution leading to economic loss. For this reason, it remains a major research topic. Changing the set of power balance equations changes the solution space, and hence time and the chance of reaching a better solution. Line-wise power balance equations are shown to provide a faster solution, up to twice the speed of bus-wise power balance equations, and have a better solution space due to the use of square of voltage magnitudes.

In this chapter, a new simple line-wise optimal power flow (LWOPF) formulation is proposed using line-wise power balance equations and solved using successive linear programming (SLP) technique. The study results on nine systems, up to one of 9,000 busses, show that the proposed method is accurate, provides monotonic convergence, scales well for large systems and is consistently faster, up to twice the speed of MATPOWER and other proven bus-wise SLP approaches to solve OPF.

\subsection{Introduction}

Optimal power flow (OPF) is an optimization tool that finds the best operating schedule to optimize power system operation considering an objective while satisfying power balance equations and other constraints. The objective function of OPF is formulated according to the objective of the study, such as minimizing the total generation cost. There are several important applications of the OPF tool, which cannot be enumerated exhaustively. A few important applications of the OPF tool include: (1) power system operators schedule their system at a frequent interval using the OPF tool, as often as every five minutes [1]; (2) dual variables of OPF formulation provide price signals for resources and equipment limits; (3) OPF and 
its variants are used as a basic tool for assessing various investment decisions; and; (4) OPF and its variants form a subset of formulations for unit commitment, optimal planning, and such other applications.

The reliability, robustness and relative speed of linear programming (LP) techniques make its approaches among the most extensively used methods to solve the OPF formulation in the literature [95]. As mentioned before in section 1.2.3, Successive linear programming (SLP) technique was employed for solving the power system scheduling problems in general and used as one of the most popular approaches for solving the OPF problem. The SLP approach basically involves three main steps.

Step 1: A set of power balance equations is solved (power flow) to compute current point for the power system.

Step 2: A linear incremental OPF model is developed at the current operating point and is solved using the chosen linear optimization technique to determine optimal change in the control vector.

Step 3: The control vector is updated using the optimal changes determined in Step 2.

The SLP algorithm repeats the set of steps 1,2 and 3 successively until a near optimal solution is computed based on the stopping criterion.

\subsubsection{Rationale for the developed LWOPF formulation}

In most cases, if not all, a power system is represented using a bus-wise set of power balance equations. While the bus-wise formulation is succinct in its representation, all its Jacobian terms contain a sinusoidal term and it is developed in terms of bus voltages. For large power systems, particularly for ill-conditioned systems, OPF is problematic, due to nonlinearity and the poor state of the Jacobian matrix.

In recent research developments, it is shown that a line-wise power flow formulation uses far fewer sinusoidal terms and is developed in terms of squares of bus voltage, resulting in lower order terms as shown in Chapter 2. Further, the polar line-wise power balance equations use square of the voltage magnitude instead of voltage magnitude itself. Using square of voltage magnitude brings in the benefit of having a linear relationship between power and square of voltage magnitude for impedance loads. Due to these reasons, it is shown that the line-wise power flow method is superior to the bus-wise power flow method and is up to twice as fast. Hence, it is hypothesized that by replacing bus-wise power balance equations by line-wise power balance equations in an OPF formulation, its speed will improve and also yield a better solution space due to the modeling using the square of voltage magnitudes. 
The main Contributions of developed LWOPF method

Benefitting from the improved speed and a better solution space of the polar line-wise power flow method reported in chapter two, a new OPF method is developed in this chapter. A line-wise OPF (LWOPF) formulation is proposed that uses a set of line-wise power balance equations. A linear incremental model of LWOPF formulation is created and solved using SLP technique. The main contributions of this work are summarized below:

1. A novel nonlinear OPF formulation based on a set of line-wise power balance equations is developed.

2. A linear incremental model of the nonlinear LWOPF model is developed.

3. Using a linear incremental model of LWOPF and SLP technique, a new OPF algorithm is developed and implemented.

LWOPF method is tested upon nine different systems from 6 busses to 9000 busses. Performance of LWOPF is compared with conventional bus-wise OPF (BWOPF). It establishes the superiority of the proposed LWOPF algorithm, as it converges up to two times faster and provides a better solution space, resulting in better optimal solutions. The speed of the proposed method is due to the lower order and simpler terms, and better solution is due to the improved solution space.

\subsection{Line-wise Optimal Power Flow Formulation}

This section details the development of the line-wise optimal power flow (LWOPF) based on a set of line-wise power balance equations.

The typical OPF can be expressed as in (4.1) - (4.3)

$$
\min f o(\boldsymbol{W})
$$

subject to:

$$
\begin{aligned}
& f g(\boldsymbol{W})=d \\
& \underline{h} \leq f h(\boldsymbol{W}) \leq \bar{h}
\end{aligned}
$$


where $\boldsymbol{W}$ is the problem vector. The function $f o(\boldsymbol{W})$ is the objective function in (1). The functions $f g(\boldsymbol{W})$ and $f h(\boldsymbol{W})$ are the set of functions for equality and inequality constraints respectively. In (4.2), $d$ is the target vector. In (4.3), $\underline{h}$ and $\bar{h}$ are the lower and upper limits on the inequality constraint.

\subsubsection{Objective function}

The optimization problem is stated in terms of the problem vector $\boldsymbol{W}=[U, \delta, P F, P S, Q F, Q S, P G, Q G]^{T}$, and comprises control and dependent vectors of $\boldsymbol{W C}=[U, P G]^{T}$ and $\boldsymbol{W D}=[\delta, P F, P S, Q F, Q S, Q G]^{T}$ respectively.

Based on the available data for the considered tested systems, the study uses the second-order generation cost function as follows. The proposed method can be easily adapted to other nonlinear continuously differential objective functions as well.

$$
f o(\boldsymbol{W})=\sum_{g=1}^{N G} c 1_{g}+c 2_{g} \cdot P G_{g}+c 3_{g} \cdot P G_{g}{ }^{2}
$$

\subsubsection{Equality constraints}

As reported in section 2.2, the following set of equations comprise the line-wise power balance equations, (4.5) to (4.10), and can model the entire power system. This set of equations will be used in the proposed OPF formulation to model power balance equality constraints.

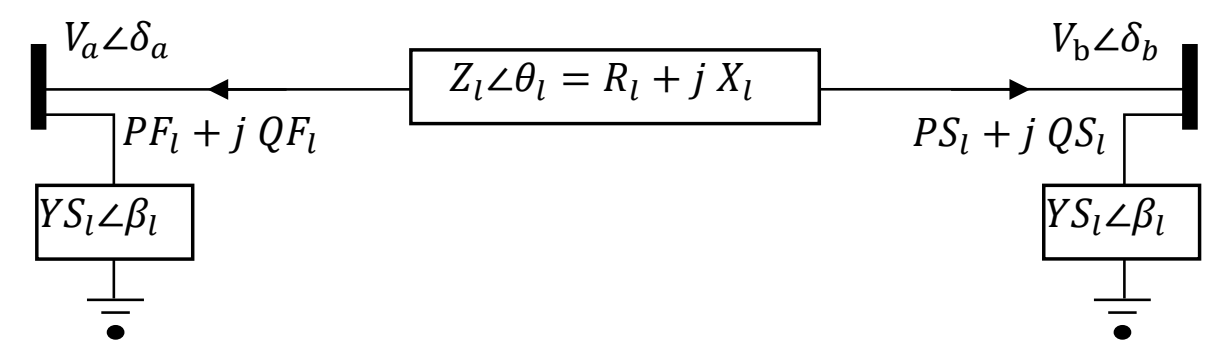

Figure 4.1 Series impedance element of the pi-model of the $l^{\text {th }}$ transmission branch connecting buses $a$ and $b$.

Considering a pi-model of a transmission line as shown in Figure 4.1, the square of sending and receiving end voltages may be related as below in (4.5) and (4.6). A detailed derivation is provided in Chapter 2. 


$$
\begin{array}{ll}
F F(\boldsymbol{W})=U_{a}{ }^{2}+2 U_{a} \cdot\left(P F_{l} \cdot R_{l}+Q F_{l} \cdot X_{l}-\frac{U_{b}}{2}\right)+S F_{l}{ }^{2} \cdot Z_{l}{ }^{2}=0 & \forall l=1 \text { to } N T \\
F S(\boldsymbol{W})=U_{b}{ }^{2}+2 U_{b} \cdot\left(P S_{l} \cdot R_{l}+Q S_{l} \cdot X_{l}-\frac{U_{a}}{2}\right)+S S_{l}{ }^{2} \cdot Z_{l}{ }^{2}=0 & \forall l=1 \text { to NT }
\end{array}
$$

Considering the phase angles of the sending and receiving end voltages, the relationships in (4.7) and (4.8) may be written:

$$
\begin{array}{ll}
F A(\boldsymbol{W})=\left(P F_{l} \cdot R_{l}+Q F_{l} \cdot X_{l}+U_{a}\right) \cdot \tan \left(\delta_{b}-\delta_{a}\right)-P F_{l} \cdot X_{l}+Q F_{l} \cdot R_{l}=0 & \forall l=1 \text { to NT } \\
F B(\boldsymbol{W})=\left(P S_{l} \cdot R_{l}+Q S_{l} \cdot X_{l}+U_{b}\right) \cdot \tan \left(\delta_{a}-\delta_{b}\right)-P S_{l} \cdot X_{l}+Q S_{l} \cdot R_{l}=0 & \forall l=1 \text { to NT }
\end{array}
$$

Further, the bus-wise power balance equations can be written in a matrix form as below:

$$
\begin{aligned}
& F P(\boldsymbol{W})=P G+[M]\left[\begin{array}{l}
P F \\
P S
\end{array}\right]-U \cdot G S=P D \\
& F Q(\boldsymbol{W})=Q G+[M]\left[\begin{array}{l}
Q F \\
Q S
\end{array}\right]+U \cdot B S=Q D
\end{aligned}
$$

where the shunt elements of lines in the pi-model and shunt loads are included in the GS and BS terms. Further, the constant loads are modeled in PD and QD in (4.9) and (4.10) respectively.

\subsubsection{Inequality constraints}

The limits on the control variables are as follows.

- Limits on Bus Voltages and Generators outputs are as below:

$$
\begin{array}{cc}
\underline{U_{i}} \leq U_{i} \leq \overline{U_{i}} & \forall i \in N B \\
\underline{P G_{g}} \leq P G_{g} \leq \overline{P G_{g}} & \forall g \in N G \\
\underline{Q G_{g}} \leq Q G_{g} \leq \overline{Q G_{g}} & \forall g \in N G
\end{array}
$$


Limits on MVA line power flows are as below:

$$
\begin{array}{ll}
P F_{l}{ }^{2}+Q F_{l}{ }^{2} \leq\left(\overline{S L_{l}}\right)^{2} & \forall l \in N T \\
P S_{l}{ }^{2}+Q S_{l}{ }^{2} \leq\left(\overline{S L_{l}}\right)^{2} & \forall l \in N T
\end{array}
$$

\subsubsection{Generic form of nonlinear LWOPF optimization formulation}

Succinctly, the formulation (4.4) - (4.13) can be stated below.

Using (4.4), the objective is:

$$
\min : f o(\boldsymbol{W})=\sum_{g=1}^{N G} c 1_{g}+c 2_{g} \cdot P G_{g}+c 3_{g} \cdot P G_{g}{ }^{2}
$$

subject to:

the equality constraints in $(4.5)-(4.10)$ are stated as:

$$
f g(\boldsymbol{W})=d
$$

the inequality constraints in $(4.11)-(4.15)$ are stated as:

$$
\underline{h} \leq f h(\boldsymbol{W}) \leq \bar{h}
$$

In (4.15), the $d=\left[\begin{array}{llllll}0 & 0 & 0 & 0 & P D & Q D\end{array}\right]^{T}$. In (16), the $\bar{h}=\left[\begin{array}{ll}\bar{U} \overline{P G} & \overline{Q G}\left(\overline{S L_{l}}\right)^{2}\left(\overline{S L_{l}}\right)^{2}\end{array}\right]^{T}$ and $\underline{h}=$ $\left[\begin{array}{llll}\underline{U} \underline{P G} & \underline{Q G} & 0 & 0\end{array}\right]^{T}$

The LWOPF formulation (4.16) - (4.18) is a nonlinear optimization formulation. It can be solved using SLP technique as described below. 


\subsection{Incremental Formulation for SLP Method}

The optimization problem stated in (4.16) to (4.18) can be solved by SLP technique using the following steps. The algorithm starts with the initial values of the control vector $\boldsymbol{W} \boldsymbol{C}^{\circ}$.

- Step 1: Compute the state of the power system $\boldsymbol{W}^{\circ}$ by solving the set of line-wise power balance equations (4.17) considering current values of the control vector $\boldsymbol{W} \boldsymbol{C}^{\circ}$.

- Step 2: Linearize the objective function (4.16) and set of nonlinear constraints (4.17) - (4.18) around an operating point $\boldsymbol{W}^{\circ}$. Set up the linear optimization formulation and solve to determine optimal incremental changes $\Delta \boldsymbol{W}$.

- Step 3: Update the problem vector $\boldsymbol{W}$ and go to step (1) successively until convergence is reached based on the stopping criterion.

In step 2, an incremental model is required and it is presented below. The problem vector $\Delta \boldsymbol{W}$ comprises control and dependent vectors of $\quad \Delta \boldsymbol{W}=[\Delta U, \Delta P G]^{T}$ and $\Delta \boldsymbol{W D}=[\Delta \delta, \Delta P F, \Delta P S, \Delta Q F, \Delta Q S, \Delta Q G]^{T}$ respectively. The linearized problem vector will be: $\Delta \boldsymbol{W}=[\Delta U, \Delta \delta, \Delta P F, \Delta P S, \Delta Q F, \Delta Q S, \Delta P G, \Delta Q G]^{T}$.

\subsubsection{The linearized objective function}

The linearized objective function from (4.16) will be as follows.

or

$$
\min \Delta f o(\boldsymbol{W})=\left[\frac{d f o(\boldsymbol{W})}{d \boldsymbol{W}}\right] \cdot \Delta \boldsymbol{W}
$$

$$
\min \Delta f o(\Delta \boldsymbol{W})=\sum_{g=1}^{N G}\left[\left(c 2_{g}+2 \cdot c 3_{g} \cdot P G_{g}\right) \cdot \Delta P G_{g}\right]
$$

\subsubsection{The linearized set of equality constraints}

The objective of the incremental formulation is subject to the following constraints. The linearized set of equality constraints (4.17) is as below:

$$
\Delta \mathrm{f} g(\boldsymbol{W})=\left[\begin{array}{ll}
\boldsymbol{J} & \boldsymbol{D}
\end{array}\right] \Delta \boldsymbol{W}=\Delta d
$$


where $\boldsymbol{J}$ is the Jacobian matrix of the set of line-wise power balance equations (4.17), as derived in Chapter 2 section 2.4, and shown below in (4.21), and $\boldsymbol{D}$ is a submatrix includes two vectors for the partial differentiation of the set (4.5) to (4.10) with respect to $P G$ and $Q G$ respectively as follows in (22). More details on the terms of (4.20) are available in Appendix A.

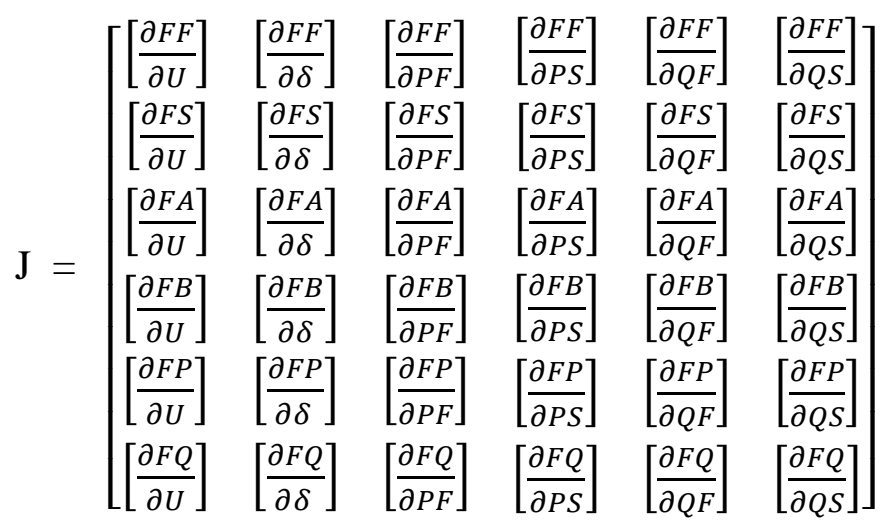

$$
\begin{aligned}
& D=\left[\begin{array}{llllll}
\frac{\partial F F}{\partial P_{G}} & \frac{\partial F S}{\partial P_{G}} & \frac{\partial F A}{\partial P_{G}} & \frac{\partial F A}{\partial P_{G}} & \frac{\partial F P}{\partial P_{G}} & \frac{\partial F Q}{\partial P_{G}} \\
\frac{\partial F F}{\partial Q_{G}} & \frac{\partial F S}{\partial Q_{G}} & \frac{\partial F B}{\partial Q_{G}} & \frac{\partial F B}{\partial Q_{G}} & \frac{\partial F P}{\partial Q_{G}} & \frac{\partial F Q}{\partial Q_{G}}
\end{array}\right]^{T}
\end{aligned}
$$

The vector $\Delta d=\left[\begin{array}{llllll}0 & 0 & 0 & 0 & 0 & 0\end{array}\right]^{T}$

\subsubsection{The linearized set of inequality constraints}

Moreover, the set of limits on the control variables in (4.11) to (4.15) could be linearized as follows. Considering the current operating value $\boldsymbol{W}^{\circ}$ and a step size of $\alpha$ :

$$
\underline{\Delta U_{i}} \leq \Delta U_{i} \leq \overline{\Delta U_{i}} \quad \forall i \in N B
$$

where $\underline{\Delta U_{i}}=\alpha \cdot\left(\underline{U_{i}}-U_{i}^{0}\right)$ and $\overline{\Delta U_{i}}=\alpha \cdot\left(\overline{U_{i}}-U_{i}^{0}\right)$.

$$
\underline{\Delta P G_{g}} \leq \Delta P G_{g} \leq \overline{\Delta P G_{g}} \quad \forall g \in N G
$$


where $\underline{\Delta P G_{g}}=\alpha \cdot\left(\underline{P G_{g}}-P G_{g}{ }^{0}\right)$ and $\overline{\Delta P G_{g}}=\alpha \cdot\left(\overline{P G_{g}}-P G_{g}{ }^{0}\right)$.

$$
\underline{\Delta Q G_{g}} \leq \Delta Q G_{g} \leq \overline{\Delta Q G_{g}} \quad \forall g \in N G
$$

where $\underline{\Delta Q G_{g}}=\alpha \cdot\left(\underline{Q G_{g}}-Q G_{g}{ }^{0}\right)$ and $\overline{\Delta Q G_{g}}=\alpha \cdot\left(\overline{Q G_{g}}-Q G_{g}{ }^{0}\right)$.

$$
\begin{array}{ll}
2 \cdot P F_{l} \cdot \Delta P F_{l}+2 \cdot Q F_{l} \cdot \Delta Q F_{l} \leq \overline{\Delta S L_{l}} & \forall l \in N T \\
2 \cdot P S_{l} \cdot \Delta P S_{l}+2 \cdot Q S_{l} \cdot \Delta Q S_{l} \leq \overline{\Delta S L_{l}} & \forall l \in N T
\end{array}
$$

where $\overline{\Delta S L_{l}}=\alpha \cdot\left(\overline{S L_{l}}-S L_{l}^{0}\right)$.

\subsubsection{Generic form of linearized LWOPF optimization problem}

The linear optimization formulation for the LWOPF is completely described in (4.19) - (4.27), where the objective is (4.19) and constraints are in (4.20) - (4.27). This incremental LWOPF problem can be collated into a generic formulation as below:

From (4.19):

$$
\min \Delta f o(\boldsymbol{W})=C^{T} \cdot \Delta \boldsymbol{W}
$$

Subject to: from (4.20) - (4.27):

$$
\underline{b} \leq A \cdot \Delta \boldsymbol{W} \leq \bar{b}
$$

where equality constraints will have the same lower and upper limit values. It can be solved using any linear optimization method such as Simplex, interior point algorithm, etc. The proposed successive linear programming algorithm is presented in the next section.

\subsection{Successive Linear Programming Algorithm}

This section describes the proposed algorithm to solve the LWOPF formulation using SLP technique as shown in Figure 4.2. The algorithm includes two main steps in each iteration: first, solving the line-wise power flow; second, linearization of the nonlinear LWOPF formulation around the operating point obtained from the first step, then solving the resultant linear optimization problem using a chosen LP technique. The entire procedure, the two steps, will be repeated successively till convergence based on the stopping criterion 
is reached. It may be useful here to note that the algorithm to solve the set of polar line-wise power flow, the (4.5) to (4.10) formulation, was explained before in [64] based on Newton-Raphson method.

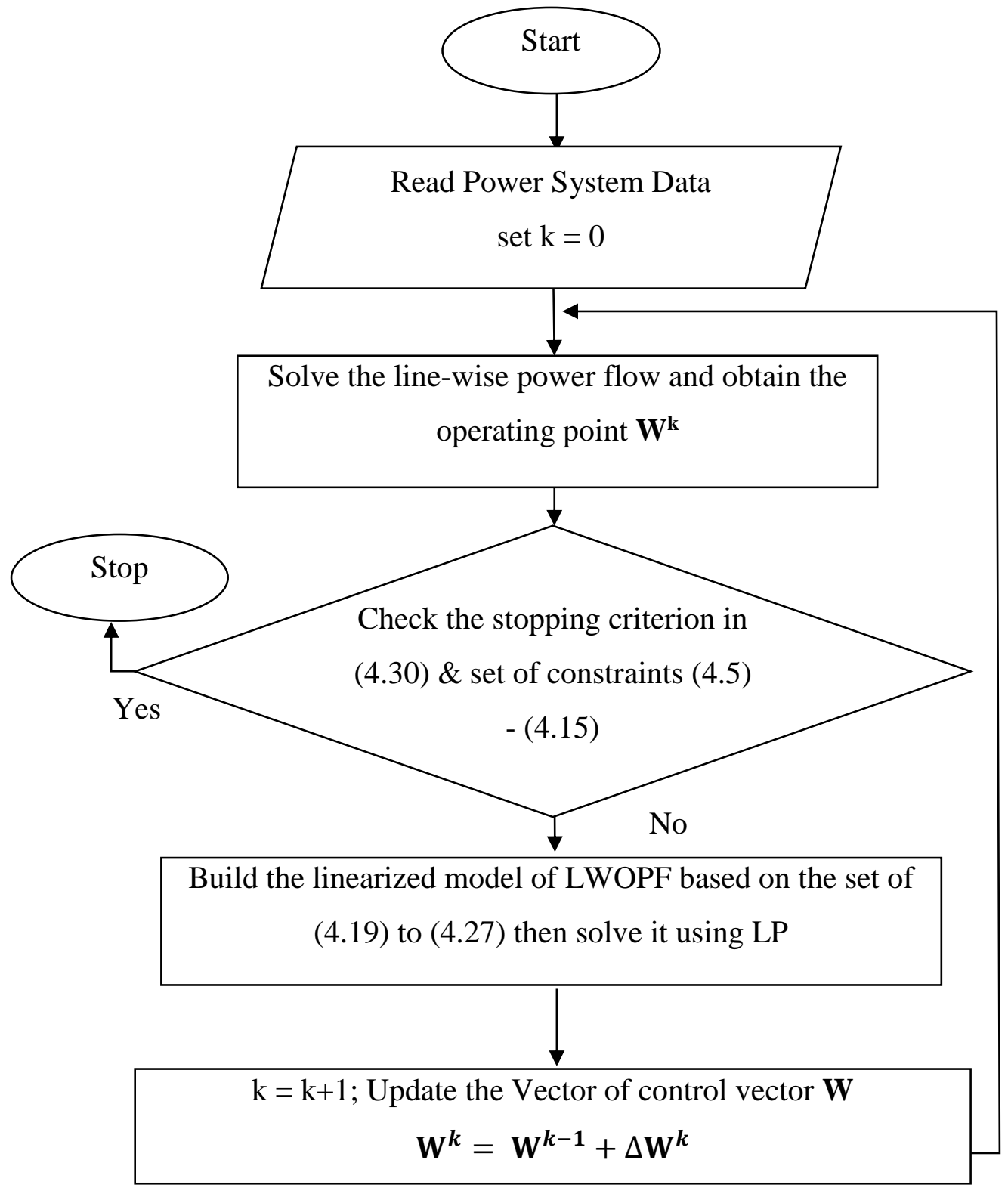

Figure 4.2 Flowchart of proposed LWOPF based on SLP.

The stopping criterion of the SLP algorithm is based on checking for the satisfaction of the set of constraints (4.5) - (4.15), then satisfying the condition in (4.30), or reaching the maximum number of iterations [104]. 


$$
\frac{\left|f o\left(\boldsymbol{W}^{k}\right)-f o\left(\boldsymbol{W}^{k-1}\right)\right|}{\left|f o\left(\boldsymbol{W}^{k}\right)\right|} \leq \gamma
$$

\subsection{Results of System Studies}

In this section, test results of the proposed line-wise OPF method are reported and compared with those from the bus-wise OPF method. The proposed algorithm and the SLP algorithm of the classical bus-wise method in [104] are implemented in the Matlab® R2016a environment and solved using the Mosek 7.0 solver. All tests are done on a 64-bit i7 Intel Core laptop (2.6 GHz, 16 GB of RAM) with the Windows 10 operating system. Sparse matrix implementations are considered where possible. The line flow limits for the tested power systems are obtained from [108]. In addition, the proposed mathematical formulation is implemented in AMPL [138] and solved using CPLEX [139] and GUROBI [140].

\subsubsection{Performance of the LWOPF method- Objective Function Value and Execution Time}

This subsection compares the OPF solutions of the MATPOWER (with default interior-point ACOPF solver (MIPS)) [65] and the proposed LWOPF based on SLP solution using three different solvers, MOSEK, CPLEX and GUROBI. The proposed SLP based LWOPF formulation considers the constraints of line flow limits. Table I presents results of these tests as percentage of results from MATPOWER. The proposed LWOPF-SLP method is tested on 6-, 14-, 30-, 57- and 118-bus IEEE systems, a 582-bus real power system, a 2383-bus Polish power system, and a 9241-bus PEGASE system.

It can be noticed from Table 4.1 that the proposed LWOPF obtains better solutions for all tested systems, namely lower generation costs than those obtained by MATPOWER. In terms of execution time, the proposed LWOPF method is up to twice as fast as MATPOWER. 
Table 4.1 Comparison of Proposed LWOPF Method using three solvers with MATPOWER - Objective Function Value and Execution Time

\begin{tabular}{c|c|c|c|c|c|c|c|c|c}
\hline \multirow{2}{*}{ System } & \multicolumn{3}{|c|}{ MOSEK } & \multicolumn{3}{c|}{ CPLEX } & \multicolumn{3}{|c}{ GUROUBI } \\
\cline { 2 - 10 } & $\begin{array}{c}\text { Time } \\
\text { ratio } \\
(\%)\end{array}$ & $\begin{array}{c}\text { Iter } \\
\#\end{array}$ & $\begin{array}{c}\text { Objective } \\
\text { function ratio } \\
(\%)\end{array}$ & $\begin{array}{c}\text { Time } \\
\text { ratio } \\
(\%)\end{array}$ & $\begin{array}{c}\text { Iter } \\
\#\end{array}$ & $\begin{array}{c}\text { Objective } \\
\text { function ratio } \\
(\%)\end{array}$ & $\begin{array}{c}\text { Time } \\
\text { ratio } \\
(\%)\end{array}$ & $\begin{array}{c}\text { Iter } \\
\#\end{array}$ & $\begin{array}{c}\text { Objective } \\
\text { function ratio } \\
(\%)\end{array}$ \\
\hline 6-bus & $40.6 \%$ & 2 & $98.56 \%$ & $47.8 \%$ & 2 & $99.99 \%$ & $53.6 \%$ & 2 & $98.56 \%$ \\
\hline 14-bus & $58.8 \%$ & 2 & $98.64 \%$ & $70.0 \%$ & 2 & $99.98 \%$ & $82.5 \%$ & 2 & $99.89 \%$ \\
\hline 30-bus & $49.0 \%$ & 2 & $98.3 \%$ & $49.0 \%$ & 2 & $99.84 \%$ & $52.0 \%$ & 2 & $99.62 \%$ \\
\hline 57-bus & $84.8 \%$ & 3 & $99.72 \%$ & $133.3 \%$ & 3 & $100.00 \%$ & $187.9 \%$ & 3 & $100.0 \%$ \\
\hline 118-bus & $42.5 \%$ & 4 & $97.58 \%$ & $45.7 \%$ & 4 & $99.81 \%$ & $53.3 \%$ & 4 & $97.14 \%$ \\
\hline $300-$-bus & $76.1 \%$ & 3 & $95.90 \%$ & $94.0 \%$ & 3 & $98.05 \%$ & $98.3 \%$ & 3 & $98.12 \%$ \\
\hline 582-bus & $82.1 \%$ & 4 & $97.77 \%$ & $85.3 \%$ & 4 & $100.00 \%$ & $85.3 \%$ & 4 & $97.95 \%$ \\
\hline 2383-bus & $56.4 \%$ & 3 & $99.46 \%$ & $65.1 \%$ & 3 & $99.98 \%$ & $65.1 \%$ & 3 & $99.48 \%$ \\
\hline 9241-bus & $62.3 \%$ & 6 & $97.52 \%$ & $111.5 \%$ & 6 & $99.84 \%$ & $154.5 \%$ & 6 & $99.86 \%$ \\
\hline
\end{tabular}

It is evident that the proposed LWOPF method using MOSEK solver is up to twice as fast as MATPOWER and consistently provides a better solution while satisfying all constraints. The reason for the improved solution is due to improved solution space as square of the voltage magnitude is used in the LWOPF formulation. The speed considerably improves due to simplicity of the LWOPF formulation and it is explained in section 4.6.

\subsubsection{Convergence characteristics of proposed algorithm}

This subsection presents the performance of the proposed LWOPF method as shown in Fig. 4.3. It can be noted that the proposed algorithm has a good monotonic convergence characteristic for different test systems at mismatch tolerance of $\gamma=10^{-6}$. The graphs in Fig. 4.3 are generated using the MOSEK solver since it performs the best as per Table 4.1. The Y-axis in Fig. 4.3 presents the deviation of the objective function, which refers to the difference between the value of the objective function determined by the proposed algorithm and the best-known value evaluated using MATPOWER, expressed as a percentage of the best-known value evaluated using MATPOWER.

From Figure 4.3, it is evident that irrespective of the system size, LWOPF provides monotonic convergence at up to twice the speed of MATPOWER while respecting all constraints. It is important to state that selection of step size influences performance of LWOPF method and it is acquired by experience for each system. 


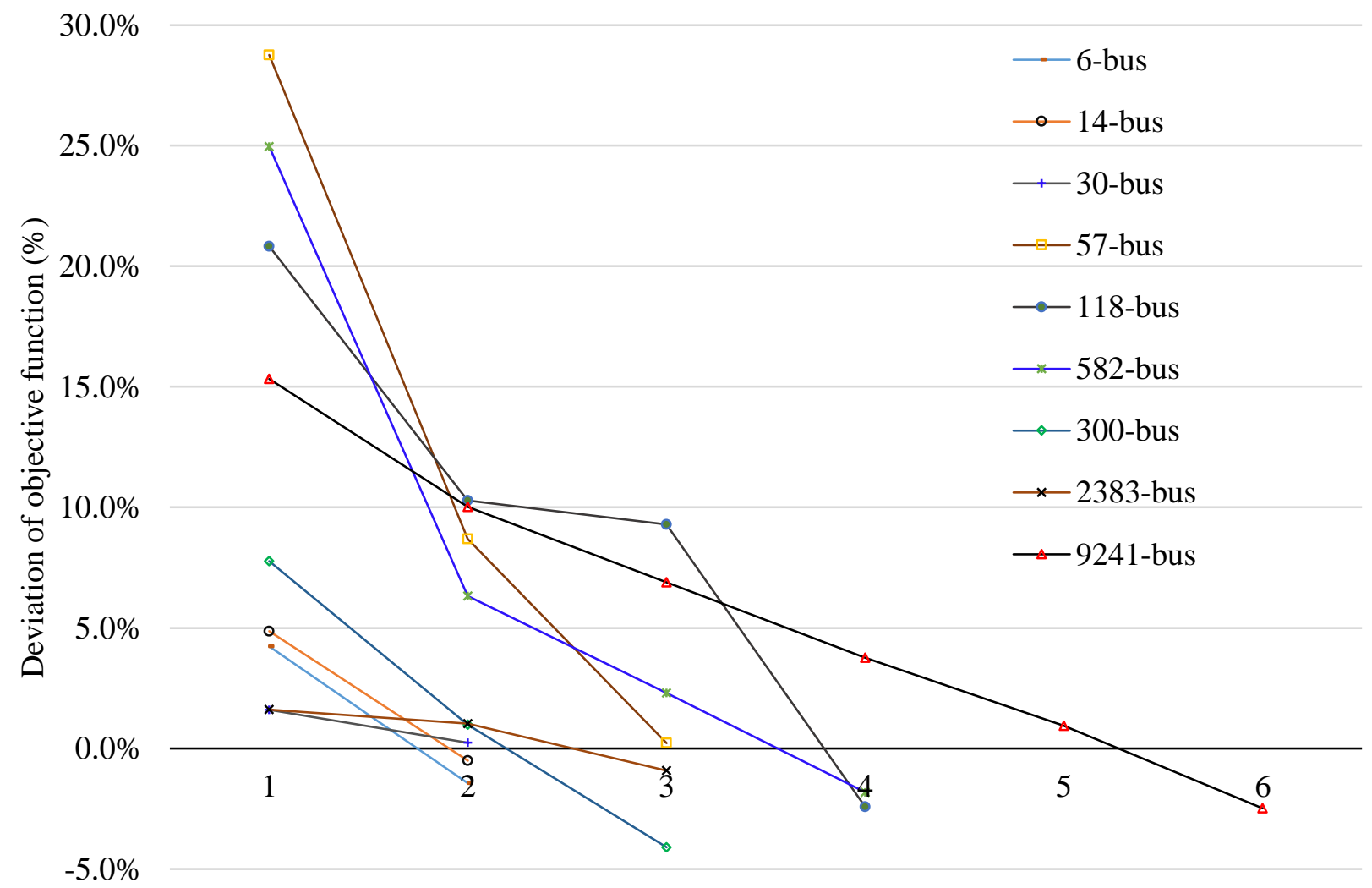

Iteration \#

Figure 4.3 Convergence characteristics of the proposed method for different test systems.

\subsubsection{Comparison between the proposed algorithm with an existing SLP algorithm of classical bus- wise method}

In this subsection, a comparison is developed between the results of the proposed SLP based LWOPF and the classical SLP based BWOPF [104]. The results of Table 4.2 show the superiority of the proposed formulation in terms of the solution quality, better objective function value, and faster execution time. In both of these implementations, same objective function, constraints (except for the set of power balance equations) and MOSEK solver was used to ensure that the comparison was fair.

From the results, it clear that the proposed method consistently provides a better solution in terms of objective function value. As before, it is because the solution space of the LWOPF method is simpler than that of the BWOPF method.

The reason for faster execution speed is primarily due to the simplicity of the LWOPF power balance equations and is better presented in Section 4.6. 
Table 4.2 Comparison between SLP based LWOPF and SLP Based BWOPF

\begin{tabular}{|c|c|c|c|c|c|c|c|c|}
\hline \multirow[b]{2}{*}{ 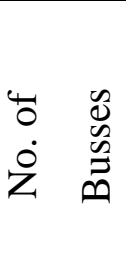 } & \multicolumn{2}{|c|}{ Objective: Generation Cost (\$) } & \multicolumn{4}{|c|}{ Execution Time (seconds) } & \multirow{2}{*}{$\begin{array}{c}\text { Objective } \\
\text { function ratio } \\
\text { LWOPF as \% } \\
\text { of BWOPF }\end{array}$} & \multirow{2}{*}{$\begin{array}{c}\text { Ratio of } \\
\text { execution times } \\
\text { LWOPF as \% } \\
\text { of BWOPF }\end{array}$} \\
\hline & $\begin{array}{c}\text { SLP Based } \\
\text { BWOPF }\end{array}$ & $\begin{array}{l}\text { SLP Based } \\
\text { LWOPF }\end{array}$ & $\begin{array}{c}\text { SLP Based } \\
\text { BWOPF }\end{array}$ & $\begin{array}{c}\text { Iter } \\
\#\end{array}$ & $\begin{array}{c}\text { SLP } \\
\text { Based } \\
\text { LWOPF }\end{array}$ & $\begin{array}{c}\text { Iter } \\
\#\end{array}$ & & \\
\hline 6 & $3,142.0$ & $3,094.7$ & 0.069 & 2 & 0.028 & 2 & $98.49 \%$ & $40.58 \%$ \\
\hline 14 & $5,198.9$ & $5,120.5$ & 0.080 & 2 & 0.047 & 2 & $98.64 \%$ & $58.75 \%$ \\
\hline 30 & 583.3 & 573.4 & 0.100 & 4 & 0.049 & 2 & $98.30 \%$ & $49.00 \%$ \\
\hline 57 & $41,760.0$ & $41,582.0$ & 0.165 & 4 & 0.140 & 3 & $99.57 \%$ & $84.85 \%$ \\
\hline 118 & $129,890.7$ & $126,521.0$ & 0.920 & 6 & 0.391 & 4 & $97.41 \%$ & $42.50 \%$ \\
\hline 300 & $719,753.6$ & $690,226.0$ & 1.170 & 4 & 0.890 & 3 & $95.90 \%$ & $76.07 \%$ \\
\hline 582 & $334,044.4$ & $326,596.0$ & 3.187 & 6 & 2.615 & 4 & $97.77 \%$ & $82.05 \%$ \\
\hline
\end{tabular}

\subsubsection{Sensitivity to step size}

This section shows the effects of step size on the solution quality in terms of convergence characteristics and the execution time to obtain the optimal solution. The Y-axis in Fig. 4.4 presents the deviation of the objective function from the best-known MATPOWER solution. It refers to the difference between the value of the objective function determined by the proposed algorithm and the best-known value evaluated using MATPOWER, expressed as a percentage of the best-known value calculated using MATPOWER.

A large step size makes the algorithm advance faster to the optimal solution but can result in oscillations when closer to the optimal solution, whereas a small step size will preclude oscillations but take more steps and hence more time to reach the optimal solution.

The step size in this work is the factor that scales the available margin for the control variables to move within the nonlinear bounds. It is defined by the value of $\alpha$ in (4.23) to (4.27). Hence, the step size in this work is a scalar.

Analysis is performed on the IEEE 118-bus power system. The convergence characteristics curves of the proposed LWOPF-SLP solution of the 118-bus system for various step sizes are shown in Fig. 4.4. It reports the deviation of the objective function resulting from the proposed LWOPF-SLP method at each iteration as a percentage compared to the best-known objective function value computed using 
MATPOWER. In the first couple of iterations, it can be observed that the solutions of the small step sizes, steps of 0.1 and 0.3 , are much better than those of the relatively large step sizes of 0.5 and 0.75 . At the step of 0.1 , an acceptable accurate solution can be obtained in only two iterations. On the other hand, it can be noticed that employment of the larger step sizes can yield better results. For instance, the final solution achieved by using a step size of 0.75 can yield a result that is better than the MATPOWER solution by almost $3 \%$ in the fourth iteration.

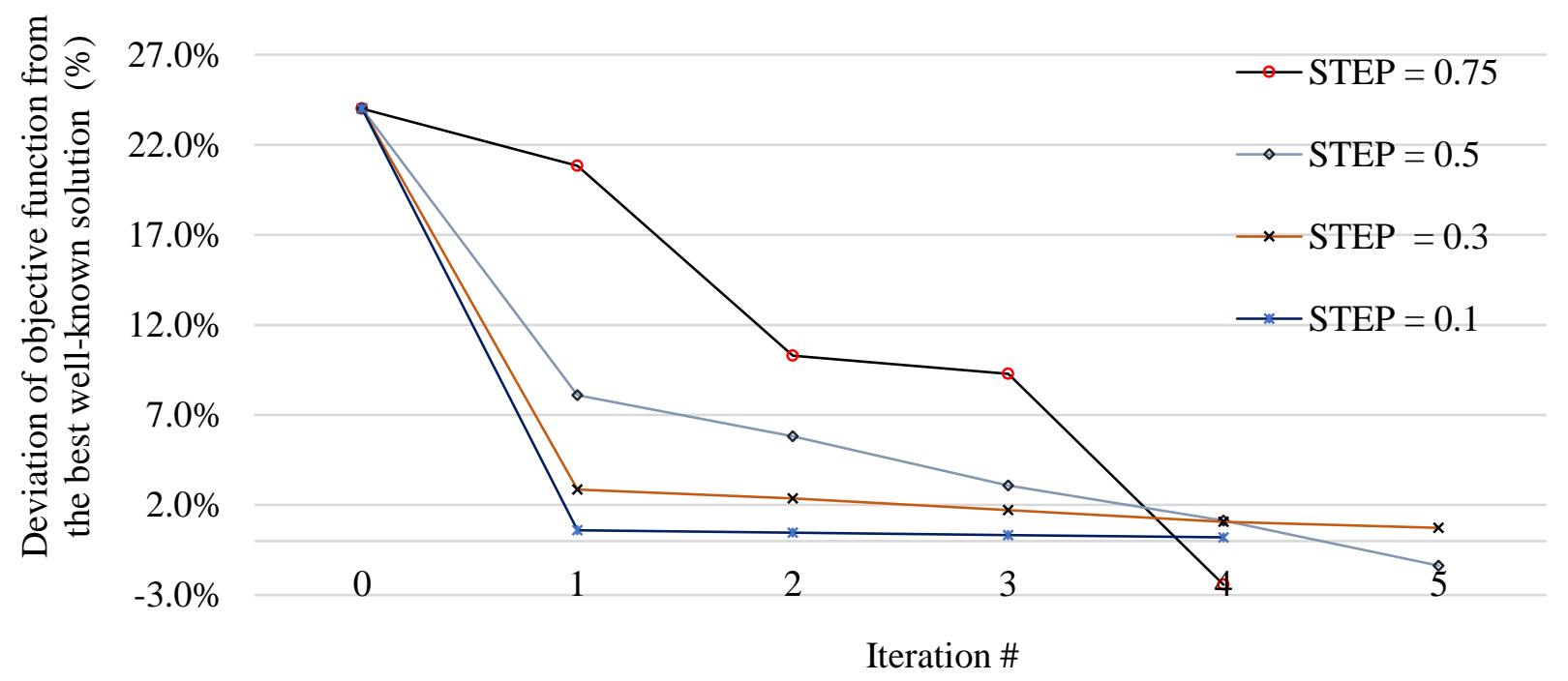

Figure 4.4 Convergence characteristics of proposed LWOPF-SLP solution for IEEE 118-bus power system for several step sizes.

\subsubsection{Sensitivity for load model}

In this subsection, several static load models are considered to investigate its effect on the solution performance of the proposed algorithm of LWOPF. Based on the two set of power balance equations of (4.8) and (4.9) the active and reactive demand power $P D$ and $Q D$ can be can be written as follows [29], [141].

- Exponential form:

$$
\begin{aligned}
& P D=P D_{0} \cdot\left(\frac{V}{V_{0}}\right)^{n p} \\
& Q D=Q D_{0} \cdot\left(\frac{V}{V_{0}}\right)^{n q}
\end{aligned}
$$


It can noted here that static constant power model is special case of ZIP model if $n p$ and $n q$ equal zero. It can be constant current if $n p$ and $n q$ equal one. It can be constant impedance if $n p$ and $n q$ equal two.

- Polynomial form (ZIP model):

$$
\begin{aligned}
& P D=P D_{0} \cdot\left[p_{1} \cdot\left(\frac{V}{V_{0}}\right)^{2}+p_{2}\left(\frac{V}{V_{0}}\right)+p_{3}\right] \\
& Q D=Q D_{0} \cdot\left[q_{1} \cdot\left(\frac{V}{V_{0}}\right)^{2}+q_{2}\left(\frac{V}{V_{0}}\right)+q_{3}\right]
\end{aligned}
$$

It can noted here that static constant power model is special case of ZIP model if $p_{1}, p_{2}, q_{1}$ and $q_{2}$ equal zero. It can be constant current if $p_{1}, p_{3}, q_{1}$ and $q_{3}$ equal zero. It can be constant impedance if $p_{2}, p_{3}, q_{2}$ and $q_{3}$ equal zero.

Each model of PD and QD is considered in concert. Each time, the Jacobian matrix in (4.21) is updated according to the considered model in (4.31) - (4.34). Three systems, 6-bus, 57-bus, and 118-bus systems, are tested to study the effects of load models on the performance of the proposed method, based on the available data of load models in [29] and [141]. Table 4.3 reports the results of these tests and compares performance of the proposed method for these load models. On reviewing Table 4.3, the performance of SLP based LWOPF is established to be consistent and reliable. It is not seen to be susceptible to changes in load models. 
Table 4.3 Performance Comparison between SLP based LWOPF Solutions with Various Load Models

\begin{tabular}{|c|c|c|c|c|}
\hline System & Load model & $\begin{array}{c}\text { Objective: } \\
\text { Generation } \\
\text { Cost }(\$)\end{array}$ & $\begin{array}{c}\text { Execution } \\
\text { time } \\
\text { (seconds) }\end{array}$ & $\begin{array}{c}\text { Iteration } \\
\#\end{array}$ \\
\hline \multirow{4}{*}{$\begin{array}{l}0 \\
0 \\
b\end{array}$} & Constant Power (CP) & $3,094.7$ & 0.028 & 2 \\
\hline & Constant Current (CC) & $3,094.7$ & 0.028 & 2 \\
\hline & Constant Impedance (CI) & $3,094.5$ & 0.029 & 2 \\
\hline & ZIP Model $(40 \% \mathrm{CP}, 30 \% \mathrm{CC}$ and $30 \% \mathrm{CI})$ & $3,095.2$ & 0.032 & 2 \\
\hline \multirow{4}{*}{$\frac{n}{0}$} & Constant Power (CP) & $4,1582.0$ & 0.140 & 3 \\
\hline & Constant Current (CC) & $4,1598.0$ & 0.144 & 3 \\
\hline & Constant Impedance (CI) & $4,1622.0$ & 0.149 & 3 \\
\hline & ZIP Model $(40 \% \mathrm{CP}, 30 \% \mathrm{CC}$ and $30 \% \mathrm{CI})$ & $4,1654.0$ & 0.152 & 3 \\
\hline \multirow{4}{*}{$\begin{array}{l}0 \\
0 \\
1 \\
0 \\
=\end{array}$} & Constant Power (CP) & $126,521.0$ & 0.391 & 4 \\
\hline & Constant Current (CC) & $126,578.0$ & 0.416 & 4 \\
\hline & Constant Impedance (CI) & $126,674.0$ & 0.428 & 4 \\
\hline & ZIP Model $(40 \% \mathrm{CP}, 30 \% \mathrm{CC}$ and $30 \% \mathrm{CI})$ & $126,988.0$ & 0.460 & 4 \\
\hline
\end{tabular}

\section{Error! Reference source not found.}

\subsubsection{Sensitivity for considering the LWPF solution in each iteration of SLP}

As mentioned before in section 4.4, the main loop of the proposed algorithm considers the solution line-wise PF (LWPF) in each iteration SLP. This section investigates the effects of considering or ignoring the step of LWPF solution on the performance of proposed algorithm. 
Table 4.4 Comparison between SLP based LWOPF with and without Considering the LWPF Step

\begin{tabular}{|c|c|c|c|c|c|}
\hline \multirow[b]{2}{*}{ 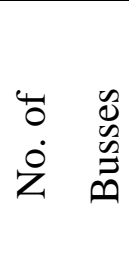 } & \multicolumn{4}{|c|}{ Execution Time (seconds) } & \multirow{2}{*}{$\begin{array}{l}\text { Ratio of execution } \\
\text { times } \\
\text { with LWPF \% and } \\
\text { without LWPF step }\end{array}$} \\
\hline & $\begin{array}{c}\text { SLP Based } \\
\text { LWOPF } \\
\text { with LWPF step }\end{array}$ & $\begin{array}{c}\text { Iteration } \\
\quad \#\end{array}$ & $\begin{array}{l}\text { SLP Based LWOPF } \\
\text { without LWPF step }\end{array}$ & $\begin{array}{c}\text { Iteration } \\
\quad \#\end{array}$ & \\
\hline 6 & 0.028 & 2 & 0.037 & 3 & $75.68 \%$ \\
\hline 14 & 0.047 & 2 & 0.056 & 3 & $80.36 \%$ \\
\hline 30 & 0.049 & 2 & 0.063 & 3 & $77.78 \%$ \\
\hline 57 & 0.140 & 3 & 0.151 & 4 & $92.72 \%$ \\
\hline 118 & 0.391 & 4 & 0.512 & 6 & $76.37 \%$ \\
\hline 300 & 0.890 & 3 & 1.1 & 4 & $80.91 \%$ \\
\hline 582 & 2.615 & 4 & 2.826 & 5 & $92.53 \%$ \\
\hline
\end{tabular}


Table 4.4 shows the comparison between the execution times and number iterations for the solution of proposed SLP based LWOPF with and without considering the LWPF calculation step. Considering the LWPF step could increase the execution time of each iteration. Nevertheless, it will reduce the total number of iterations and the total execution time.

\subsubsection{Sensitivity for initialization methods}

As mentioned before in section 4.4, the proposed algorithm considers the AC start based on line-wise $\mathrm{PF}$ as an initialization method before solving the SLP of LWOPF. In this section, two initialization methods: (1) flat start and (2) Line-wise power flow (LWPF) start are considered to show the effect of AC start. The analysis is done at different step sizes.

The ratio between the execution times of AC start to that of flat start is reported in Figure 4.5 for both 14- and 118-bus systems at several step size.

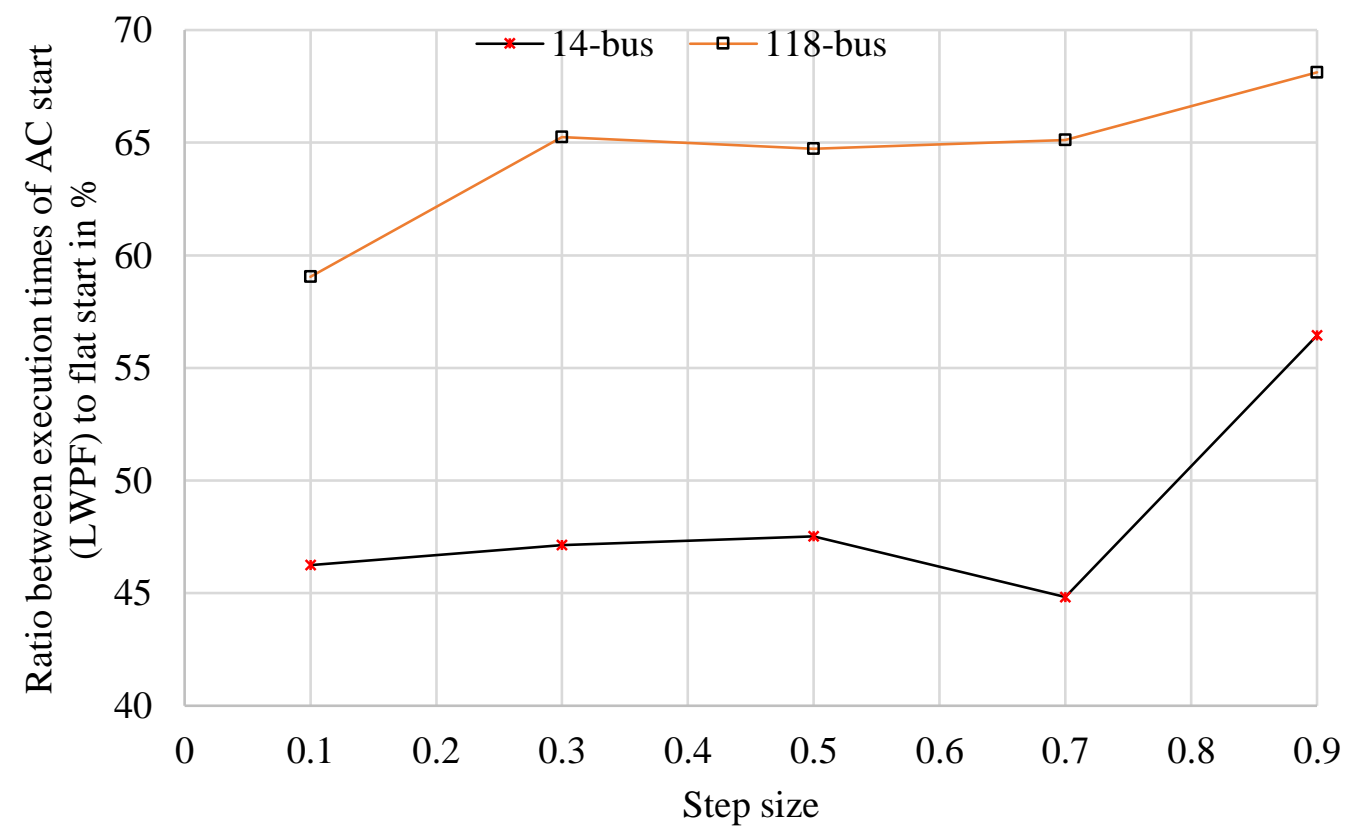

Figure 4.5 Ratio between execution times of LWPF start to flat start in percentage at different step sizes for both 14- and 118-bus systems. 


\subsection{Discussion on Optimal Power Flow}

The proposed solution algorithm of LWOPF which employs the SLP technique is consistently equal or faster than existing SLP algorithms of traditional BWOPF, up to twice the speed. The reasons for the faster speed of LWOPF are discussed here.

In order to compare and provide a numerical justification, Table 4.5 has been created, reporting on a number of relevant elements of the matrices in the formulation.

Upon analyzing Table 4.5, it is evident that LWOPF method has far fewer higher order terms in the A matrix and $\mathrm{C}$ vector compared with the BWOPF method. A higher order term is a product of two or more variables and/or constants. For example, a fourth order term, a higher order term, refers to the product of four variables and/or constants. Further, the number of sinusoidal terms in the A matrix is much less in the LWOPF method in comparison with the BWOPF method. Further, in comparing the A matrix for the two methods, it is evident that LWOPF method has less sinusoidal elements.

This significantly reduced need to compute sinusoidal terms immensely reduces the computational burden, making the LWOPF method much superior to the BWOPF method. 
Table 4.5 Comparison of SLP Formulations of SLP Based LWOPF and SLP Based BWOPF Algorithms

\begin{tabular}{|c|c|c|c|c|c|c|c|c|c|c|c|}
\hline \multirow{2}{*}{ 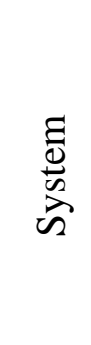 } & \multirow{2}{*}{ 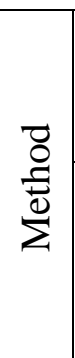 } & \multicolumn{2}{|c|}{ A Matrix } & \multicolumn{2}{|c|}{$\begin{array}{c}\text { Number of } \\
\text { Constraints in the } \\
\text { Problem }\end{array}$} & \multicolumn{4}{|c|}{$\begin{array}{c}\text { Number of higher order terms in } \\
\text { A matrix and } \mathrm{C} \text { vectors }\end{array}$} & \multicolumn{2}{|c|}{$\begin{array}{c}\text { Number of products } \\
\text { in A matrix and } \\
\text { C vectors }\end{array}$} \\
\hline & & Size & $\begin{array}{c}\text { Number } \\
\text { of } \\
\text { nonzero } \\
\text { terms }\end{array}$ & Equality & Inequality & 4th & $3 \mathrm{rd}$ & 2nd & 1 st & \begin{tabular}{|c|} 
With \\
Sine or \\
Cosine \\
Terms
\end{tabular} & $\begin{array}{l}\text { Without } \\
\text { Sine or } \\
\text { Cosine } \\
\text { Terms }\end{array}$ \\
\hline & & $40 \times 44$ & 140 & 40 & 10 & 0 & 0 & 56 & 340 & 56 & 396 \\
\hline & $\mathrm{BW}$ & $12 \times 16$ & 53 & 12 & 10 & 145 & 17 & 0 & 0 & 631 & 0 \\
\hline$+g$ & $\mathrm{LW}$ & $108 \times 118$ & 391 & 108 & 24 & 0 & 0 & 160 & 970 & 160 & 1,130 \\
\hline-5 & $\mathrm{BW}$ & $28 \times 38$ & 156 & 28 & 24 & 464 & 40 & 0 & 0 & 1,976 & 0 \\
\hline $0 \cong$ & LW & $224 \times 236$ & 917 & 224 & 42 & 0 & 0 & 328 & 1,980 & 328 & 2,308 \\
\hline$m=$ & BW & $60 \times 72$ & 373 & 60 & 42 & 958 & 101 & 0 & 0 & 4,135 & 0 \\
\hline$-g$ & $\mathrm{LW}$ & $434 \times 448$ & 1,692 & 434 & 71 & 0 & 0 & 640 & 3,854 & 640 & 4,494 \\
\hline & BW & $114 \times 128$ & 732 & 114 & 71 & 2,254 & 206 & 0 & 0 & 9,634 & 0 \\
\hline$\propto \cong$ & LW & $980 \times 1088$ & 3,418 & 980 & 226 & 0 & 0 & 1488 & 9,036 & 1,488 & 10,524 \\
\hline$=0$ & BW & $236 \times 344$ & 1,157 & 236 & 226 & 3,269 & 309 & 0 & 0 & 14,003 & 0 \\
\hline $8 \approx$ & LW & $2244 \times 2382$ & 9,465 & 2,244 & 438 & 0 & 0 & 3288 & 19,866 & 3,288 & 23,154 \\
\hline & $\mathrm{BW}$ & $600 \times 738$ & 3,874 & 600 & 438 & 7,867 & 992 & 0 & 0 & 34,444 & 0 \\
\hline$\approx \approx$ & $\mathrm{LW}$ & $5024 \times 5314$ & 21,206 & 5,024 & 872 & 0 & 0 & 7720 & 46,610 & 7,720 & 54,330 \\
\hline & $\mathrm{BW}$ & 1164 X 1454 & 7,030 & 1,164 & 872 & 21,284 & 1,892 & 0 & 0 & 90,812 & 0 \\
\hline$\infty \approx$ & $\mathrm{LW}$ & $16350 \times 17004$ & 57,047 & 16,350 & 3,037 & 0 & 0 & 23168 & 139,662 & 23,168 & 162,830 \\
\hline & $\mathrm{BW}$ & 44766 X 5420 & 28,437 & 4,766 & 3,037 & 87,147 & 7,727 & 0 & 0 & \begin{tabular}{|l|}
371,828 \\
\end{tabular} & 0 \\
\hline $\bar{\sigma} \approx$ & $\mathrm{LW}$ & $82678 \times 85568$ & 335,192 & 82,678 & 12,131 & 0 & 0 & 128,392 & 773,242 & \begin{tabular}{|l|}
128,392 \\
\end{tabular} & 901,634 \\
\hline న & $\mathrm{BW}$ & $18482 \times 21372$ & 131,579 & 18,482 & 12,131 & 337,947 & 30,041 & 0 & 0 & $1,441,911$ & 0 \\
\hline
\end{tabular}

Further, since after each LP solution, a power flow must be solved, the proposed method using the line-wise power flow is significantly faster compared to the bus-wise power flow method, adding to the speed of the proposed method as shown in Chapter 2. 


\subsection{Benefits}

The benefits of using the proposed LWOPF can be showcased with a real numerical example. The Ontario electricity market is used as an example. The benefits may be classified into technical and economic. Technical Benefit: To settle Ontario's electricity market, OPF is solved every five minutes. Using the proposed LWOPF method, assuming that the model for Ontario's power system has 300 busses and taking data from Table 4.2, the reduction in the time to optimize Ontario's electricity market is $25 \%$ (from 1.2 seconds to 0.9 seconds). Thus, LWOPF will enable Ontario's power system to operate at a better optimal state for 0.3 seconds, every five minutes (300 seconds).

Economic Benefit: Ontario’s electricity system distributed energy worth \$16,663,743,668 in the year 2017 as per the 2017 Yearbook of the Electricity Distributors Association of Ontario [142]. Referring to Table 4.2 and using the value of a $4 \%$ reduction in operating costs for a 300-bus system, the results produce an annual saving of $\$ 666,549,746$ or $\$ 76,090$ per hour for the province of Ontario. It is acknowledged that the amounts computed here include other charges in addition to the costs of the energy charges as well. However, it provides a measure for the potential benefit for this proposed LWOPF.

\subsection{Summary}

In this chapter, a new formulation for optimal power flow based on a set of line-wise power flow equations is proposed. The line-wise optimal power flow (LWOPF) formulation is linearized and solved using successive linear programming technique (SLP). The proposed LWOPF-SLP method is tested on 6-, 14-, 30-, 57- and 118-bus IEEE systems, a 582-bus real power system, a 2383-bus Polish power system, and a 9241-bus PEGASE system and compared for performance with traditional bus-wise OPF (BWOPF) implemented in MATPOWER and the best known SLP implementation in the literature.

Benefits: The LWOPF method demonstrates the benefits of

(1) stable numerical performance when scaled to large power systems,

(2) higher speed up to two times than that of BWOPF method,

(3) provides a monotonic convergence, and,

(4) a better optimal solution due to improved solution space modeling using square of voltage magnitudes. 
Contributions: Summarizing, the contributions of the proposed LWOPF-SLP method are:

(1) The developed method provides a novel and simple formulation for OPF based on the line-wise power flow formulation.

(2) Furthermore, it offers a simple SLP based solution algorithm for solving the proposed OPF formulation. The proposed LWOPF method, due to its simplicity in implementation, higher speed of execution, monotonic convergence characteristics, and, the ability to obtain a better optimal solution, it is well suited for commercial power systems application. 


\section{Chapter Five}

\section{ChaPTER 5: VOlTage STABiLITy CONSTRAined Line-WiSe OPTIMAL POWER FLOW}

In last chapter, first, a new simple line-wise optimal power flow (LWOPF) formulation was proposed based on line-wise power balance equations. Thereafter, a maximum loadability factor (MLF), as an indicator for voltage collapse, is derived and combined with the constraints of LWOPF to form a voltage stability constrained LWOPF (VSCLWOPF) model. As the line-wise power balance equations are based upon square of bus voltage magnitudes, it results in significant improvement in the solution space and lower order terms in all the computational steps. The purpose of this exercise is to examine the benefit of extending LWPF and LWOPF methods to VC and optimizing consider VC constraints.

The LWOPF and VSCLWOPF formulations, solved using nonlinear optimization technique, are tested on several IEEE benchmark systems as well as a real power system. The results show that the proposed LWOPF method is accurate, provides monotonic convergence, and scales well for large systems. It provides a better solution due to improved solution space and is consistently faster, up to twice the speed of MATPOWER, due to reduced computational needs. The results of VSCLWOPF shows that, for the same level of voltage stability, the solution costs less than that obtained by an OPF formulation with bus-wise power balance equations.

\subsection{Introduction}

In most cases, if not all, a power system is represented using a bus-wise set of power balance equations. While the bus-wise formulation is succinct in its representation, all its Jacobian terms contain a sinusoidal term and it is developed in terms of bus voltages. In Chapter 2, it is shown that a polar line-wise power flow formulation uses far fewer sinusoidal terms and is developed in terms of squares of bus voltage, resulting in lower order terms. Further, the polar line-wise power balance equations use square of the voltage magnitude instead of voltage magnitude itself. Using square of voltage magnitude brings in the benefit of having a linear relationship between power and square of voltage magnitude for impedance loads. Due to these reasons, it is shown that the line-wise power flow method is superior to the bus-wise power flow method and is up to twice as fast. Hence, it is hypothesized that by replacing bus-wise power balance equations with 
line-wise power balance equations in an OPF formulation, this will improve its speed and yield a better solution space due to the modelling using the square of voltage magnitudes. Moreover, it will meet the need to ensure the steady-state voltage stability of power system operation.

This chapter presents a novel and simple OPF algorithm based on the polar line-wise power balance formulation of Chapter 2. A maximum loadability factor is derived for branches and incorporated with the proposed Line-wise OPF (LWOPF) as a set voltage stability constraint. Two main issues are considered: first, the formulation of the proposed LWOPF and its performance in terms of solution space and execution time; and second, development of the maximum loadability factors of branches and how it could be incorporated with the proposed LWOPF. Both proposed formulations are tested on several power systems: 6-bus, 14-bus, 57-bus and 118-bus benchmark systems and the actual 582-bus system. The results show that the proposed method is accurate, provides monotonic convergence, scales well for large systems and is consistently faster, up to twice the speed of MATPOWER. The results of voltage stability constrained LWOPF shows the impacts of MLF on the objective cost function.

\subsection{Line-wise Optimal Power Flow Formulation}

This section details the development of the line-wise optimal power flow (LWOPF) based on a set of line-wise power balance equations.

The typical OPF can be expressed as in (5.1) - (5.3)

$$
\min f o(\boldsymbol{W})
$$

subject to:

$$
\begin{array}{r}
f g(\boldsymbol{W})=d \\
\underline{h} \leq f h(\boldsymbol{W}) \leq \bar{h}
\end{array}
$$

where $\boldsymbol{W}$ is the problem vector. The function $f o(\boldsymbol{W})$ is the objective function in (5.1). The functions $f g(\boldsymbol{W})$ and $f h(\boldsymbol{W})$ are the set of functions for equality and inequality constraints respectively. In (5.2), $d$ is the target vector. In (5.3), $\underline{h}$ and $\bar{h}$ are the lower and upper limits on the inequality constraint. 


\subsubsection{Objective function}

In this formulation $\boldsymbol{W}=[U, \delta, P F, \mathrm{PS}, \mathrm{QF}, \mathrm{QS}, \mathrm{PG}, \mathrm{QG}]^{T}$, and comprises control and dependent vectors of $\boldsymbol{W C}=[\mathrm{U}, \mathrm{PG}]^{T}$ and $\boldsymbol{W D}=[\delta, P F, \mathrm{PS}, \mathrm{QF}, \mathrm{QS}, \mathrm{QG}]^{T}$ respectively.

Based on the available data for the considered tested systems, the study uses the second-order generation cost function as follows. The proposed method can be easily adapted to other nonlinear continuously differential objective functions as well.

$$
f o(\boldsymbol{W})=\sum_{g=1}^{N G} c 1_{g}+c 2_{g} \cdot P G_{g}+c 3_{g} \cdot P G_{g}{ }^{2}
$$

\subsubsection{Equality Constraints}

As reported in section 2.2, the following set of equations comprise the line-wise power balance equations, (5.5) - (5.10), and can model the entire power system. This set of equations will be used in the proposed OPF formulation to model power balance equality constraints.

Considering a pi-model of a transmission line as shown in Figure 5.1, the square of sending and receiving end voltages may be related as below in (5.5) and (5.6). A detailed derivation is provided in section 2.2 .

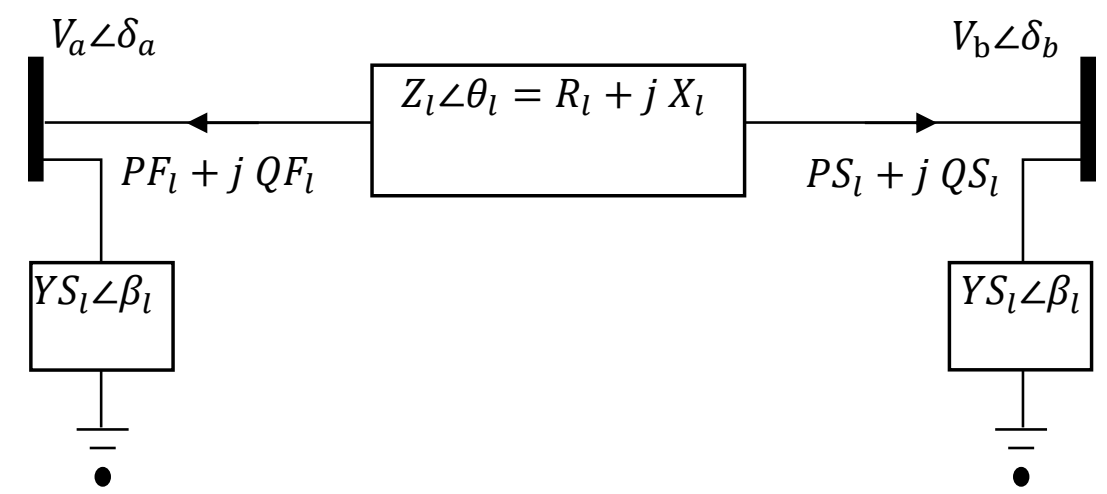

Figure 5.1 Series impedance element of the pi-model of the lth transmission branch connecting buses a and $b$.

$$
F F(\boldsymbol{W})=U_{a}{ }^{2}+2 U_{a} \cdot\left(P F_{l} \cdot R_{l}+Q F_{l} \cdot X_{l}-\frac{U_{b}}{2}\right)+S F_{l}{ }^{2} \cdot Z_{l}{ }^{2}=0 \quad \forall l=1 \text { to } N T
$$




$$
F S(\boldsymbol{W})=U_{b}{ }^{2}+2 U_{b}\left(P S_{l} \cdot R_{l}+Q S_{l} \cdot X_{l}-\frac{U_{a}}{2}\right)+S S_{l}{ }^{2} \cdot Z_{l}{ }^{2}=0 \quad \forall l=1 \text { to } N T
$$

Considering the phase angles of the sending and receiving end voltages, the relationships in (5.7) and (5.8) may be written:

$$
\begin{array}{ll}
F A(\boldsymbol{W})=\left(P F_{l} \cdot R_{l}+Q F_{l} \cdot X_{l}+U_{a}\right) \cdot \tan \left(\delta_{b}-\delta_{a}\right)-P F_{l} \cdot X_{l}+Q F_{l} \cdot R_{l}=0 & \forall l=1 \text { to } N T \\
F B(\boldsymbol{W})=\left(P S_{l} \cdot R_{l}+Q S_{l} \cdot X_{l}+U_{b}\right) \cdot \tan \left(\delta_{a}-\delta_{b}\right)-P S_{l} \cdot X_{l}+Q S_{l} \cdot R_{l}=0 & \forall l=1 \text { to } N T
\end{array}
$$

Further, the bus-wise power balance equations can be written in a matrix form as below:

$$
\begin{gathered}
F P(\boldsymbol{W})=P G+[M]\left[\begin{array}{l}
P F \\
P S
\end{array}\right]-U \cdot G S=P D \\
F Q(\boldsymbol{W})=Q G+[M]\left[\begin{array}{l}
Q F \\
Q S
\end{array}\right]+U \cdot B S=Q D
\end{gathered}
$$

where the shunt elements of lines in the pi-model and shunt loads are included in the GS and BS terms. Further, the constant loads are modelled in $P D$ and $Q D$ in (5.9) and (5.10) respectively. The set of equations (4.5) - (4.10), which model the entire power system, are considered as the set of equality constraints in the proposed LWOPF.

\subsubsection{Inequality Constraints}

In addition, the limits on the control variables are as follows.

Limits on Bus Voltages and Generators outputs are as below:

$$
\underline{U_{i}} \leq U_{i} \leq \overline{U_{i}} \quad \forall i \in N B
$$




$$
\begin{aligned}
& \underline{P G_{g}} \leq P G_{g} \leq \overline{P G_{g}} \quad \forall g \in N G \\
& \underline{Q G_{g}} \leq Q G_{g} \leq \overline{Q G_{g}} \quad \forall g \in N G
\end{aligned}
$$

Limits on MVA line power flows are as below:

$$
\begin{gathered}
P F_{l}^{2}+Q F_{l}^{2} \leq\left(\overline{S L_{l}}\right)^{2} \quad \forall l \in N T \\
P S_{l}^{2}+Q S_{l}{ }^{2} \leq\left(\overline{S L_{l}}\right)^{2} \quad \forall l \in N T
\end{gathered}
$$

The optimization problem stated in (5.4) - (5.15) is in terms of the problem vector that includes the square of voltage at each bus, the voltage angle at each bus, the active sending and receiving end powers for each branch, the reactive sending and receiving end powers for each branch, in addition to the active and reactive power generated at each generator bus.

Succinctly, the formulation (5.4) - (5.15) can be stated below.

Using (5.4), the objective function

$$
f o(\boldsymbol{W})=\sum_{g=1}^{N G} c 1_{g}+c 2_{g} \cdot P G_{g}+c 3_{g} \cdot P G_{g}{ }^{2}
$$

subject to:

the equality constraints in (5.5) - (5.10) are stated as:

$$
f g(\boldsymbol{W})=d
$$

the inequalities in (5.11) - (5.15) are stated as:

$$
\underline{h} \leq f h(\boldsymbol{W}) \leq \bar{h}
$$

In (16), the $d=\left[\begin{array}{llllll}0 & 0 & 0 & 0 & P D & Q D\end{array}\right]^{T}$. 


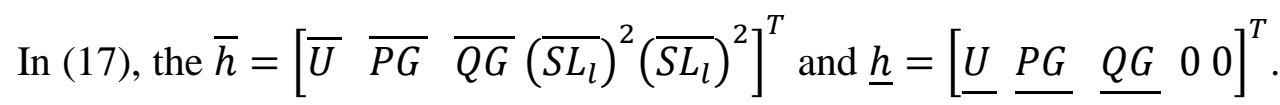

The LWOPF formulation could be summarized as the typical quadratic objective generation cost of (5.4), subjects to the sets of constraints of (5.16) and (5.17). The proposed LWOPF formulation is a nonlinear optimization problem.

\subsection{The Maximum loadability Factor (MLF) of branches}

This section includes the derivation of permissible maximum loadability factor of branches before the occurrence of the voltage collapse (VC) then discusses its effect on the solution of LWOPF.

Based on (5.6), its solution as a quadratic equation of $U_{b}$ will be as below in (5.18).

$$
\begin{gathered}
U_{b}^{1,2}=-\left(P S_{l} \cdot R_{l}+Q S_{l} \cdot X_{l}-U_{a} / 2\right) \pm \sqrt{\left(P S_{l} \cdot R_{l}+Q S_{l} \cdot X_{l}-U_{a} / 2\right)^{2}-\left(P S_{l}^{2}+Q S_{l}^{2}\right) \cdot Z_{l}^{2}} \\
=\beta_{1} \pm \beta_{2}
\end{gathered}
$$

where

$$
\begin{gathered}
\beta_{1}=-\left(P S_{l} \cdot R_{l}+Q S_{l} \cdot X_{l}-U_{a} / 2\right) \\
\beta_{2}=\sqrt{\left(P S_{l} \cdot R_{l}+Q S_{l} \cdot X_{l}-U_{a} / 2\right)^{2}-\left(P S_{l}{ }^{2}+Q S_{l}{ }^{2}\right) \cdot Z_{l}{ }^{2}}
\end{gathered}
$$

The solution exists mathematically if the square of the term $\beta_{2}$ in (5.19b) is greater than or equal to zero as in (5.20).

$$
\left(P S_{l} \cdot R_{l}+Q S_{l} \cdot X_{l}-U_{a} / 2\right)^{2}-\left(P S_{l}^{2}+Q S_{l}^{2}\right) \cdot Z_{l}^{2} \geq 0
$$

To determine the permissible $M L F$ of each branch in the system, the existing term of power $\left(P S_{l}+\right.$ $\left.j \cdot \mathrm{QS}_{l}\right)$ in (5.20) can be replaced by $M L F S_{l} \cdot\left(P S_{l}+j \cdot \mathrm{QS}_{l}\right)$ as follows in (5.21).

$$
\left(M L F S_{l} \cdot P S_{l} \cdot R_{l}+M L F S_{l} \cdot Q S_{l} \cdot X_{l}-U_{a} / 2\right)^{2}-M L F S_{l}{ }^{2} \cdot\left(P S_{l}{ }^{2}+Q S_{l}^{2}\right) \cdot Z_{l}^{2}=0
$$


By solving (5.21) as a quadratic equation of $M L F$, the maximum loadability factor of branches at receiving ends $(M L F S)$ can be obtained as follows.

$$
M L F S_{l}=\frac{U_{a} \cdot\left(P S_{l} \cdot R_{l}+Q S_{l} \cdot X_{l}-\left(P S_{l}^{2}+Q S_{l}^{2}\right) \cdot Z_{l}\right)}{2 \cdot\left[\left(P S_{l} \cdot R_{l}+Q S_{l} \cdot X_{l}\right)^{2}-\left(P S_{l}^{2}+Q S_{l}^{2}\right) \cdot Z_{l}^{2}\right]} \quad \forall l=1 \text { to } N T
$$

Similarly, for the other side, the maximum loadability factor of branches at sending ends $(M L F F)$ can be obtained as follows. Based on (5.5), its solution as a quadratic equation of $U_{b}$ will be as below in (5.23).

$$
\begin{gathered}
U_{a}^{1,2}=-\left(P F_{l} \cdot R_{l}+Q F_{l} \cdot X_{l}-U_{b} / 2\right) \pm \sqrt{\left(P F_{l} \cdot R_{l}+Q F_{l} \cdot X_{l}-U_{b} / 2\right)^{2}-\left(P F_{l}^{2}+Q F_{l}^{2}\right) \cdot Z_{l}^{2}} \\
=\beta_{3} \pm \beta_{4}
\end{gathered}
$$

where

$$
\begin{gathered}
\beta_{3}=-\left(P F_{l} \cdot R_{l}+Q F_{l} \cdot X_{l}-U_{b} / 2\right) \\
\beta_{4}=\sqrt{\left(P F_{l} \cdot R_{l}+Q F_{l} \cdot X_{l}-U_{b} / 2\right)^{2}-\left(P F_{l}^{2}+Q F_{l}^{2}\right) \cdot Z_{l}^{2}}
\end{gathered}
$$

The solution exists mathematically if the square of the term $\beta_{4}$ in (5.24b) is greater than or equal to zero as in $(5.25)$.

$$
\left(P F_{l} \cdot R_{l}+Q F_{l} \cdot X_{l}-U_{b} / 2\right)^{2}-\left(P F_{l}^{2}+Q F_{l}^{2}\right) \cdot Z_{l}^{2} \geq 0
$$

To find out the permissible $M L F$ of each branch in the system, the existing term of power $\left(P F_{l}+j \cdot Q F_{l}\right)$ can be replaced by $M L F F_{l} \cdot\left(P F_{l}+j \cdot Q F_{l}\right)$ in (5.26).

$$
\begin{gathered}
\left(M L F F_{l} \cdot P F_{l} \cdot R_{l}+M L F F_{l} \cdot Q F_{l} \cdot X_{l}-U_{b} / 2\right)^{2}-M L F F_{l}^{2} \cdot\left(P F_{l}^{2}+Q F_{l}^{2}\right) \cdot Z_{l}^{2}=0 \\
M L F F_{l}=\frac{U_{b} \cdot\left(P F_{l} \cdot R_{l}+Q F_{l} \cdot X_{l}-S F_{l} \cdot Z_{l}\right)}{2 \cdot\left[\left(P F_{l} \cdot R_{l}+Q F_{l} \cdot X_{l}\right)^{2}-S F_{l}^{2} \cdot Z_{l}^{2}\right]} \quad \forall l=1 \text { to } N T
\end{gathered}
$$

For each branch, there are two values for the maximum loadability factor which can be obtained from (5.22) and (5.27) at both sides of the branch. To ensure a secured operation, the maximum values of the two MLFs should not exceed the rated thermal limit of each branch. 
Based on the two sets of equations (5.22) and (5.27), the set of voltage security constraints can be written as follows.

$$
\operatorname{maximum}\left(M L F F_{l}, M L F S_{l}\right) \leq \tau \quad \forall l=1 \text { to } N T
$$

where $\tau$ is a rated loadability value of branches and it is obtained from power flow calculation at base loading case [43].

The proposed voltage stability constrained LWOPF can be collated into a generic formulation as the typical objective function of (5.4). Subject to the set of constraints in (5.16) and (5.17). In addition to, the set of constraints of (5.28).

From (5.4):

$$
\min f o(\boldsymbol{W})=C^{T} \cdot \boldsymbol{W}
$$

Subject to: from (5.16) - (5.17) and (5.28):

$$
\underline{b} \leq A \cdot \boldsymbol{W} \leq \bar{b}
$$

\subsection{Results of System Studies ( VSCLWOPF)}

The proposed VSCLWOPF is implemented in the Matlab® R2016a environment and solved using the FMINCON solver. All tests are done on a 64-bit i7 Intel Core laptop (2.6 GHz, 16 GB of RAM) with the Windows 10 operating system. Sparse matrix implementations are considered where possible.

The maximum loadability factor constraints of (5.28) are considered to ensure a secured operation of power systems in terms of voltage stability. Then the formulation becomes a voltage stability constrained LWOPF as stated in (5.4), (5.16), (5.17), and (5.28). The proposed VSCLWOPF is tested on 6-bus, 14-bus, 57-bus and 118-bus IEEE test systems and a 582-bus real power system data.

To illustrate the method, the proposed VSCLWOPF is tested with the 2-bus sample system shown in Figure 5.2. This simple two-bus system includes two generators G1, G2, and a demand load of PD connected to bus 2 . The load demand is less than the generated capacity of each generator. 


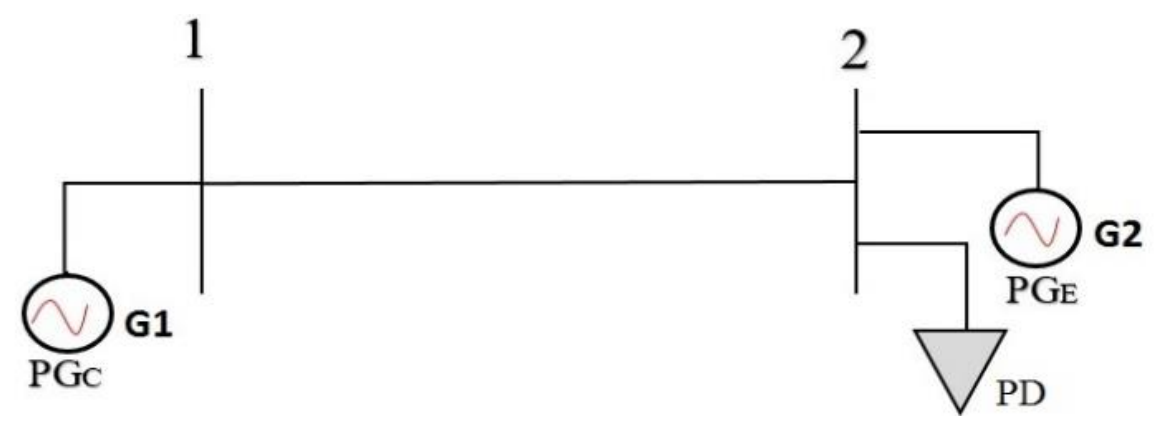

Figure 5.2 Simple two bus system.

The power $(P G C)$ of generator $G 1$ at the first bus is cheaper than the generated power $(P G E)$ of generator $G 2$ at the second bus. By applying the proposed LWOPF, it can be noted that the load demand (PD) will be completely supplied by G1 which means cheaper generation cost. To demonstrate the effects of MLF on the operation of the power systems the proposed VSCLWOPF is applied to the same simple system as follows.

Fig. 5.3. shows the effect of increasing the loadability factor (LF) on: (1) the total cost of generation of the system, and (2) the active generated power of each generator. The LF represents additional powers that can be transported through the transmission system before voltage collapse. With increasing LF, power flowing through the transmission lines that are connected to busses that are susceptible to voltage collapse will reduce, thus making those busses less susceptible. 


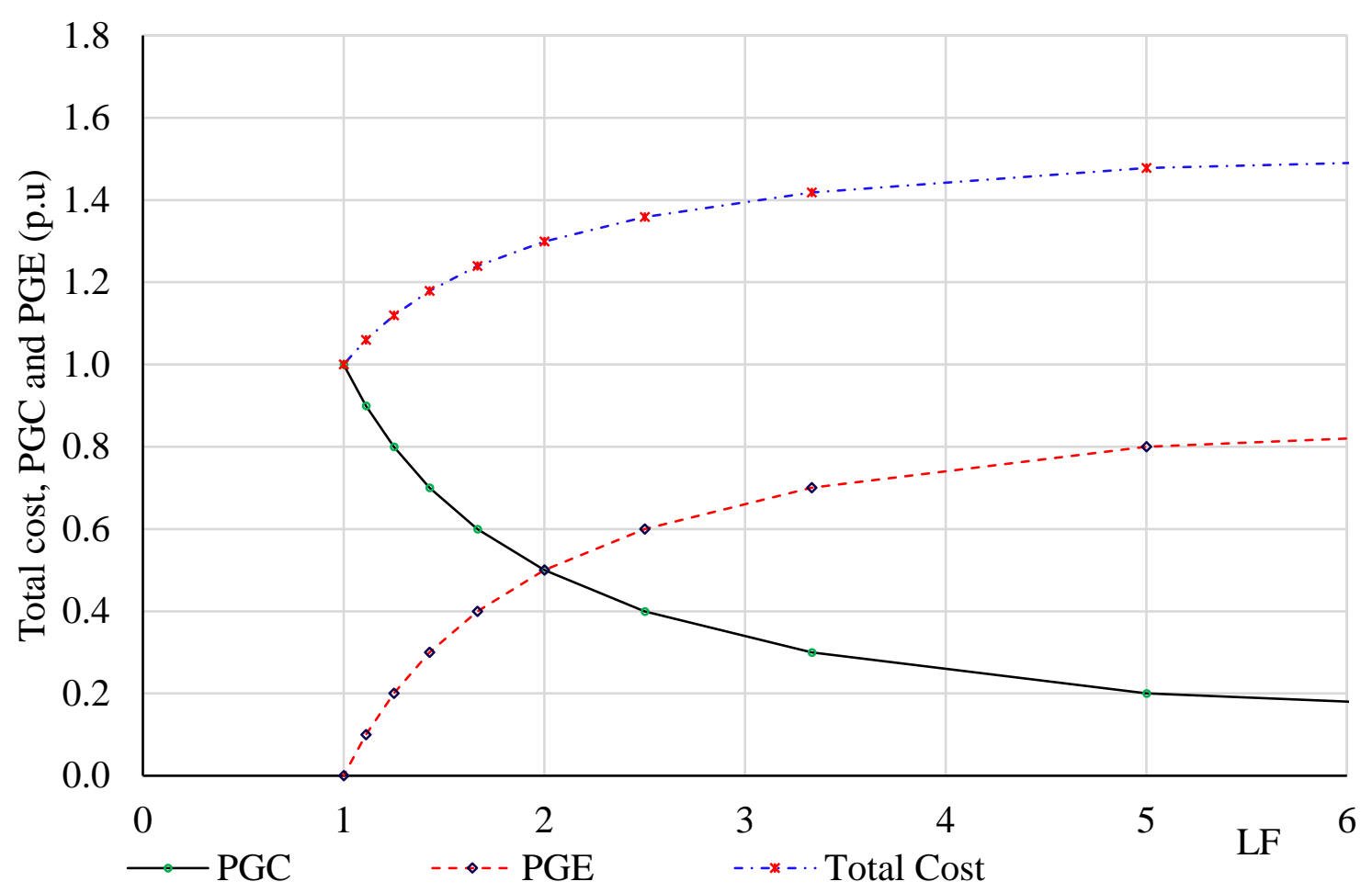

Figure 5.3 Total generation cost, PGC, and PGE values with increasing of MLF.

Accordingly, initially, the system delivers all of its load of active power from the cheaper generator "G1" at unity LF for the transmission lines. Gradually, with increasing of LF of the branch between bus 1 and bus 2, an increasing amount of power is reserved in the branch, and as a result the generated power of G1 (PGC) will be decreased, while the active generated power of G2 (PGE) will be increased to offset the reduction of PGC and maintain power balance between the total generation and total demand of the system. As a result, the cost of generation will keep growing as the LF increases.

The total cost ratio between the objective cost function before and after considering the voltage stability constraints is summarized in Fig. 5.4.

To demonstrate the effects of considering the voltage stability constraints on the solution performance, Fig. 5.4. Shows the change of objective cost function before and after inclusion of MLF constraints in the OPF for 6-bus, 14-bus, 57-bus and 118-bus benchmark systems and a real 582-bus system. Fig. 5.4 presents the percentage reduction in the generation cost, expressed in terms of the cost determined by the Voltage Stability Constrained BWOPF, required for each system to ensure the same safe operation with respect to voltage stability. This shows that by using the line-wise power flow equations in voltage stability constrained OPF, the solution domain is better (less complex and less nonlinear). This results in a lower cost solution, 
for the same voltage stability, in comparison with the same voltage stability constrained OPF with BWPF equations.

Increasing of the objective cost function in $(\%)$

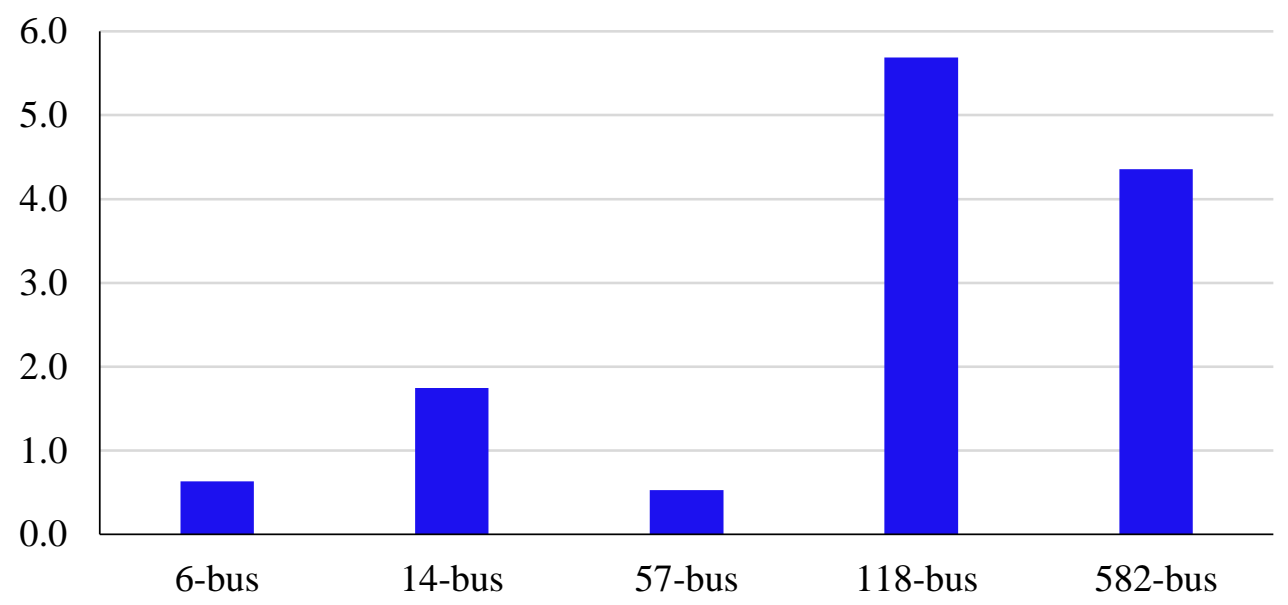

Figure 5.4 Percentage change of the objective cost function after and before considering of MLF constraints in the LWOPF for several power systems.

\subsection{Summary}

In this chapter, the Line-wise OPF formulation is augmented, incorporating the voltage stability constraints to create a voltage stability constrained LWOPF (VSCLWOPF) method. It shows that while ensuring safe operation for power systems with better voltage stability, generation cost is reduced in comparison with the bus-wise power flow based voltage stability constrained OPF formulation. The better solution space, stemming from the formulation based upon square of voltage magnitudes, yields a lower costing optimal solution for small and large sized power systems. The main contributions of this chapter can be summarized as follows:

(1) It presents a voltage collapse indicator for branches in terms of branch maximum loadability factors (MLF) which can be used for voltage stability assessment.

(2) It presents a novel voltage stability constrained line-wise optimal power flow (VSCLWOPF) with MLF as a voltage stability constraint. 
(3) The proposed formulation VSCLWOPF is tested on several power systems: 6-bus, 14-bus, 57-bus and 118-bus benchmark systems and a real 582-bus system. The results of VSCLWOPF provide a lower cost solution in less time while ensuring the same voltage stability. 


\section{Chapter Six}

\section{Chapter 6: SucCessive Linear OPTIMization - Voltage STABility INCORPORATION With Line-WISE OPTIMAL POWER FLOW}

Ensuring the voltage stability of power systems is one of the main goals of operators and researchers. The incorporation of voltage stability with optimal power flow (OPF) has received a great deal of attention in the past few years, as in Chapter 5. In this chapter, two novel linear models of voltage stability incorporation with line-wise optimal power flow are proposed based on the set of line-wise power balance equations and its line voltage collapse index. The difference between the two models are based on the way of using the VCI. In the first model, VCI are employed as a set of voltage stability constraints while the second model is based on using VCI as an objective function. The two models are solved using successive linear programming (SLP) technique, which is in contrast to Chapter 5 where nonlinear optimization technique was used. The study results on several IEEE benchmark systems demonstrates the effectiveness of the proposed two models in getting a higher voltage stable system at lower cost.

\subsection{Introduction}

Profiting from the new line-wise power flow formulation and its line voltage collapse index (VCI) reported in chapter two, a voltage stability constrained optimal power flow (VSCOPF) method is developed in this chapter. A voltage stability constrained line-wise OPF (VSCLWOPF) formulation is proposed that employs a set of line-wise power balance equations to model the entire system.

As well as the VCIs are considered as a set of voltage stability constraint. Two models for the proposed linear voltage stability constrained LWOPF are presented. Both models are dependent on the linearized voltage collapse indices to incorporate the voltage stability in the OPF study. The main difference between the two models is the way of using the VCIs which could be either an objective function or a set of constraints. 
In this chapter, the two proposed linear voltage stability incorporation with LWOPF method are implemented and solved by SLP. The two models are tested and compared on 6-, 14-, 30- and 118-bus IEEE systems.

\subsection{Nonlinear Line-wise Optimal Power Flow Formulation and the Line Voltage Collapse Index}

In this section, the main line-wise set of power balance are used to model the entire power system. As well, the two sets of voltage collapse indices are employed as an indication factor for the proximity to voltage collapse (VC). The two optimization problems are stated in terms of the problem vector $\boldsymbol{W}=[U, \delta, P F, \mathrm{PS}, \mathrm{QF}, \mathrm{QS}, \mathrm{PG}, \mathrm{QG}]^{T}$, and comprises control and dependent vectors of $\boldsymbol{W} \boldsymbol{C}=[\mathrm{U}, \mathrm{PG}]^{T}$ and $\boldsymbol{W D}=[\delta, P F, \mathrm{PS}, \mathrm{QF}, \mathrm{QS}, \mathrm{QG}]^{T}$ respectively.

\subsubsection{Line-wise power flow formulation (LWPF) and Related Constraints}

As reported in chapter two, the following set of equations comprise the polar line-wise power balance equations, (6.1) to (6.6), and can model the entire power system. This set of equations will be used in the proposed OPF formulation to model power balance equality constraints.

Considering a pi-model of a transmission line as shown in Figure 6.1, the square of sending and receiving end voltages may be related as below in (6.1) and (6.2). A detailed derivation is provided in section 2.2 .

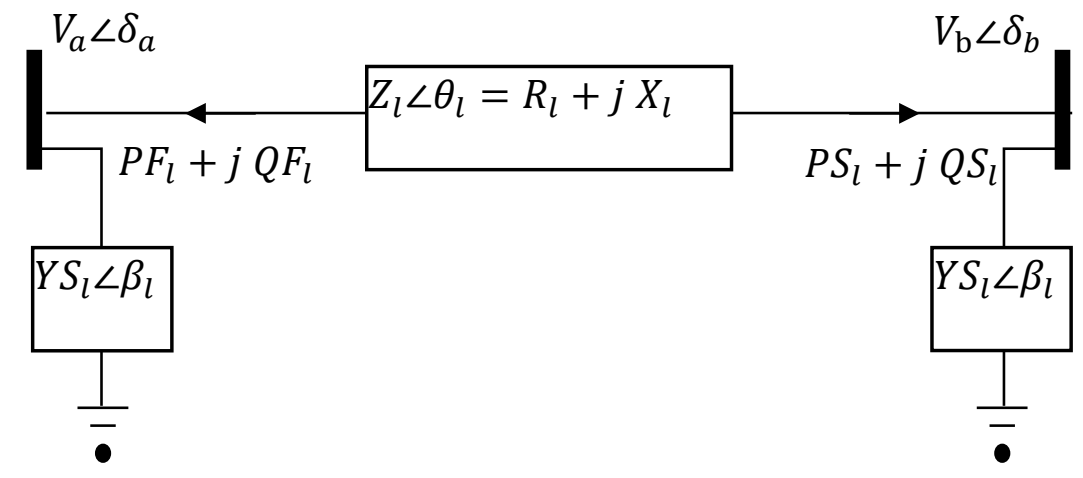

Figure 6.1 Series impedance element of the pi-model of the lth transmission branch connecting buses a and $b$. 


$$
\begin{array}{ll}
F F(\boldsymbol{W})=U_{a}{ }^{2}+2 U_{a} \cdot\left(P F_{l} \cdot R_{l}+Q F_{l} \cdot X_{l}-\frac{U_{b}}{2}\right)+S F_{l}{ }^{2} \cdot Z_{l}{ }^{2}=0 & \forall l=1 \text { to } N T \\
F S(\boldsymbol{W})=U_{b}{ }^{2}+2 U_{b}\left(P S_{l} \cdot R_{l}+Q S_{l} \cdot X_{l}-\frac{U_{a}}{2}\right)+S S_{l}{ }^{2} \cdot{Z_{l}}^{2}=0 & \forall l=1 \text { to } N T
\end{array}
$$

Considering the phase angles of the sending and receiving end voltages, the relationships may be written as follows in (6.3) and (6.4):

$$
\begin{array}{ll}
F A(\boldsymbol{W})=\left(P F_{l} \cdot R_{l}+Q F_{l} \cdot X_{l}+U_{a}\right) \cdot \tan \left(\delta_{b}-\delta_{a}\right)-P F_{l} \cdot X_{l}+Q F_{l} \cdot R_{l}=0 & \forall l=1 \text { to } N T \\
F B(\boldsymbol{W})=\left(P S_{l} \cdot R_{l}+Q S_{l} \cdot X_{l}+U_{b}\right) \cdot \tan \left(\delta_{a}-\delta_{b}\right)-P S_{l} \cdot X_{l}+Q S_{l} \cdot R_{l}=0 & \forall l=1 \text { to } N T
\end{array}
$$

Further, the bus-wise power balance equations can be written in a matrix form as below:

$$
\begin{aligned}
& F P(\boldsymbol{W})=P G+[M]\left[\begin{array}{l}
P F \\
P S
\end{array}\right]-U \cdot G S=P D \\
& F Q(\boldsymbol{W})=Q G+[M]\left[\begin{array}{l}
Q F \\
Q S
\end{array}\right]+U \cdot B S=Q D
\end{aligned}
$$

where the shunt elements of lines in the pi-model and shunt loads are included in the GS and BS terms. Further, the constant loads are modeled in PD and QD in (6.5) and (6.6) respectively.

\subsubsection{Inequality Constraints}

In addition, the limits on the control variables are as follows. Limits on Bus Voltages and Generators outputs are as below:

$$
\begin{gathered}
\underline{U_{i}} \leq U_{i} \leq \overline{U_{i}} \quad \forall i \in N B \\
\underline{P G_{g}} \leq P G_{g} \leq \overline{P G_{g}} \quad \forall g \in N G \\
\underline{Q G_{g}} \leq Q G_{g} \leq \overline{Q G_{g}} \quad \forall g \in N G
\end{gathered}
$$


Limits on MVA line power flows are as below:

$$
\begin{aligned}
& P F_{l}^{2}+Q F_{l}^{2} \leq\left(\overline{S L_{l}}\right)^{2} \quad \forall l \in N T \\
& P S_{l}^{2}+Q S_{l}^{2} \leq\left(\overline{S L_{l}}\right)^{2} \quad \forall l \in N T
\end{aligned}
$$

\subsubsection{Voltage Collapse Index}

As reported in section 2.5, for each branch in the power system, there are two line voltage collapse indices $V C I_{a}$ and $V C I_{b}$ in (6.12) and (6.13). On loading the power system, the two indices reduce to zero at voltage collapse (VC). In other words, the lower the value of the line voltage collapse index, the closer the line to the voltage collapse.

$$
\begin{aligned}
& V C I_{a}=2 \cdot U_{a}+2 \cdot\left(P F_{l} \cdot R_{l}+Q F_{l} \cdot X_{l}-\frac{U_{b}}{2}\right)>0 \\
& V C I_{b}=2 \cdot U_{b}+2 \cdot\left(P S_{l} \cdot R_{l}+Q S_{l} \cdot X_{l}-\frac{U_{a}}{2}\right)>0
\end{aligned}
$$

\subsubsection{Full Non-linear Formulation of Voltage stability incorporation with LWOPF- model \#1}

Based on the available data for the considered tested systems, the study uses the second-order generation cost function as follows. The proposed method can be easily adapted to other nonlinear continuously differential objective functions as well. Succinctly, the nonlinear voltage stability constrained LWOPF formulation can be stated below.

The objective,

$$
\min : f(\boldsymbol{W})=\sum_{g=1}^{N G} c 1_{g}+c 2_{g} \cdot P G_{g}+c 3_{g} \cdot P G_{g}{ }^{2}
$$

subject to:

the equality constraints in $(6.1)-(6.6)$ are stated as: 


$$
f g(\boldsymbol{W})=d
$$

the inequalities in $(6.7)-(6.11)$ are stated as:

$$
\underline{h} \leq f h(\boldsymbol{W}) \leq \bar{h}
$$

Further, the voltage stability constraints of (6.12) - (6.13) are stated as:

$$
\operatorname{Min}\left(V C I_{l}\right) \geq V C I_{\text {limit }} \quad \forall l \in N T
$$

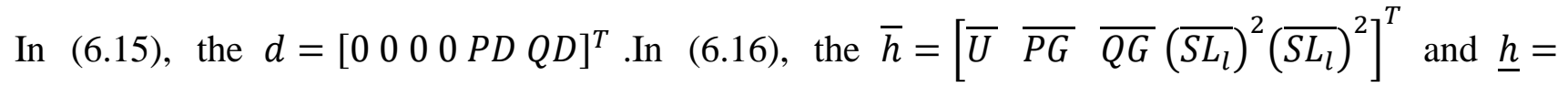
$\left[\begin{array}{llll}\underline{U} & \underline{P G} \underline{Q G} & 0 & 0\end{array}\right]^{T}$.

\subsubsection{Full Non-linear Formulation of Voltage stability incorporation with LWOPF- model \#2}

This subsection presents the second option of proposed nonlinear voltage stability incorporation with LWOPF. The objective function is to maximize the sum of all voltage collapse indices.

$$
\text { Max. } \quad \sum_{l=1}^{N T} V C I_{a_{l}}+V C I_{b_{l}}
$$

subject to same equality and inequality sets of equations mentioned in (15) and (16):

the equality constraints in $(6.1)-(6.6)$ are stated as:

$$
f g(\boldsymbol{W})=d
$$

the inequalities in (6.7) $-(6.11)$ are stated as: 


$$
\underline{h} \leq f h(\boldsymbol{W}) \leq \bar{h}
$$

\subsection{Development of Linear Models of Objective Function, Equality Constraints, Inequality Constraints and VCI indices - models 1 and 2.}

In this section, a linear incremental model is presented below. The problem vector $\Delta \boldsymbol{W}$ comprises control and dependent vectors of $\Delta \boldsymbol{W C}=[\Delta U, \Delta P G]^{T}$ and $\Delta \boldsymbol{W D}=[\Delta \delta, \Delta P F, \Delta P S, \Delta Q F, \Delta Q S, \Delta Q G]^{T}$ respectively. The linearized problem vector is:

$\Delta \boldsymbol{W}=[\Delta U, \Delta \delta, \Delta P F, \Delta P S, \Delta Q F, \Delta Q S, \Delta P G, \Delta Q G]^{T}$.

\subsubsection{Linearized Objective Function - model \#1}

The linearized objective function from (6.14) will be as follows.

$$
\text { Min. } \quad \Delta f o(\boldsymbol{W})=\left[\frac{d f o(\boldsymbol{W})}{d \boldsymbol{W}}\right] \cdot \Delta \boldsymbol{W}
$$

or

$$
\text { Min. } \Delta f o(\Delta \boldsymbol{W})=\sum_{g=1}^{N G}\left[\left(c 2_{g}+2 \cdot c 3_{g} \cdot P G_{g}\right) \cdot \Delta P G_{g}\right]
$$

\subsubsection{Linearized Objective Function - model \#2}

This objective function is to maximize the sum of all voltage collapse indices.

$$
\text { Max. } \quad \sum_{l=1}^{N T} \Delta V C I_{a_{l}}+\Delta V C I_{b_{l}}
$$

where

$$
\begin{array}{cc}
\Delta V C I_{a_{l}}=\frac{\partial V C I_{a_{l}}}{\partial U} \Delta U+\frac{\partial V C I_{a_{l}}}{\partial P F} \Delta P F+\frac{\partial V C I_{a_{l}}}{\partial Q F} \Delta Q F & \forall l \in N T \\
\Delta V C I_{b_{l}}=\frac{\partial V C I_{b_{l}}}{\partial U} \Delta U+\frac{\partial V C I_{b_{l}}}{\partial P S} \Delta P S+\frac{\partial V C I_{b_{l}}}{\partial Q S} \Delta Q S & \forall l \in N T
\end{array}
$$




\subsubsection{Linearized Set of Equality Constraints}

The linearized set of equality constraints (6.15) is as below:

$$
\Delta \mathrm{f} g(\boldsymbol{W})=\left[\begin{array}{ll}
\boldsymbol{J} & \boldsymbol{D}
\end{array}\right] \Delta \boldsymbol{W}=\Delta d
$$

where $\boldsymbol{J}$ is the Jacobian matrix of the set of line-wise power balance equations (6.15), as derived in [10], and shown below in (6.24), and $\boldsymbol{D}$ is a submatrix includes two vectors for the partial differentiation of the set (6.1) to (6.6) for to $P G$ and $Q G$ respectively as follows in (6.25).

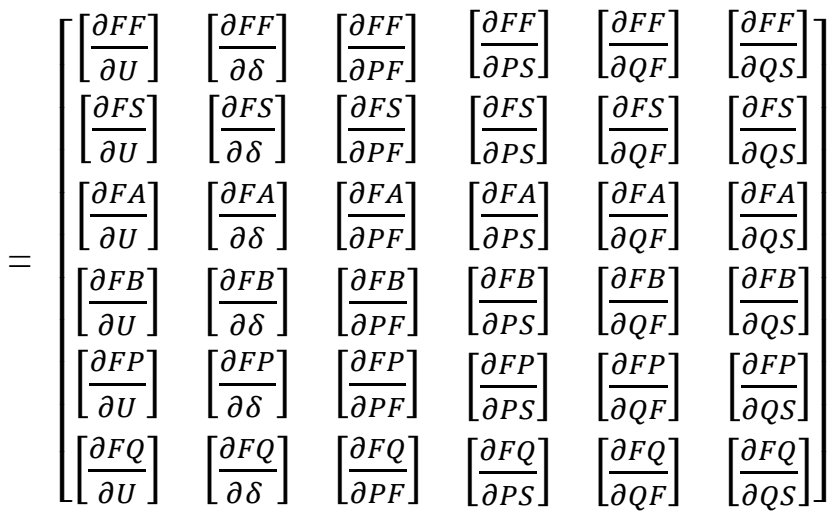

$$
\begin{aligned}
& D=\left[\begin{array}{llllll}
\frac{\partial F F}{\partial P_{G}} & \frac{\partial F S}{\partial P_{G}} & \frac{\partial F A}{\partial P_{G}} & \frac{\partial F A}{\partial P_{G}} & \frac{\partial F P}{\partial P_{G}} & \frac{\partial F Q}{\partial P_{G}} \\
\frac{\partial F F}{\partial Q_{G}} & \frac{\partial F S}{\partial Q_{G}} & \frac{\partial F B}{\partial Q_{G}} & \frac{\partial F B}{\partial Q_{G}} & \frac{\partial F P}{\partial Q_{G}} & \frac{\partial F Q}{\partial Q_{G}}
\end{array}\right]^{T}
\end{aligned}
$$

The vector $\Delta d=\left[\begin{array}{llllll}0 & 0 & 0 & 0 & 0 & 0\end{array}\right]^{T}$

\subsubsection{Linearized Set of Inequality Constraints}

Further, the set of limits on the control variables in (6.7) to (6.11) could be linearized as follows. Considering the current operating value $\boldsymbol{W}^{\circ}$ and a step size of $\alpha$ :

$$
\underline{\Delta U_{i}} \leq \Delta U_{i} \leq \overline{\Delta U_{i}} \quad \forall i \in N B
$$

where $\underline{\Delta U_{i}}=\alpha \cdot\left(\underline{U_{i}}-U_{i}^{0}\right)$ and $\overline{\Delta U_{i}}=\alpha \cdot\left(\overline{U_{i}}-U_{i}^{0}\right)$. 


$$
\underline{\Delta P G_{g}} \leq \Delta P G_{g} \leq \overline{\Delta P G_{g}} \quad \forall g \in N G
$$

where $\underline{\Delta P G_{g}}=\alpha \cdot\left(\underline{P G_{g}}-P G_{g}{ }^{0}\right)$ and $\overline{\Delta P G_{g}}=\alpha \cdot\left(\overline{P G_{g}}-P G_{g}{ }^{0}\right)$.

$$
\underline{\Delta Q G_{g}} \leq \Delta Q G_{g} \leq \overline{\Delta Q G_{g}} \quad \forall g \in N G
$$

where $\left.\underline{\Delta Q G_{g}}=\alpha \cdot \underline{Q G_{g}}-Q G_{g}{ }^{0}\right)$ and $\overline{\Delta Q G_{g}}=\alpha \cdot\left(\overline{Q G_{g}}-Q G_{g}{ }^{0}\right)$.

$$
\begin{array}{ll}
2 \cdot P F_{l} \cdot \Delta P F_{l}+2 \cdot Q F_{l} \cdot \Delta Q F_{l} \leq \overline{\Delta S L_{l}} & \forall l \in N T \\
2 \cdot P S_{l} \cdot \Delta P S_{l}+2 \cdot Q S_{l} \cdot \Delta Q S_{l} \leq \overline{\Delta S L_{l}} & \forall l \in N T
\end{array}
$$

where $\overline{\Delta S L_{l}}=\alpha \cdot\left(\overline{S L_{l}}-S L_{l}{ }^{0}\right)$.

\subsubsection{Linearized Voltage Collapse Indices for Model \#1 only}

In this section, two options for the proposed linear voltage stability constrained LWOPF are presented. Both options depend on the linearized voltage collapse indices to incorporate the voltage stability in the OPF study. The main difference between the two options is the way of using the voltage collapse indices which could be either an objective function or a set of constraints.

The two sets of linearized voltage collapse indices could be developed as follows.

$$
\begin{array}{ll}
\Delta V C I_{a_{l}}=\frac{\partial V C I_{a_{l}}}{\partial U} \Delta U+\frac{\partial V C I_{a_{l}}}{\partial P F} \Delta P F+\frac{\partial V C I_{a_{l}}}{\partial Q F} \Delta Q F & \forall l \in N T \\
\Delta V C I_{b_{l}}=\frac{\partial V C I_{b_{l}}}{\partial U} \Delta U+\frac{\partial V C I_{b_{l}}}{\partial P S} \Delta P S+\frac{\partial V C I_{b_{l}}}{\partial Q S} \Delta Q S & \forall l \in N T
\end{array}
$$




\subsection{Two proposed Linear Models of Voltage Stability incorporation with LWOPF}

In this section, the two models of linear voltage stability incorporation with LWOPF are developed.

\subsubsection{Linear Voltage Stability incorporation with LWOPF- Model \#1 (VCI as set of constraints)}

In this subsection, the first option of linear voltage stability constrained LWOPF is developed. The proposed formulation includes the normal linear LWOPF and the linearized voltage stability constraints.

The two sets of voltage stability constraints are employed as follows.

$$
\operatorname{Min}\left(\triangle V C I_{l}\right) \geq \Delta V C I_{\text {limit }}
$$

$\Delta V C I_{\text {limit }}$ : Threshold value of voltage collapse indices.

The linear voltage stability constrained LWOPF formulation can be collated into a generic formulation as below:

From (6.21b): the objective function

$$
\text { Min. } \Delta f o(\boldsymbol{W})=C^{T} \cdot \Delta \boldsymbol{W}
$$

Subject to the set of constraints from $(6.23)-(6.30)$ :

$$
\underline{b} \leq A \cdot \Delta \boldsymbol{W} \leq \bar{b}
$$

In addition to the linearized voltage stability constraints from (6.32)

$$
\operatorname{Min}\left(\Delta V C I_{l}\right) \geq \Delta V C I_{\text {limit }} \quad \forall l \in N T
$$




\subsubsection{Linear Voltage Stability incorporation with LWOPF- Model \#2 (VCI as an objective function)}

This subsection presents the second option of proposed linear voltage stability incorporation with LWOPF. The linear voltage stability incorporation with LWOPF formulation can be collated into a generic formulation as below:

From $(6.22 \mathrm{a})-(6.22 \mathrm{c})$ : the objective function

$$
\operatorname{Max} . \quad \sum_{l=1}^{N T} \Delta V C I_{a_{l}}+\Delta V C I_{b_{l}}
$$

Subject to the set of constraints from $(6.22)-(6.29)$ :

$$
\underline{b} \leq A \cdot \Delta \boldsymbol{W} \leq \bar{b}
$$

As noted before, both proposed linear voltage stability incorporation with LWOPF formulations can be solved using any linear optimization method such as Simplex, interior point algorithm, etc. In this study, the successive linear optimization is employed to solve the two proposed formulation as can be shown in the following section.

\subsection{Successive Linear Programming Algorithm}

This section describes the proposed algorithm to solve the voltage stability constrained LWOPF formulation using SLP technique as shown in Figure 6.2. The algorithm includes two main steps in each iteration: first, solving the line-wise power flow; second, linearization of the nonlinear LWOPF formulation including the voltage collapse indices around the operating point obtained from the first step, then solving the resultant linear optimization problem using a chosen LP technique. The entire procedure, the two steps, will be repeated successively till convergence based on the stopping criterion is reached. It may be useful here to note that the algorithm to solve the set of line-wise power flow, the (6.1) to (6.6) formulation, was explained before in [10] based on Newton-Raphson method. The stopping criterion of the proposed algorithm is as below in (6.39) which is similar to that of [104]. 


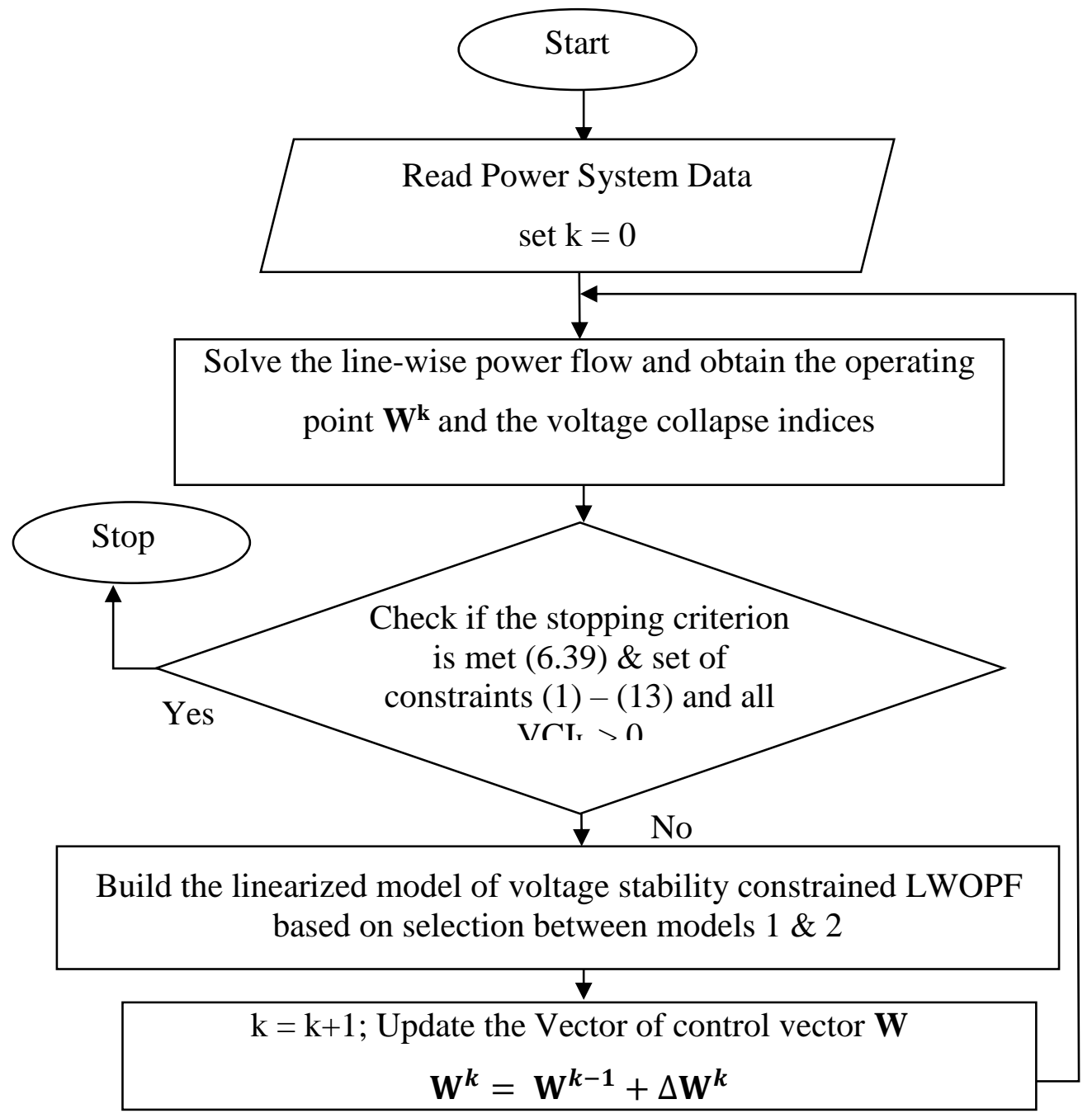

Figure 6.2 Flowchart of proposed LWOPF based on SLP.

The stopping criterion of SLP algorithm is based on satisfy the condition in (6.39) and all the set of constraints $(6.1)-(6.13)$ including that all VCI for all branches are greater than zero.

$$
\frac{\left|f o\left(\boldsymbol{W}^{k}\right)-f o\left(\boldsymbol{W}^{k-1}\right)\right|}{\left|f o\left(\boldsymbol{W}^{k}\right)\right|} \leq \gamma
$$

\subsection{Results of system studies}

In this section, test results of the two proposed linear models of voltage stability incorporation with line-wise OPF are presented and compared for several IEEE bench mark systems. The proposed algorithm 
is implemented in the Matlab® R2016a environment and solved using the Mosek 7.0 solver. All tests are done on a 64-bit i7 Intel Core laptop (2.6 GHz, $16 \mathrm{~GB}$ of RAM) with the Windows 10 operating system. Sparse matrix implementations are considered where possible. Solutions are obtained for all case studies at mismatch tolerance of $\gamma=10^{-6}$.

To demonstrate the effectiveness of the proposed two linear models for incorporation of voltage stability with the line-wise OPF, the tested systems are stressed by scaling the load buses by a loading factor (LF) greater than one or assuming a contingency case such as line outage.

\subsubsection{Stressed system studies}

In this subsection, the results of solving several IEEE systems based on the two proposed linear models of incorporation of the voltage stability with the LWOPF are compared with the solution of normal linear LWOPF to demonstrate the impacts of considering the voltage stability on the operation performance. The systems are stressed by scaling their load bused by a LF up to the loading at which collapse will occur based on results reported in chapter two section 2.9.

Figure 6.3, Figure 6.5, and Figure 6.7 show the comparison between the cost function value for the solutions of the normal LWOPF and the two proposed models of incorporating the voltage stability with LWOPF at several LF for Ward-Hale 6-bus, IEEE 14- and 118-bus systems respectively. It can be shown that incorporation the voltage stability may lead to extra cost to ensure secured operation. In Figure 6.4, Figure 6.6, and Figure 6.8, the minimum VCI at several LF are compared for the solution of normal LWOPF and the two proposed linear models at several LF for Ward-Hale 6-bus, IEEE 14- and 118-bus systems respectively. It can be noted that the second model which considers the $V C I$ as an objective function has higher minimum $V C I$ than the first model.

a) Ward-Hale 6-bus system 


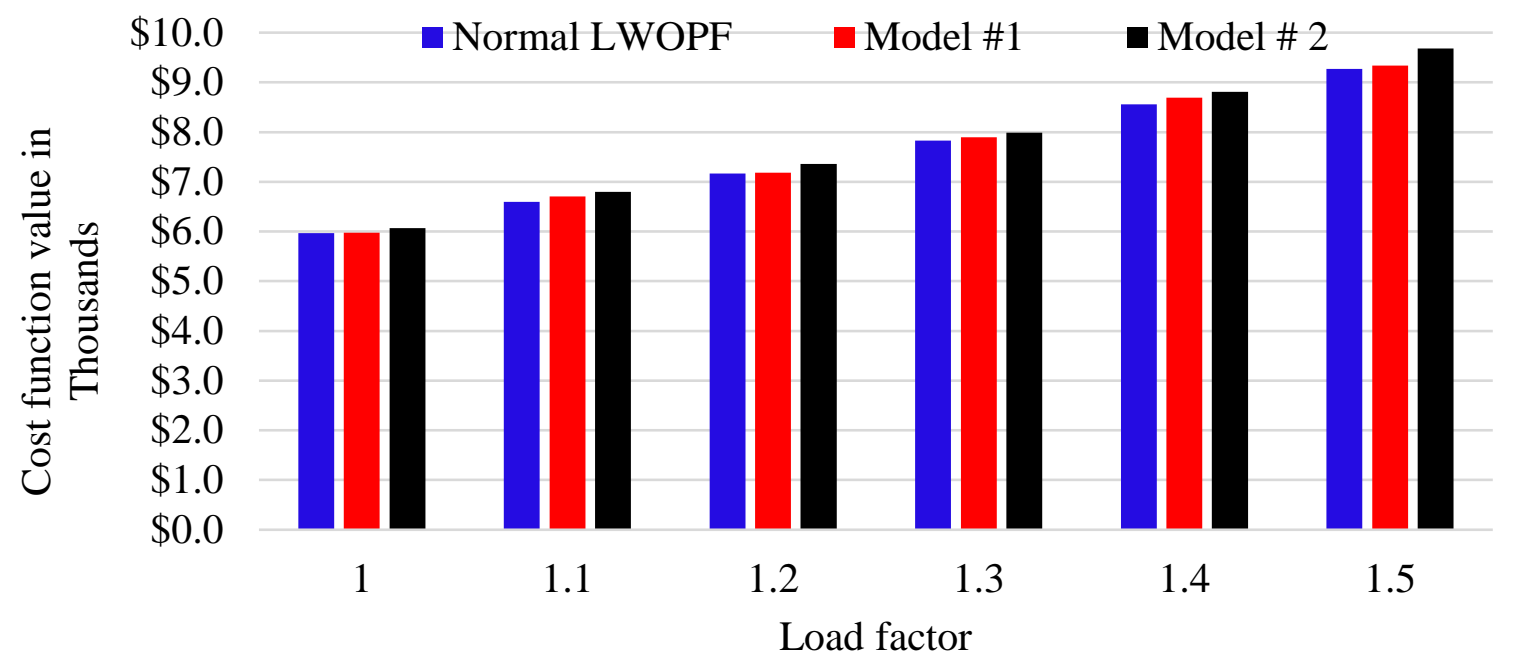

Figure 6.3 Comparison between cost function value of the two proposed models and the normal LWOPF of Ward-Hale 6-bus system at several loading factors (stressed).

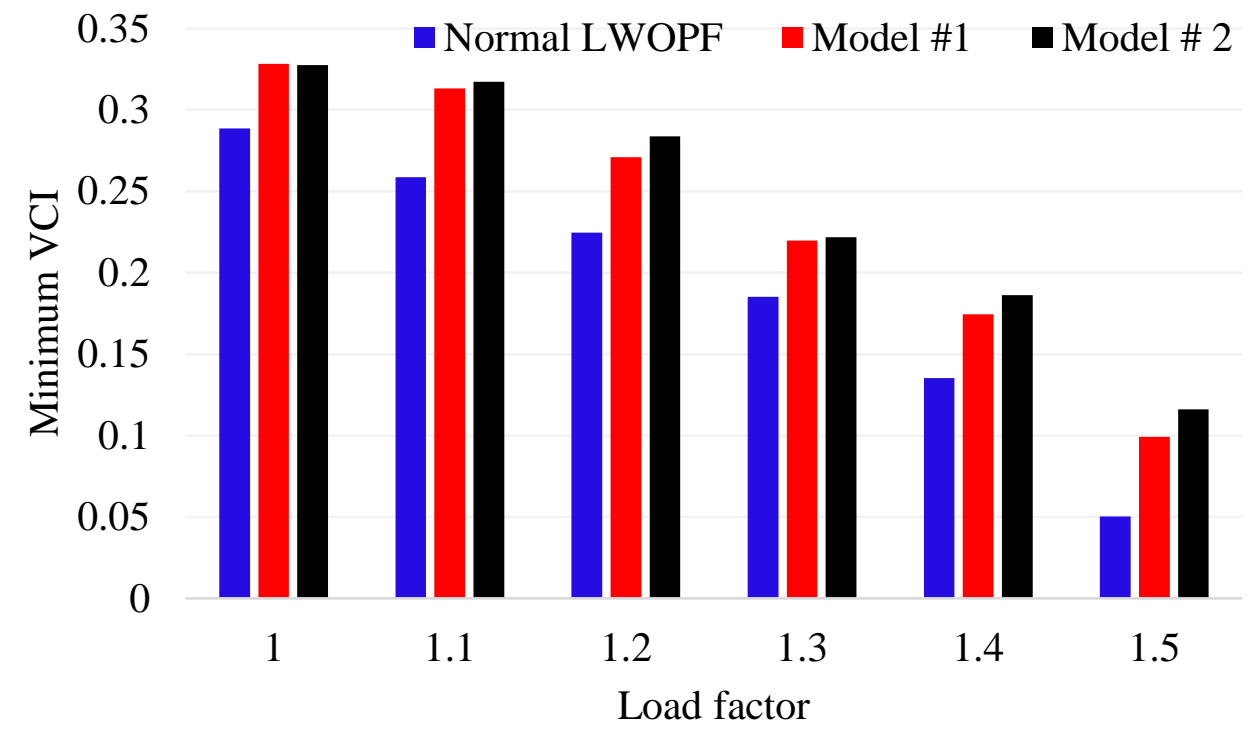

Figure 6.4 Comparison between the value of minimum VCI of the solution of the two proposed models and the normal LWOPF of Ward-Hale 6-bus system at several loading factors (stressed).

b) IEEE 14-bus system 


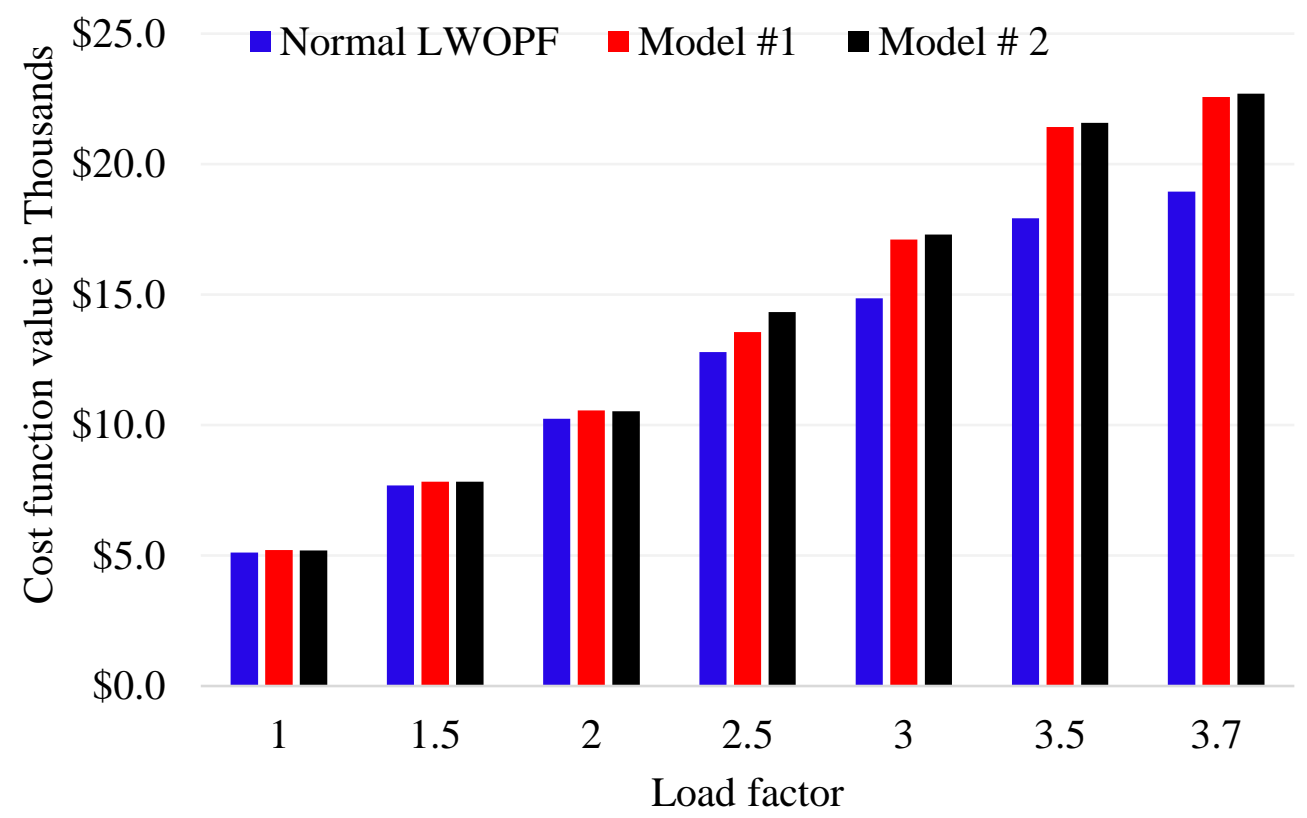

Figure 6.5 Comparison between the cost function value of the solution of the two proposed models and the normal LWOPF of 14-bus system at several loading factors (stressed).

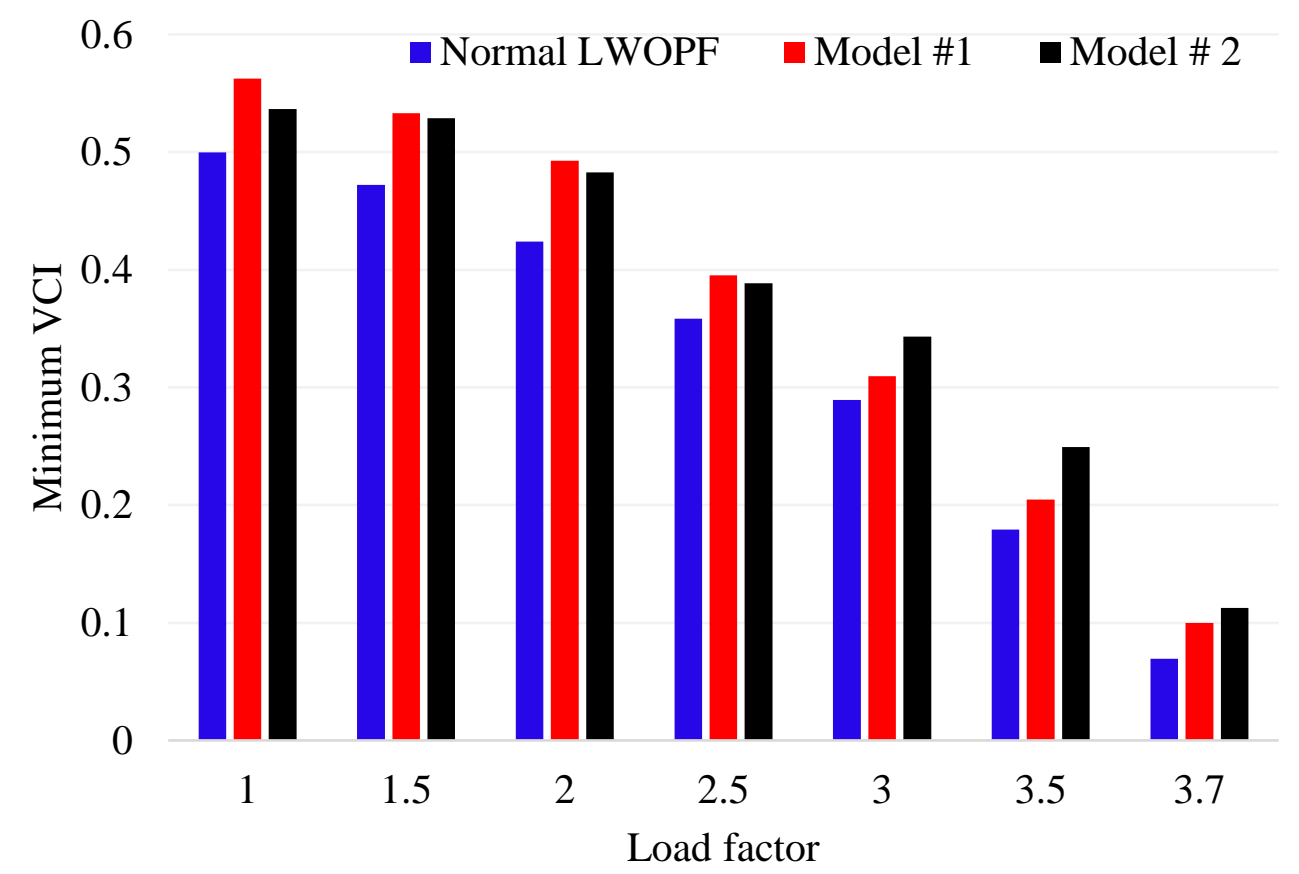

Figure 6.6 Comparison between the value of minimum VCI of the solution of the two proposed models and the normal LWOPF of 14-bus system at several loading factors (stressed).

c) IEEE 118-bus system 


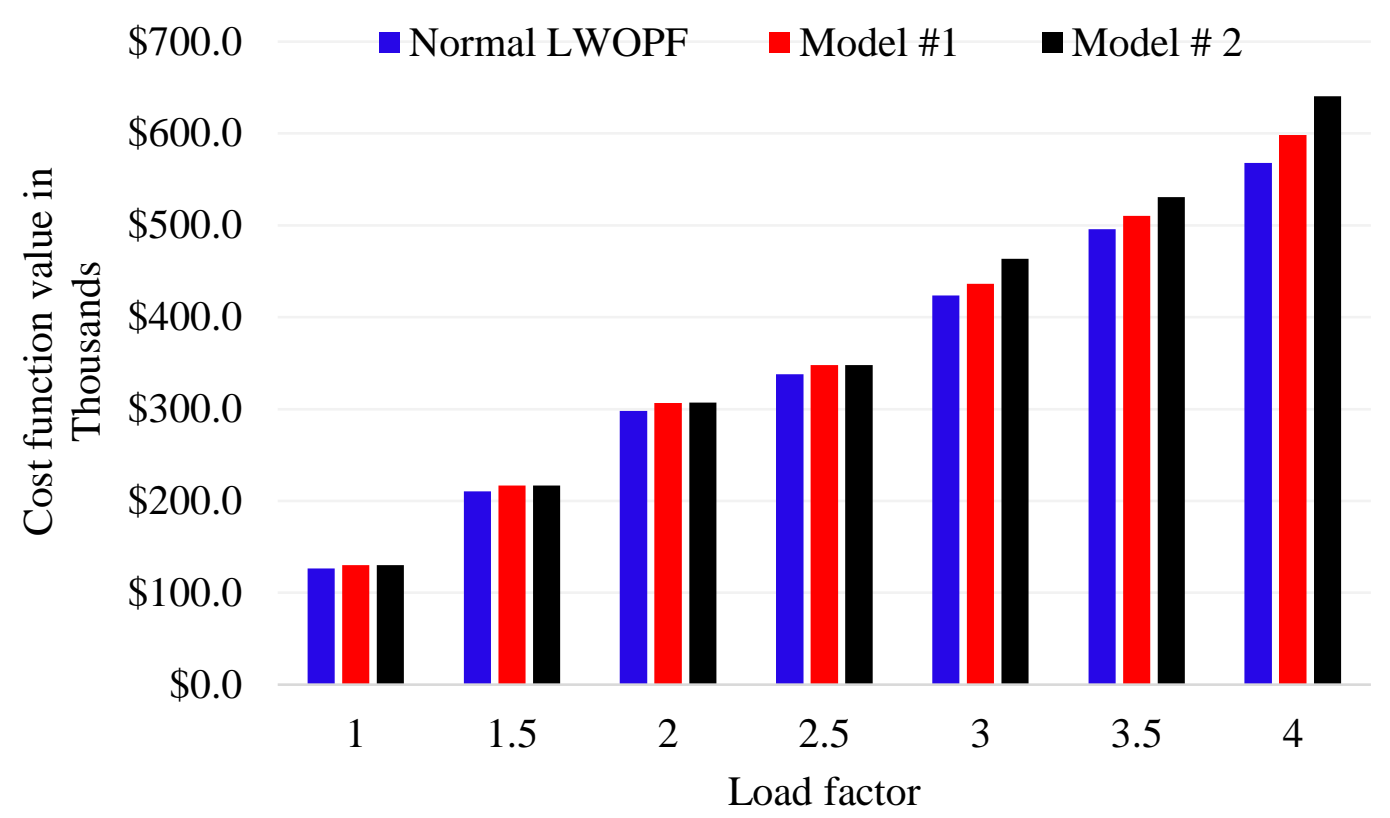

Figure 6.7 Comparison between the cost function value of the solution of the two proposed models and the normal LWOPF of 118-bus system at several loading factors (stressed).

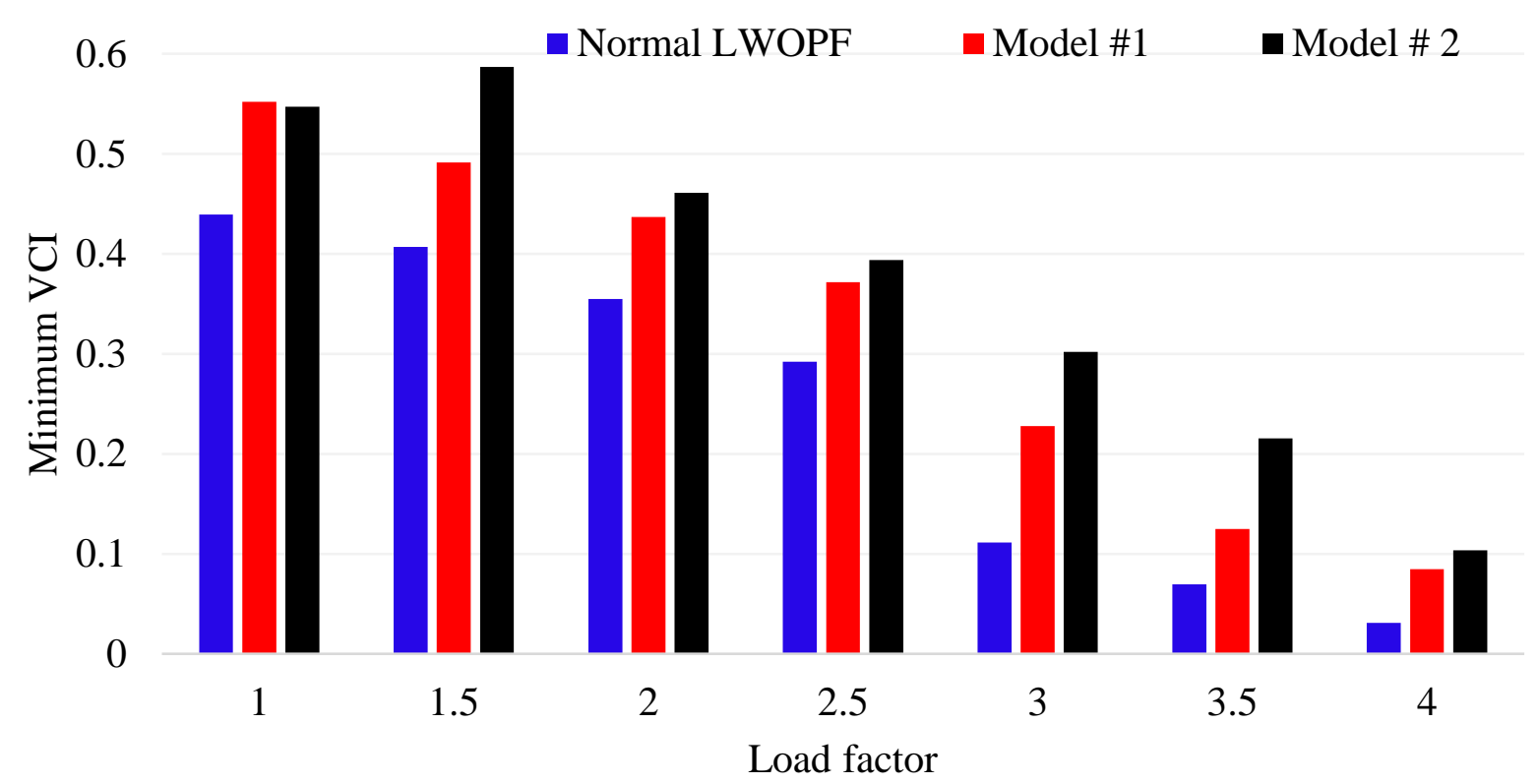

Figure 6.8 Comparison between the value of minimum VCI of the solution of the two proposed models and the normal LWOPF of 118-bus system at several loading factors (stressed). 


\subsubsection{Outage system studies}

In this subsection, the results of applying the proposed models of voltage stability incorporation with OPF to systems under contingency are presented. The contingency analysis considers an outage of one of the system branches as follows.

For Ward-Hale 6-bus system, an outage case of line (L4-6) between buses 4 and 6 is tested and comparison is reported in Table 1. The results show that applying the proposed models of voltage stability incorporation with OPF is combined with an increase in the cost function, however, the system performance is improved in terms of better voltage stability conditions, lower losses. In addition, the enhancement of the bus voltage magnitudes is shown in Figure 6.9.

Table 6.1 Comparison between the results of two proposed models and normal LWOPF for a line outage of Ward-Hale 6-bus system.

\begin{tabular}{c|c|c|c}
\hline & Normal LWOPF & Model \#1 & Model \#2 \\
\hline Cost (\$) & 5963.30 & 5976.51 & 6068.89 \\
\hline $\begin{array}{c}\text { Minimum Voltage } \\
\text { Collapse Index }\end{array}$ & 0.2208 & 0.2495 & 0.2642 \\
\hline Losses (p.u.) & 0.12 & 0.096 & 0.078 \\
\hline $\begin{array}{c}\text { Reactive Power } \\
\text { Generation (p.u.) }\end{array}$ & 0.388 & 0.368 & 0.372 \\
\hline
\end{tabular}

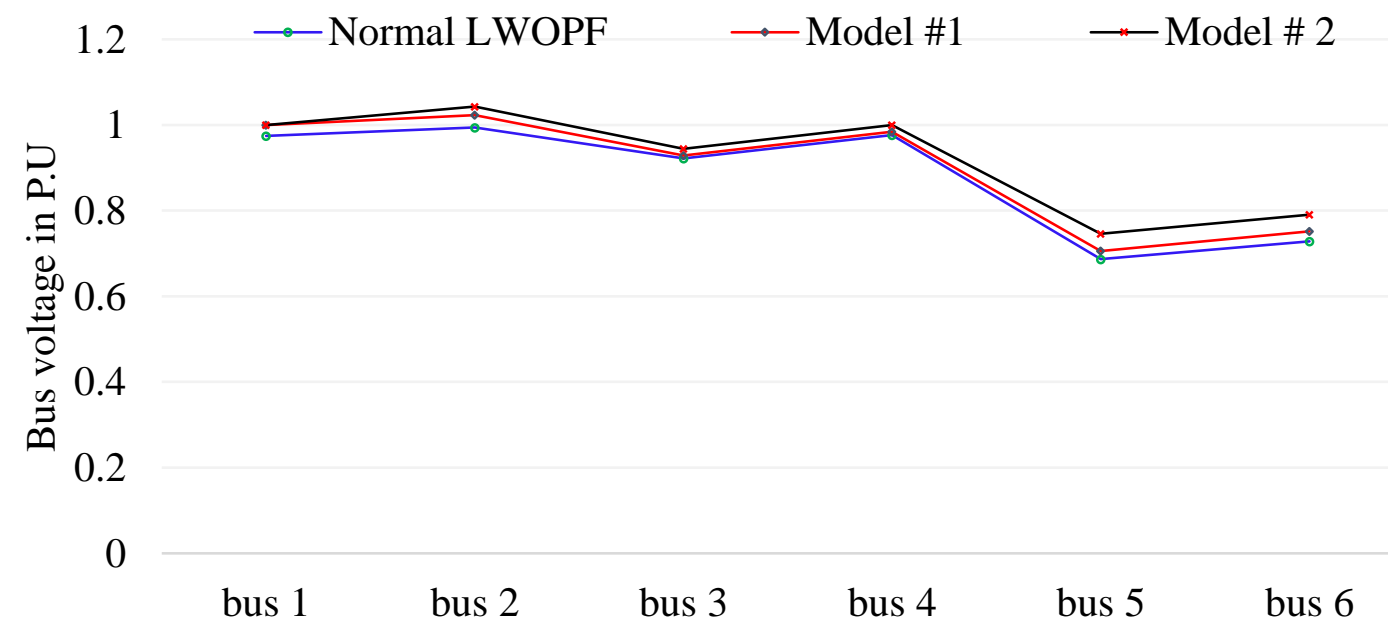

Figure 6.9 Enhancement of bus voltages of the two proposed models compared to the normal LWOPF of Wald-Hale 6-bus system with a line outage contingency. 


\subsubsection{Comparison with an existing well-known voltage stability constrained OPF (IEEE 30-bus system)}

To emphasize the quality and performance of the proposed two models, a comparison is accomplished between the results of two proposed models of incorporation the voltage stability with LWOPF and the results of an existing algorithm of voltage stability constrained OPF in [127]. Comparison study considers the IEEE 30-bus system in two conditions; first, stressed system by scaling load buses by a LF of 1.4 and second, a line outage for line (L2-5) between buses 2 and 5.

Table 6.2 and Table 6.3 present the superiority of the proposed models voltage stability incorporation with LWOPF over the one of the well-known VSCOPF which based on the voltage collapse proximity factor (VCPF). To ensure a fair comparison, the voltage collapse proximity indicator (VCPI), which presents the ratio between the receiving end active power to the maximum allowable power transfer through the branch, of [127] is calculated for the solution of each model as shown in Table 6.2 and Table 6.2

Table 6.2 Comparison between the results of two proposed models and normal LWOPF with best results of an existing VSCOPF in [127] for a stressed IEEE 30-bus system at 1.4 load factor.

\begin{tabular}{c|c|c|c|c}
\hline \multirow{2}{*}{} & \multicolumn{2}{|c|}{ Proposed models based on LWOPF } & \multirow{2}{*}{$\begin{array}{c}\text { Existing VSCOPF } \\
\text { of [127] }\end{array}$} \\
\cline { 2 - 4 } & LWOPF & Model \#1 & Model \#2 & 1403.04 \\
\hline $\begin{array}{c}\text { Cost }(\$) \\
\text { Losses } \\
(\text { MW })\end{array}$ & 1259.91 & 1294.91 & 1297.46 & 8.616 \\
\hline $\begin{array}{c}\text { Reactive } \\
\text { Power } \\
\text { Generation } \\
(\text { Mvar })\end{array}$ & 13.14 & 5.73 & 11.54 & 158.76 \\
\hline $\begin{array}{c}\text { VCPI } \\
\text { MAX }\end{array}$ & 0.367 & 154.02 & 123.25 & 0.260 \\
\hline VCPI & 4.664 & 0.258 & 0.254 & 4.166 \\
\hline
\end{tabular}


Table 6.3 Comparison between the results of two proposed models and normal LWOPF with best results of an existing VSCOPF in [127] for a IEEE 30-bus system with line outage for (L2-5).

\begin{tabular}{c|c|c|c|c}
\hline \multirow{2}{*}{} & \multicolumn{2}{|c|}{ Proposed models based on LWOPF } & \multirow{2}{*}{$\begin{array}{c}\text { Existing VSCOPF of } \\
\text { [127] }\end{array}$} \\
\cline { 2 - 5 } & LWOPF & Model\#1 & Model\#2 & 1174.91 \\
\hline $\begin{array}{c}\text { Cost }(\$) \\
(\text { MW })\end{array}$ & 833.75 & 935.56 & 928.80 & 9.518 \\
\hline $\begin{array}{c}\text { Qgen } \\
(\text { Mvar })\end{array}$ & 138.50 & 126.94 & 68.514 & 134.17 \\
\hline $\begin{array}{c}\text { VCPI } \\
\text { MAX }\end{array}$ & 0.330 & 0.222 & 0.220 & 0.222 \\
\hline VCPI & 4.391 & 3.765 & 3.762 & 3.769 \\
\hline
\end{tabular}

It can be noted form the comparisons of tables 6.2 and 6.3 that the two proposed models have better performance in terms of generation cost, losses, and reactive power generation compared to the existing algorithm of [127].

\subsection{Summary}

In this chapter, two new linear models for incorporating the voltage stability with the optimal power flow based on a set of line-wise power flow equations and line voltage collapse index are proposed. The line-wise optimal power flow (LWOPF) formulation is linearized and incorporated with the set of linearized voltage collapse indices in two ways; first, VCIs are considered as a set of constraints, second, sum of VCIs is considered as an objective function. The two proposed models are solved using successive linear programming technique (SLP). The two linear proposed models for incorporation the voltage stability with LWOPF are tested on 6-, 14-, and 118-bus IEEE systems and compared with the solution of linearized linewise OPF (LWOPF).

The results show the effectiveness of the proposed models to enhance the voltage stability for power systems even under severe contingency conditions such as line outage or stressed loading. The proposed models are compared with an existing voltage stability constrained optimal power flow methods and show a better performance in terms of minimizing the cost function and getting a better voltage stable system. 


\section{Chapter Seven}

\section{ChAPTER 7: CONCLUSION}

\subsection{General}

Power flow has a closer relationship to square of the magnitude of voltage than to magnitude of voltage itself. Hence, power balance equations developed using square of voltage magnitude yield simpler (less complex) equations, a lower number of higher order terms and a better solution space. This thesis is about exploiting this understanding to create power balance equations and optimal power flow methods based upon this new set of power balance equations. The benefits include faster power flow algorithms, faster optimal power flow algorithms, better optimal solution using OPF, and more voltage stable solutions.

The work done in this thesis can be summarized as follows; In Chapter 2, the mathematical formulation of the polar line-wise PF based on NR method and the line voltage collapse index are developed. Further, the comparison of results with existing well-known methods is presented to show the superiority of the developed polar line-wise PF. Chapter 3 presents line-wise power flow in the rectangular form. The rectangular version of line-wise PF formulation is solved based on NR technique and compared with existing methods.

In Chapter 4, benefiting from the developed polar line-wise PF of Chapter 2, a linearized incremental line-wise optimal power flow (LWOPF) is developed and solved based on successive linear programming (SLP). The results show better performance in terms of solution space and execution time compared to wellknown existing methods. In Chapter 5, a nonlinear formulation for polar line-wise optimal power flow is developed considering maximum loadability factors (MLF) for the transmission branches. The MLF is employed to create a nonlinear voltage stability constrained optimal power flow. Chapter 6 shows the development of two proposed linearized models of incorporating the voltage stability with the linearized LWOPF based on the polar line-wise PF and the VCI developed in Chapter 2. The solution algorithm for the proposed two models is based on the SLP technique and results are compared with existing work to show the superior performance. 


\subsection{Contribution and main benefits of thesis}

The main contributions and benefits of the thesis can be summarized as follows.

Chapter 2: In chapter 2, the polar line-wise set of power balance equations and its solution using NR technique is developed. The formulation uses square of voltage magnitude instead of using voltage magnitudes as such. The use of square of voltage magnitude leads to linear terms for real and reactive powers, considering constant impedance type elements in certain portions of power balance equations. Coupled with this fact and the use of real and reactive line-wise power flows as variables, the set of linewise equations and its solution using NR technique is faster for all tested systems, up to two times, compared with bus-wise NR method. Numerical analysis of the Jacobians of bus-wise and line-wise NR methods show that the number of calculations to be completed by the line-wise NR method is much less, resulting in the higher speed of the line-wise NR method. Further, another significant benefit of the polar line-wise PF method is the ability to directly identify the set of critical lines that connect busses that are the most susceptible to VC. VCI terms appear directly in the Jacobian of the line-wise NR method. The method is both fast for PF analysis and for use as an online tool for voltage stability assessment. Summarizing, the benefits of the developed line-wise NR method are:

1. It is superior to the bus-wise power method as it solves faster up to 2 times.

2. It demonstrates a stable numerical performance, monotonic convergence, solution accuracy and scales well for larger systems.

3. It provides active and reactive line power flows with no further need for computation. It does not need the system admittance matrix.

4. Its system Jacobian construction is straightforward and requires no knowledge of the system topology.

5. It provides VCI values without additional computation.

6. It is very effective and time saving for voltage stability assessment analysis and ranking contingency scenarios according to their proximity to $\mathrm{VC}$.

Chapter 3: In Chapter 3, a rectangular line-wise PF is developed which uses of real and imaginary voltage terms and leads to avoiding trigonometric functions. The set of rectangular line-wise equations and its solution using NR technique is faster for all tested systems, up to three times, compared with the bus-wise NR method. Numerical analysis of the Jacobians of rectangular bus-wise and line-wise NR methods show 
that the number of calculations to be completed by the rectangular line-wise NR method is much lower, resulting in the higher speed of the rectangular line-wise NR method.

The rectangular line-wise PF method is tested and compared for performance with existing polar and rectangular bus-wise PF methods respectively on 6-, 14-, 57- and 118-bus IEEE systems, a 582-bus real power system, a 2383-bus Polish power system, and a 9241-bus PEGASE system. The results show that the rectangular form line-wise NR method demonstrates a stable numerical performance, monotonic convergence, up to thrice the speed in computation, and solution accuracy. It scales well, as shown, for larger systems. The active and reactive line power flows result directly from the solution with no further need for computation. The need to construct the system admittance matrix is eliminated. Development of the system Jacobian is straightforward and requires no knowledge of the system topology.

Chapter 4: In Chapter 4, a new formulation for optimal power flow based on a set of line-wise power flow equations is proposed. The line-wise optimal power flow (LWOPF) formulation is linearized and solved using successive linear programming technique (SLP). The proposed LWOPF-SLP method is tested on 6-, 14-, 30-, 57- and 118-bus IEEE systems, a 582-bus real power system, a 2383-bus Polish power system, and a 9241-bus PEGASE system and compared for performance with traditional bus-wise OPF (BWOPF) implemented in MATPOWER and the best known SLP implementation in the literature.

The benefits of the LWOPF method include: (1) stable numerical performance when scaled to large power systems, (2) higher speed, up to two times than that of the BWOPF method, (3) it provides a monotonic convergence, and, (4) a better optimal solution results due to improved solution space modeling using square of voltage magnitudes. The proposed LWOPF method, due to its simplicity in implementation, higher speed of execution, monotonic convergence characteristics, and, the ability to obtain a better optimal solution, is well suited for commercial power systems application.

Chapter 5: In Chapter 5, a novel simple nonlinear formulation for optimal power flow based on a set of line-wise power flow equations is proposed. The line-wise optimal power flow (LWOPF) formulation is developed and solved using the FMINCON nonlinear solver. The proposed LWOPF formulation is tested on 6-, 14-, 57- and 118-bus IEEE systems, and a 582-bus real power system and compared for performance with traditional bus-wise OPF (BWOPF) using MATPOWER.

Results of proposed LWOPF formulation demonstrate the stable numerical performance when scaled to large power systems, its higher speed, up to two times than that of the BWOPF method, and, a better 
optimal solution due to improved solution space modelling using square of voltage magnitudes. Further, the Line-wise OPF formulation is augmented, incorporating the voltage stability constraints to create a voltage stability constrained LWOPF (VSCLWOPF) method. It shows that while ensuring safe operation for power systems with better voltage stability, generation cost is reduced in comparison with the bus-wise power flow voltage stability constrained OPF formulation. The better solution space, stemming from the formulation based upon square of voltage magnitudes, yields a lower costing optimal solution for small and large sized power systems.

Chapter 6: Finally, in Chapter 6, two novel linearized models for incorporating the voltage stability with the optimal power flow based on a set of line-wise power flow equations and line voltage collapse index are developed. The line-wise optimal power flow (LWOPF) formulation is linearized and incorporated with the set of linearized voltage collapse indices in two ways; first, VCIs are considered as a set of constraints, second, the sum of VCIs is considered as an objective function. The two developed models are solved using successive linear programming technique (SLP). The two developed linear models for incorporation of voltage stability with LWOPF are tested on 6-, 14-, and 118-bus IEEE systems and compared with the solution of linearized line-wise OPF (LWOPF).

The results show the effectiveness of the developed models for enhancing the voltage stability for power systems, even under severe contingency conditions such as line outage or stressed loading. The developed models are compared with an existing voltage stability constrained optimal power flow method and show better performance in terms of minimizing the cost function and achieving a better voltage stable system. 


\subsection{Future recommendations}

Many future works could be recommended based on the developed formulations and algorithms of this thesis. To name a few, the two developed polar and rectangular line-wise PF methods could be modified to solve unbalanced three-phase distribution systems. Moreover, they can be reformulated to be employed for solution of AC/DC PF for AC/DC microgrids. The developed nonlinear and linearized LWOPF methods could be modified to be a reactive power planning problem and could be implemented in multi-objective optimization problems. Further, the developed voltage stability incorporation with LWOPF formulations could be easily combined with other stability constraints such as the transient stability or the small signal stability. 


\section{APPENDIX}

This appendix provides more details about the set of equation in (4.20) that can be written with the non-zero terms, as follows:

$$
\begin{aligned}
& \Delta \mathrm{f} g(\boldsymbol{W})=\left[\begin{array}{l}
\Delta F F \\
\Delta F S \\
\Delta F A \\
\Delta F B \\
\Delta F P \\
\Delta F Q
\end{array}\right] \\
& \Delta F F=\frac{\partial F F}{\partial U} \cdot \Delta U+\frac{\partial F F}{\partial P F} \cdot \Delta P F+\frac{\partial F F}{\partial Q F} \cdot \Delta Q F=0 \\
& \Delta F S=\frac{\partial F S}{\partial U} \cdot \Delta U+\frac{\partial F S}{\partial P S} \cdot \Delta P S+\frac{\partial F S}{\partial Q S} \cdot \Delta Q S=0 \\
& \Delta F A=\frac{\partial F A}{\partial U} \cdot \Delta U+\frac{\partial F A}{\partial \delta} \cdot \Delta \delta+\frac{\partial F A}{\partial P F} \cdot \Delta P F+\frac{\partial F A}{\partial Q F} \cdot \Delta Q F=0 \\
& \Delta F B=\frac{\partial F B}{\partial U} \cdot \Delta U+\frac{\partial F B}{\partial \delta} \cdot \Delta \delta+\frac{\partial F B}{\partial P S} \cdot \Delta P S+\frac{\partial F B}{\partial Q S} \cdot \Delta Q S=0 \\
& \Delta F P=\frac{\partial F P}{\partial U} \cdot \Delta U+\frac{\partial F P}{\partial P F} \cdot \Delta P F+\frac{\partial F P}{\partial P S} \cdot \Delta P S+\frac{\partial F P}{\partial P G} \cdot \Delta P G=0 \\
& \Delta F Q=\frac{\partial F Q}{\partial U} \cdot \Delta U+\frac{\partial F Q}{\partial Q F} \cdot \Delta Q F+\frac{\partial F Q}{\partial Q S} \cdot \Delta Q S+\frac{\partial F Q}{\partial Q G} \cdot \Delta Q G=0
\end{aligned}
$$

All the terms in the set of equations (A.2) - (A.7) are derived in the Jacobian section 2.4. The two terms $\frac{\partial F P}{\partial P G}$ and $\frac{\partial F Q}{\partial Q G}$ equals to one at generation buses, and otherwise their values are zeros. 


\section{REFERENCES}

[1] H. Smith, "Calculation of power flow and bus voltages," Electrical Engineering, vol. 54, pp. 408416, 1935.

[2] W. E. Enns, "A new simple calculator of load flow in AC networks," Transactions of the American Institute of Electrical Engineers, vol. 62, pp. 786-790, 1943.

[3] J. B. Ward, "Equivalent circuits for power-flow studies," Electrical Engineering, vol. 68, pp. 794794, 1949.

[4] L. A. Dunstan, "Digital load flow studies," Transactions of the American Institute of Electrical Engineers. Part III: Power Apparatus and Systems, vol. 73, pp. 825-832, 1954.

[5] J. Bennett, "Digital computers and the load-flow problem," Proceedings of the IEE-Part B: Radio and Electronic Engineering, vol. 103, pp. 16-25, 1956.

[6] J. Calvert and T. Sze, "Some general theorems on power flow in linear networks," Transactions of the American Institute of Electrical Engineers. Part III: Power Apparatus and Systems, vol. 75, pp. 984-988, 1956.

[7] R. Jordan, "Rapidly converging digital load flow," Transactions of the American Institute of Electrical Engineers. Part III: Power Apparatus and Systems, vol. 76, pp. 1433-1438, 1957.

[8] W. F. Tinney and C. E. Hart, "Power flow solution by Newton's method," IEEE Transactions on Power Apparatus and systems, pp. 1449-1460, 1967.

[9] B. Stott and O. Alsac, "Fast decoupled load flow," IEEE transactions on power apparatus and systems, pp. 859-869, 1974.

[10] Y. Tamura, H. Mori, and S. Iwamoto, "Relationship between voltage instability and multiple load flow solutions in electric power systems," IEEE Transactions on Power Apparatus and Systems, pp. $1115-1125,1983$.

[11] G.-X. Luo and A. Semlyen, "Efficient load flow for large weakly meshed networks," IEEE Transactions on Power Systems, vol. 5, pp. 1309-1316, 1990.

[12] F. D. Galiana and Z.-C. Zeng, "Analysis of the load flow behaviour near a Jacobian singularity," IEEE transactions on power systems, vol. 7, pp. 1362-1369, 1992.

[13] D. J. Tylavsky, P. Crouch, L. F. Jarriel, and R. Adapa, "Improved power flow robustness for personal computers," IEEE transactions on industry applications, vol. 28, pp. 1102-1108, 1992.

[14] A. Semlyen, "Fundamental concepts of a Krylov subspace power flow methodology," IEEE Transactions on Power Systems, vol. 11, pp. 1528-1537, 1996.

[15] Y. Chen and C. Shen, "A Jacobian-free Newton-GMRES (m) method with adaptive preconditioner and its application for power flow calculations," IEEE Transactions on Power Systems, vol. 21, pp. 1096-1103, 2006.

[16] K. L. Lo and C. Zhang, "Decomposed three-phase power flow solution using the sequence component frame," in IEE Proceedings C-Generation, Transmission and Distribution, pp. 181-188, 1993.

[17] X.-P. Zhang and H. Chen, "Asymmetrical three-phase load-flow study based on symmetrical component theory," IEE Proceedings-Generation, Transmission and Distribution, vol. 141, pp. 248$252,1994$.

[18] C. S. Cheng and D. Shirmohammadi, "A three-phase power flow method for real-time distribution system analysis," IEEE Transactions on Power Systems, vol. 10, pp. 671-679, 1995. 
[19] M. M. A. Abdelaziz, H. E. Farag, E. F. El-Saadany, and Y. A.-R. I. Mohamed, "A novel and generalized three-phase power flow algorithm for islanded microgrids using a newton trust region method," IEEE Transactions on Power Systems, vol. 28, pp. 190-201, 2013.

[20] M. J. Alam, K. Muttaqi, and D. Sutanto, "A three-phase power flow approach for integrated 3-wire MV and 4-wire multigrounded LV networks with rooftop solar PV," IEEE Transactions on Power Systems, vol. 28, pp. 1728-1737, 2013.

[21] I. Dzafic, H.-T. Neisius, M. Gilles, S. Henselmeyer, and V. Landerberger, "Three-phase power flow in distribution networks using Fortescue transformation," IEEE Transactions on Power Systems, vol. 28, pp. 1027-1034, 2013.

[22] N.-C. Yang, "Three-phase power flow calculations using direct Z BUS method for large-scale unbalanced distribution networks," IET Generation, Transmission \& Distribution, vol. 10, pp. 10481055, 2016.

[23] A. Garces, "A linear three-phase load flow for power distribution systems," IEEE Transactions on Power Systems, vol. 31, pp. 827-828, 2016.

[24] J. E. Van Ness and J. H. Griffin, "Elimination methods for load-flow studies," Transactions of the American Institute of Electrical Engineers. Part III: Power Apparatus and Systems, vol. 80, pp. 299302, 1961.

[25] B. Stott, "Review of load-flow calculation methods," Proceedings of the IEEE, vol. 62, pp. 916-929, 1974.

[26] S. Iwamoto and Y. Tamura, "A fast load flow method retaining nonlinearity," The transactions of the Institute of Electrical Engineers of Japan. B, vol. 98, pp. 192-198, 1978.

[27] P. N. Rao, K. S. P. Rao, and J. Nanda, "An exact fast load flow method including second order terms in rectangular coordinates," IEEE Transactions on Power Apparatus and Systems, pp. 3261-3268, 1982.

[28] A. G. Expósito and E. R. Ramos, "Augmented rectangular load flow model," IEEE Transactions on Power Systems, vol. 17, pp. 271-276, 2002.

[29] P. Kundur, N. J. Balu, and M. G. Lauby, Power system stability and control vol. 7: McGraw-hill New York, 1994.

[30] C. W. Taylor, Power system voltage stability: McGraw-Hill, 1994.

[31] B. Gao, G. Morison, and P. Kundur, "Voltage stability evaluation using modal analysis," IEEE transactions on power systems, vol. 7, pp. 1529-1542, 1992.

[32] V. Ajjarapu and C. Christy, "The continuation power flow: a tool for steady state voltage stability analysis," IEEE transactions on Power Systems, vol. 7, pp. 416-423, 1992.

[33] H. Song, S. Kim, B. Lee, S.-H. Kwon, and V. Ajjarapu, "Determination of interface flow margin using the modified continuation power flow in voltage stability analysis," IEE ProceedingsGeneration, Transmission and Distribution, vol. 148, pp. 128-132, 2001.

[34] X.-P. Zhang, P. Ju, and E. Handschin, "Continuation three-phase power flow: A tool for voltage stability analysis of unbalanced three-phase power systems," IEEE transactions on power systems, vol. 20, pp. 1320-1329, 2005.

[35] F. Milano, "Continuous Newton's Method for Power Flow Analysis," IEEE Transactions on Power Systems, vol. 24, pp. 50-57, 2009.

[36] P.-A. Lof, G. Andersson, and D. Hill, "Voltage stability indices for stressed power systems," IEEE transactions on power systems, vol. 8, pp. 326-335, 1993.

[37] T. J. Overbye and C. L. DeMarco, "Improved techniques for power system voltage stability assessment using energy methods," IEEE Transactions on Power Systems, vol. 6, pp. 1446-1452, 1991. 
[38] C. A. Canizares, "On bifurcations, voltage collapse and load modeling," IEEE transactions on power systems, vol. 10, pp. 512-522, 1995.

[39] V. Balamourougan, T. Sidhu, and M. Sachdev, "Technique for online prediction of voltage collapse," IEE Proceedings-Generation, Transmission and Distribution, vol. 151, pp. 453-460, 2004.

[40] G. Verbic and F. Gubina, "A new concept of voltage collapse protection based on apparent power losses," 2004 Eighth IEE International Conference on Developments in Power System Protection, Amsterdam, Netherlands, Vol.2, pp. 635-638, 2004.

[41] B. Venkatesh, A. Rost, and L. Chang, "Dynamic voltage collapse index-wind generator application," IEEE transactions on power delivery, vol. 22, pp. 90-94, 2007.

[42] S. G. Ghiocel and J. H. Chow, "A power flow method using a new bus type for computing steadystate voltage stability margins," IEEE Transactions on Power Systems, vol. 29, pp. 958-965, 2014.

[43] M. B. Cain, R. P. O'neill, and A. Castillo, "History of optimal power flow and formulations," Federal Energy Regulatory Commission, pp. 1-36, 2012.

[44] J. A. Momoh, M. E. El-Hawary, and R. Adapa, "A review of selected optimal power flow literature to 1993. II. Newton, linear programming and interior point methods," IEEE Transactions on Power Systems, vol. 14, pp. 105-111, 1999.

[45] J. A. Momoh, R. Adapa, and M. E. El-Hawary, "A review of selected optimal power flow literature to 1993. I. Nonlinear and quadratic programming approaches," IEEE Transactions on Power Systems, vol. 14, pp. 96-104, 1999.

[46] J. Carpentier, "Contribution a l'etude du Dispatching Economique," Bulletin de la Societe Francaise des Electriciens, vol. 3, pp. 431-447, Aug. 1962.

[47] A. H. El-abiad and F. J. Jaimes, "A method for optimum scheduling of power and voltage magnitude," IEEE Transactions on power Apparatus and systems, pp. 413-422, 1969.

[48] H. W. Dommel and W. F. Tinney, "Optimal power flow solutions," IEEE Transactions on power apparatus and systems, pp. 1866-1876, 1968.

[49] A. M. Sasson, F. Viloria, and F. Aboytes, "Optimal Load Flow Solution Using the Hessian Matrix," IEEE Transactions on Power Apparatus and Systems, vol. PAS-92, pp. 31-41, 1973.

[50] A. Rashed and D. Kelly, "Optimal load flow solution using Lagrangian multipliers and the Hessian matrix," IEEE Transactions on Power Apparatus and Systems, pp. 1292-1297, 1974.

[51] O. Alsac and B. Stott, "Optimal Load Flow with Steady-State Security," IEEE Transactions on Power Apparatus and Systems, vol. PAS-93, pp. 745-751, 1974.

[52] R. Burchett, H. Happ, and K. Wirgau, "Large scale optimal power flow," IEEE Transactions on Power Apparatus and Systems, pp. 3722-3732, 1982.

[53] R. Burchett, H. Happ, and D. Vierath, "Quadratically convergent optimal power flow," IEEE Transactions on Power Apparatus and Systems, pp. 3267-3275, 1984.

[54] K. Aoki, A. Nishikori, and R. Yokoyama, "Constrained load flow using recursive quadratic programming," IEEE transactions on power systems, vol. 2, pp. 8-16, 1987.

[55] G. Contaxis, C. Delkis, and G. Korres, "Decoupled optimal load flow using linear or quadratic programming," IEEE Transactions on Power systems, vol. 1, pp. 1-7, 1986.

[56] M. Abido, "Optimal power flow using particle swarm optimization," International Journal of Electrical Power \& Energy Systems, vol. 24, pp. 563-571, 2002.

[57] A. G. Bakirtzis, P. N. Biskas, C. E. Zoumas, and V. Petridis, "Optimal power flow by enhanced genetic algorithm," IEEE Transactions on power Systems, vol. 17, pp. 229-236, 2002.

[58] L. L. Lai, J. Ma, R. Yokoyama, and M. Zhao, "Improved genetic algorithms for optimal power flow under both normal and contingent operation states," International Journal of Electrical Power \& Energy Systems, vol. 19, pp. 287-292, 1997. 
[59] T. Sousa, J. Soares, Z. A. Vale, H. Morais, and P. Faria, "Simulated annealing metaheuristic to solve the optimal power flow," in 2011 IEEE Power and Energy Society General Meeting, pp. 1-8, 2011.

[60] T. T. Nguyen, "Neural network optimal-power-flow," in 1997 Fourth International Conference on Advances in Power System Control, Operation and Management, APSCOM-97, pp. 266-271 vol.1, 1997.

[61] R. A. Jabr, "Exploiting sparsity in SDP relaxations of the OPF problem," IEEE Transactions on Power Systems, vol. 27, pp. 1138-1139, 2012.

[62] D. K. Molzahn, B. C. Lesieutre, and C. L. DeMarco, "A sufficient condition for global optimality of solutions to the optimal power flow problem," IEEE Transactions on Power Systems, vol. 29, pp. 978-979, 2014.

[63] J. Lavaei and S. H. Low, "Zero duality gap in optimal power flow problem," IEEE Transactions on Power Systems, vol. 27, pp. 92-107, 2012.

[64] S. H. Low, "Convex relaxation of optimal power flow_Part I: Formulations and equivalence," IEEE Transactions on Control of Network Systems, vol. 1, pp. 15-27, 2014.

[65] R. Madani, S. Sojoudi, and J. Lavaei, "Convex relaxation for optimal power flow problem: Mesh networks," IEEE Transactions on Power Systems, vol. 30, pp. 199-211, 2015.

[66] Z. Qin, Y. Hou, and Y. Chen, "Convex envelopes of optimal power flow with branch flow model in rectangular form," in 2015 IEEE Power \& Energy Society General Meeting, pp. 1-5, 2015.

[67] D. K. Molzahn and I. A. Hiskens, "Convex Relaxations of Optimal Power Flow Problems: An Illustrative Example," IEEE Transactions on Circuits and Systems I: Regular Papers, vol. 63, pp. 650-660, 2016.

[68] J. A. Taylor, Convex optimization of power systems: Cambridge University Press, 2015.

[69] L. Roald, G. Andersson, S. Misra, M. Chertkov, and S. Backhaus, "Optimal power flow with wind power control and limited expected risk of overloads," in Power Systems Computation Conference (PSCC), pp. 1-7, 2016.

[70] A. Kargarian, et al., "Toward distributed/decentralized DC optimal power flow implementation in future electric power systems," IEEE Transactions on Smart Grid, vol. 9, no. 4, pp. 2574-2594, July 2018.

[71] J. Rimez, "Optimal Power Flow modeling of hybrid AC/DC systems," HVDC Grids: For Offshore and Supergrid of the Future, vol. 51, p. 293, 2016.

[72] S. Gill, I. Kockar, and G. W. Ault, "Dynamic optimal power flow for active distribution networks," IEEE Transactions on Power Systems, vol. 29, pp. 121-131, 2014.

[73] M. Madrigal and A. Quintana, "Semidefinite programming relaxations for $\{0,1\}$-power dispatch problems," in Power Engineering Society Summer Meeting, pp. 697-7021999.

[74] R. Fuentes-Loyola and V. H. Quintana, "Medium-term hydrothermal coordination by semidefinite programming," IEEE Transactions on Power Systems, vol. 18, pp. 1515-1522, 2003.

[75] R. A. Jabr, "Modeling network losses using quadratic cones," IEEE Transactions on Power Systems, vol. 20, pp. 505-506, 2005.

[76] R. A. Jabr, "Radial distribution load flow using conic programming," IEEE transactions on power systems, vol. 21, pp. 1458-1459, 2006.

[77] X. Bai, H. Wei, K. Fujisawa, and Y. Wang, "Semidefinite programming for optimal power flow problems," International Journal of Electrical Power \& Energy Systems, vol. 30, pp. 383-392, 2008.

[78] X. Bai and H. Wei, "A semidefinite programming method with graph partitioning technique for optimal power flow problems," International Journal of Electrical Power \& Energy Systems, vol. 33, pp. 1309-1314, 2011. 
[79] M. Farivar and S. H. Low, "Branch Flow Model: Relaxations and Convexification-Part II," IEEE Transactions on Power Systems, vol. 28, pp. 2565-2572, 2013.

[80] M. Farivar and S. H. Low, "Branch flow model: Relaxations and convexification-Part I," IEEE Transactions on Power Systems, vol. 28, pp. 2554-2564, 2013.

[81] S. H. Low, "Convex relaxation of optimal power flow-Part II: Exactness," IEEE Transactions on Control of Network Systems, vol. 1, pp. 177-189, 2014.

[82] L. Gan and S. H. Low, "Convex relaxations and linear approximation for optimal power flow in multiphase radial networks," in Power Systems Computation Conference (PSCC), pp. 1-9, 2014.

[83] A. Kalbat, R. Madani, G. Fazelnia, and J. Lavaei, "Efficient convex relaxation for stochastic optimal distributed control problem," in Communication, Control, and Computing (Allerton), 2014 52nd Annual Allerton Conference on, pp. 589-596, 2014.

[84] S. Y. Abdelouadoud, R. Girard, F.-P. Neirac, and T. Guiot, "Iterative linear cuts strenghtening the second-order cone relaxation of the distribution system optimal power flow problem," in $T \& D$ Conference and Exposition, 2014 IEEE PES, pp. 1-4, 2014.

[85] J. Tian, H. Wei, and J. Tan, "Global optimization for power dispatch problems based on theory of moments," International Journal of Electrical Power \& Energy Systems, vol. 71, pp. 184-194, 2015.

[86] L. Gan, N. Li, U. Topcu, and S. H. Low, "Exact convex relaxation of optimal power flow in radial networks," IEEE Transactions on Automatic Control, vol. 60, pp. 72-87, 2015.

[87] S. Bose, S. H. Low, T. Teeraratkul, and B. Hassibi, "Equivalent relaxations of optimal power flow," IEEE Transactions on Automatic Control, vol. 60, pp. 729-742, 2015.

[88] R. A. Jabr and I. Džafić, "A compensation-based conic opf for weakly meshed networks," IEEE Transactions on Power Systems, vol. 31, pp. 4167-4168, 2016.

[89] A. Hermann, Q. Wu, S. Huang, and A. H. Nielsen, "Convex relaxation of optimal power flow in distribution feeders with embedded solar power," Energy Procedia, vol. 100, pp. 43-49, 2016.

[90] S. Huang, Q. Wu, J. Wang, and H. Zhao, "A sufficient condition on convex relaxation of AC optimal power flow in distribution networks," IEEE Transactions on Power Systems, vol. 32, no. 2, pp. 13591368, March 2017.

[91] Q. Li and V. Vittal, "The convex hull of the AC power flow equations in rectangular coordinates," in 2016 IEEE Power and Energy Society General Meeting, Boston, pp. 1-5, May 2016.

[92] Q. Li and V. Vittal, "non-iterative enhanced SDP relaxations for optimal scheduling of distributed energy storage in distribution systems," in IEEE Transactions on Power Systems, vol. 32, no. 3, pp. 1721-1732, May 2017.

[93] Q. Li, R. Ayyanar, and V. Vittal, "Convex optimization for DES planning and operation in radial distribution systems with high penetration of photovoltaic resources," IEEE Transactions on Sustainable Energy, vol. 7, pp. 985-995, 2016.

[94] B. Kocuk, S. S. Dey, and X. A. Sun, "Inexactness of SDP relaxation and valid inequalities for optimal power flow," IEEE Transactions on Power Systems, vol. 31, pp. 642-651, 2016.

[95] B. Stott, J. Marinho, and O. Alsac, "Review of linear programming applied to power system rescheduling," in Power Industry Computer Applications Conference, 1979. PICA-79. IEEE Conference Proceedings, pp. 142-154, 1979.

[96] K. Iba, H. Suzuki, K.-I. Suzuki, and K. Suzuki, "Practical reactive power allocation/operation planning using successive linear programming," IEEE Transactions on Power Systems, vol. 3, pp. 558-566, 1988.

[97] B. Venkatesh, M. Abdullah Khan, "Fuzzy logic based successive LP method for reactive power optimization," Electric Machines \&Power Systems, vol. 27, pp. 1141-1160, 1999. 
[98] A. J. Urdaneta, J. F. Gomez, E. Sorrentino, L. Flores, and R. Diaz, "A hybrid genetic algorithm for optimal reactive power planning based upon successive linear programming," IEEE Transactions on power systems, vol. 14, pp. 1292-1298, 1999.

[99] B. Stott and J. Marinho, "Linear programming for power-system network security applications," IEEE Transactions on Power Apparatus and Systems, pp. 837-848, 1979.

[100] B. Stott and O. Alsac, "Experience with successive linear programming for optimal rescheduling of active and reactive power," in CIGRE-IFAC Symp. on Control Applications to Power System Security, 1983.

[101] R. Mota-Palomino and V. Quintana, "A penalty function-linear programming method for solving power system constrained economic operation problems," IEEE transactions on power apparatus and systems, pp. 1414-1422, 1984.

[102] D. Reinstein, F. Galiana, and A. Vojdani, "Probabilistic simulation of a power system under transmission constrained economic dispatch," IEEE transactions on power systems, vol. 1, pp. 248255, 1986.

[103] D. S. Kirschen and H. P. Van Meeteren, "MW/voltage control in a linear programming based optimal power flow," IEEE Transactions on Power Systems, vol. 3, pp. 481-489, 1988.

[104] L. S. Vargas, V. H. Quintana, and A. Vannelli, "A tutorial description of an interior point method and its applications to security-constrained economic dispatch," IEEE Transactions on Power Systems, vol. 8, pp. 1315-1324, 1993.

[105] G. L. Torres and V. H. Quintana, "Optimal power flow via interior point methods: an educational tool in Matlab," in Electrical and Computer Engineering, 1996. Canadian Conference, pp. 996-999, 1996.

[106] K. Zehar and S. Sayah, "Optimal power flow with environmental constraint using a fast successive linear programming algorithm: Application to the algerian power system," Energy Conversion and Management, vol. 49, pp. 3362-3366, 2008.

[107] Z. Yang, H. Zhong, Q. Xia, A. Bose, and C. Kang, "Optimal power flow based on successive linear approximation of power flow equations," IET Generation, Transmission \& Distribution, vol. 10, pp. 3654-3662, 2016.

[108] A. Castillo, P. Lipka, J.-P. Watson, S. S. Oren, and R. P. O’Neill, "A successive linear programming approach to solving the IV-ACOPF," IEEE Trans. Power Syst, vol. 31, pp. 2752-2763, 2016.

[109] C. Chompoo-Inwai, W.-J. Lee, P. Fuangfoo, M. Williams, and J. R. Liao, "System impact study for the interconnection of wind generation and utility system," IEEE transactions on Industry Applications, vol. 41, pp. 163-168, 2005.

[110] S. Eftekharnejad, V. Vittal, G. T. Heydt, B. Keel, and J. Loehr, "Impact of increased penetration of photovoltaic generation on power systems," IEEE Transactions on Power Systems, vol. 28, pp. 893901, 2013.

[111] M. J. Hossain, H. R. Pota, M. A. Mahmud, and R. A. Ramos, "Investigation of the impacts of largescale wind power penetration on the angle and voltage stability of power systems," IEEE Systems Journal, vol. 6, pp. 76-84, 2012.

[112] R. Toma and M. Gavrilas, "The impact on voltage stability of the integration of renewable energy sources into the electricity grids," in Electrical and Power Engineering (EPE), 2014 International Conference and Exposition on, pp. 1051-1054, 2014.

[113] H. Amarasekara, L. Meegahapola, A. Agalgaonkar, and S. Perera, "Impact of renewable power integration on VQ stability margin," in Power Engineering Conference (AUPEC), 2013 Australasian Universities, pp. 1-6, 2013. 
[114] A. Berizzi, C. Bovo, M. Innorta, and P. Marannino, "Multiobjective optimization techniques applied to modern power systems," in Power Engineering Society Winter Meeting, pp. 1503-1508, 2001.

[115] J. Zhao, B. Zhang, and H.-D. Chiang, "An optimal power flow model and approach with static voltage stability constraints," in Transmission and Distribution Conference and Exhibition: Asia and Pacific, 2005 IEEE/PES, pp. 1-6, 2005.

[116] G. Wu, C. Chung, K. Wong, and C. Yu, "Voltage stability constrained optimal dispatch in deregulated power systems," IET Generation, Transmission \& Distribution, vol. 1, pp. 761-768, 2007.

[117] S. Jeyadevi, S. Baskar, and M. W. Iruthayarajan, "Reactive power planning with voltage stability enhancement using covariance matrix adapted evolution strategy," International Transactions on Electrical Energy Systems, vol. 21, pp. 1343-1360, 2011.

[118] T. Zabaiou and L. A. Dessaint, "VSC-OPF based on line voltage indices for power system losses minimization and voltage stability improvement," in 2013 IEEE Power and Energy Society General Meeting , pp. 1-5, 2013.

[119] D. Romero-Romero, J. Gomez-Hernandez, and J. Robles-Garcia, "Reliability optimisation of bulk power systems including voltage stability," IEE Proceedings-Generation, Transmission and Distribution, vol. 150, pp. 561-566, 2003.

[120] Y.-L. Chen, "Weak bus oriented reactive power planning for system security," IEE ProceedingsGeneration, Transmission and Distribution, vol. 143, pp. 541-545, 1996.

[121] C. Dai, W. Chen, Y. Zhu, and X. Zhang, "Reactive power dispatch considering voltage stability with seeker optimization algorithm," Electric Power Systems Research, vol. 79, pp. 1462-1471, 2009.

[122] P. Roy, B. Mandal, and K. Bhattacharya, "Gravitational search algorithm based optimal reactive power dispatch for voltage stability enhancement," Electric Power Components and Systems, vol. 40, pp. 956-976, 2012.

[123] B. D. Thukaram and K. Parthasarathy, "Optimal reactive power dispatch algorithm for voltage stability improvement," International Journal of Electrical Power \& Energy Systems, vol. 18, pp. 461-468, 1996.

[124] T. Niknam, M. Narimani, J. Aghaei, and R. Azizipanah-Abarghooee, "Improved particle swarm optimisation for multi-objective optimal power flow considering the cost, loss, emission and voltage stability index," IET generation, transmission \& distribution, vol. 6, pp. 515-527, 2012.

[125] D. Devaraj and J. P. Roselyn, "Genetic algorithm based reactive power dispatch for voltage stability improvement," International Journal of Electrical Power \& Energy Systems, vol. 32, pp. 1151-1156, 2010.

[126] C.-F. Yang, G. G. Lai, C.-H. Lee, C.-T. Su, and G. W. Chang, "Optimal setting of reactive compensation devices with an improved voltage stability index for voltage stability enhancement," International Journal of Electrical Power \& Energy Systems, vol. 37, pp. 50-57, 2012.

[127] T. Zabaiou, L.-A. Dessaint, and I. Kamwa, "Preventive control approach for voltage stability improvement using voltage stability constrained optimal power flow based on static line voltage stability indices," IET Generation, Transmission \& Distribution, vol. 8, pp. 924-934, 2014.

[128] M. Todescato, J. W. Simpson-Porco, F. Dörfler, R. Carli, and F. Bullo, "Online distributed voltage stress minimization by optimal feedback reactive power control," IEEE Transactions on Control of Network Systems, 2017.

[129] W. Rosehart, C. Cañizares, and V. Quintana, "Optimal power flow incorporating voltage collapse constraints," in Power Engineering Society Summer Meeting, pp. 820-825, 1999. 
[130] C. Cañizares, W. Rosehart, A. Berizzi, and C. Bovo, "Comparison of voltage security constrained optimal power flow techniques," in Power Engineering Society Summer Meeting, pp. 1680-1685, 2001.

[131] F. Milano, C. A. Cañizares, and A. J. Conejo, "Sensitivity-based security-constrained OPF market clearing model," IEEE Transactions on power systems, vol. 20, pp. 2051-2060, 2005.

[132] M. Chávez-Lugo, C. R. Fuerte-Esquivel, C. A. Cañizares, and V. J. Gutierrez-Martinez, "Practical security boundary-constrained DC optimal power flow for electricity markets," IEEE Transactions on Power Systems, vol. 31, pp. 3358-3368, 2016.

[133] B. Cui and X. A. Sun, "A new voltage stability-constrained optimal power flow model: Sufficient condition, socp representation, and relaxation," in IEEE Transactions on Power Systems, vol. 33, no. 5, pp. 5092-5102, Sept. 2018.

[134] M. Baran and F. F. Wu, "Optimal sizing of capacitors placed on a radial distribution system," IEEE Transactions on power Delivery, vol. 4, pp. 735-743, 1989.

[135] R. D. Zimmerman, C. E. Murillo-Sánchez, and D. Gan, "Matpower," PSERC.[Online]. Software Available at: http://www. pserc. cornell. edu/matpower, 1997.

[136] M. K. Pal, "Voltage stability conditions considering load characteristics," IEEE transactions on power systems, vol. 7, pp. 243-249, 1992.

[137] A. A. Mohamed and B. Venkatesh, "Line-Wise power flow and voltage collapse," IEEE Transactions on Power Systems, vol. 33, , no. 4, pp. 3768-3778, July 2018.

[138] R. Fourer, D. M. Gay, and BW Kernighan," AMPL: a modeling language for mathematical programming, 1999.

[139] CPLEX Documentation: Release12.4, IBM Corp.,2014.

[140] Gurobi Documentation: Release5.6, Gurobi Optimization, Inc.,2014.

[141] C. Concordia and S. Thara, "Load representation in power system stability studies," IEEE transactions on power apparatus and systems, pp. 969-977, 1982.

[142] 2017 Yearbook of Electricity Distributors, https://www.oeb.ca/oeb/_Documents/RRR/2017_Yearbook_of_Electricity_Distributors.pdf. 